\title{
FROM THE BOLTZMANN EQUATION TO AN INCOMPRESSIBLE NAVIER-STOKES-FOURIER SYSTEM
}

\author{
C. DAVID LEVERMORE AND NADER MASMOUDI
}

\begin{abstract}
We establish a Navier-Stokes-Fourier limit for solutions of the Boltzmann equation considered over any periodic spatial domain of dimension two or more. We do this for a broad class of collision kernels that relaxes the Grad small deflection cutoff condition for hard potentials and includes for the first time the case of soft potentials. Appropriately scaled families of DiPernaLions renormalized solutions are shown to have fluctuations that are compact. Every limit point is governed by a weak solution of a Navier-Stokes-Fourier system for all time.
\end{abstract}

\section{INTRODUCTION}

We establish a Navier-Stokes-Fourier fluid dynamical limit for the classical Boltzmann equation considered over any periodic spatial domain of dimension two or more. Here the Navier-Stokes-Fourier system governs $(\rho, u, \theta)$, the fluctuations of mass density, bulk velocity, and temperature about their spatially homogeneous equilibrium values in a Boussinesq regime. Specifically, after a suitable choice of units, these fluctuations satisfy the incompressibility and Boussinesq relations

$$
\nabla_{x} \cdot u=0, \quad \rho+\theta=0 ;
$$

while their evolution is determined by the motion and heat equations

$$
\begin{aligned}
\partial_{t} u+u \cdot \nabla_{x} u+\nabla_{x} p & =\nu \Delta_{x} u, & & u(x, 0)=u^{i n}(x), \\
\frac{D+2}{2}\left(\partial_{t} \theta+u \cdot \nabla_{x} \theta\right) & =\kappa \Delta_{x} \theta, & & \theta(x, 0)=\theta^{i n}(x),
\end{aligned}
$$

where $\nu>0$ is the kinematic viscosity and $\kappa>0$ is the thermal conductivity.

This work completes the program laid out in $[2,3]$. One of the central goals of that program is to connect the DiPerna-Lions theory of global renormalized solutions of the Boltzmann equation to the Leray

Date: March 4, 2008.

to be submitted to ARMA. . 
theory of global weak solutions of the incompressible Navier-StokesFourier system (1.1-1.2). The main result of [3] for the Navier-Stokes limit is to recover the motion equation for a discrete-time version of the Boltzmann equation assuming the DiPerna-Lions solutions satisfy the local conservation of momentum and with the aid of a mild compactness assumption. This result falls short of the goal in a number of aspects. First, the heat equation was not treated because the heat flux terms could not be controlled. Second, local momentum conservation was assumed because DiPerna-Lions solutions are not known to satisfy the local conservation law of momentum (or energy) that one would formally expect. Third, the discrete-time case was treated in order to avoid having to control the time regularity of the acoustic modes. Fourth, unnatural technical assumptions were made on the Boltzmann collision kernel. Finally, a weak compactness assumption was required to pass to the limit in certain nonlinear terms. The present work removes all of these shortcomings. It builds upon the recent advances found in [36], [19] and [24].

In [36] Lions and Masmoudi recover the Navier-Stokes motion equation with the aid of only the local conservation of momentum assumption and the nonlinear weak compactness assumption that where made in [3]. However, they do not recover the heat equation and they retain the same unnatural technical assumptions made in [3] on the collision kernel. There were two key new ingredients in their work. First, they were able to control the time regularity of the acoustic modes by adapting an idea from [34]. Second, they were able to prove that the contribution of the acoustic modes to the limiting motion equation is just an extra gradient term that can be incorporated into the pressure term. There are two reasons they do not recover the heat equation. First, it is unknown whether or not DiPerna-Lions solutions satisfy local energy conservation law. Second, even if local energy conservation were assumed, the techniques they used to control the momentum flux would fail to control the heat flux.

In [19] Golse and Levermore recover the Stokes-Fourier system (the linearization of (1.1-1.2) about zero). There were two key new ingredients in their work. First, they control the local momentum and energy conservation defects of the DiPerna-Lions solutions with dissipation rate estimates that allowed them to recover these local conservation laws in the limit. Second, they also control the heat flux with dissipation rate estimates. Because they treat the linear Stokes-Fourier case in [19], they do not face the need either to control the acoustic modes or for a compactness assumption, both of which are used to pass to the limit in the nonlinear terms in [36]. 
In the present work we recover the Navier-Stokes-Fourier system. Our result goes beyond the results mentioned above by combining the new ingredients from [36] and [19] with some new nonlinear estimates and the new averaging lemma of [24]. More specifically, here we adapt the control of the acoustic modes found in [36] and the dissipation rate controls of both the heat flux and the conservation defects found in [19]. Our new estimates allow us to treat a broader class of collision kernels than was treated in either [36] or [19]. In particular, we treat all hard potential cases and, for the first time in this program, soft potential cases.

Here we do not use the fact proved in [37] that DiPerna-Lions renormalized solutions satisfy the formally expected local momentum conservation law up to the divergence of a nonnegative definite, matrixvalued defect measure that is controlled by an entropy bound. There are two reasons for this. First, unlike for the Stokes limit case treated in [37], here the entropy bound does not give enough control of the defect measure to allow us to conclude that it does not change the limiting motion equation. Second, it is unknown whether or not DiPerna-Lions solutions satisfy local energy conservation law up to the divergence of a defect measure, or how to control such a measure should it exist. In order to recover the heat equation we therefore develop estimates that control the local energy conservation defects of DiPerna-Lions solutions in the limit. These estimates do the same for the motion equation. This approach enables us to recover both the heat and motion equation without imposing any regularity requirements on the limiting Navier-Stokes-Fourier solution.

At the same time as this work was being carried out, Golse and Saint-Raymond [23, 25] were able to recover the Navier-Stokes-Fourier system without making any nonlinear weak compactness hypothesis. In addition to building on the ideas in [36] and [19], their proof uses the entropy dissipation rate to decompose the collision operator in a new way and uses a new $L^{1}$ averaging lemma [24] (which has its origins in $[41,42])$ to prove the compactness assumption. Their result in $[23$, $25]$ is restricted to a narrow class of bounded Boltzmann kernels that only includes the special case of Maxwell molecules with a Grad small deflection cut-off from among all kernels that are classically derived from an inter-particle potential. They have recently extended their result to the case of hard spheres. The present work is complementary in that it introduces estimates that apply to a broad class of cut-off kernels and especially to soft potentials.

The next section and section 4 contain preliminary material regarding the Boltzmann equation, including the formal scaling that leads 
from the Boltzmann equation to the Navier-Stokes-Fourier system as well as the formal derivation. Section 3 contains some technical assumptions about the collision kernel we consider. These assumptions are satisfied by all classical collision kernels with cutoff. Section 5 reviews the DiPerna-Lions theory of global solutions for the Boltzmann equation [15] and the Leray theory of global solutions for the NavierStokes-Fourier system. Section 6 presents precise statements of our results and section 7 contains the proof of the main results modulo four estimates: one that removes the local conservation defects, one that controls the fluxes, one that controls the spatial regularity of the fluctuations, and one that controls the time regularity of the acoustic modes. Sections 8 through 13 establish these four estimates, thereby completing our proof. 


\section{Boltzmann Equation Preliminaries}

Our starting point is the Boltzmann equation. In this section we collect the basic facts we will need about it. These will include its nondimensionalization and its formal conservation and dissipation laws.

2.1. The Boltzmann Equation. Here we will introduce the Boltzmann equation only so far as to set our notation, which is essentially that of [3]. More complete introductions to the Boltzmann equation can be found in $[11,12,17,18]$.

The state of a fluid composed of identical point particles confined to a spatial domain $\Omega \subset \mathbb{R}^{D}$ is described at the kinetic level by a mass density $F$ over the single-particle phase space $\mathbb{R}^{D} \times \Omega$. More specifically, $F(v, x, t) \mathrm{d} v \mathrm{~d} x$ gives the mass of the particles that occupy any infinitesimal volume $\mathrm{d} v \mathrm{~d} x$ centered at the point $(v, x) \in \mathbb{R}^{D} \times \Omega$ at the instant of time $t \geq 0$. To remove complications due to boundaries, we take $\Omega$ to be the periodic domain $\mathbb{T}^{D}=\mathbb{R}^{D} / \mathbb{L}^{D}$, where $\mathbb{L}^{D} \subset \mathbb{R}^{D}$ is any $D$-dimensional lattice.

If the particles interact only through a repulsive conservative interparticle force with finite range, then at low enough densities this range will be much smaller than the inter-particle spacing. In that regime all but binary collisions can be neglected when $D \geq 2$, and the evolution of $F=F(v, x, t)$ is governed by the classical Boltzmann equation [12]:

$$
\partial_{t} F+v \cdot \nabla_{x} F=\mathcal{B}(F, F), \quad F(v, x, 0)=F^{i n}(v, x) \geq 0 .
$$

The Boltzmann collision operator $\mathcal{B}$ acts only on the $v$ argument of $F$. It is formally given by

$$
\mathcal{B}(F, F)=\iint_{\mathbb{S}^{D-1} \times \mathbb{R}^{D}}\left(F_{1}^{\prime} F^{\prime}-F_{1} F\right) b\left(\omega, v_{1}-v\right) \mathrm{d} \omega \mathrm{d} v_{1},
$$

where $v_{1}$ ranges over $\mathbb{R}^{D}$ endowed with its Lebesgue measure $\mathrm{d} v_{1}$ while $\omega$ ranges over the unit sphere $\mathbb{S}^{D-1}=\left\{\omega \in \mathbb{R}^{D}:|\omega|=1\right\}$ endowed with its rotationally invariant measure $d \omega$. The $F_{1}^{\prime}, F^{\prime}, F_{1}$, and $F$ appearing in the integrand designate $F(\cdot, x, t)$ evaluated at the velocities $v_{1}^{\prime}, v^{\prime}, v_{1}$, and $v$ respectively, where the primed velocities are defined by

$$
v_{1}^{\prime}=v_{1}-\omega \omega \cdot\left(v_{1}-v\right), \quad v^{\prime}=v+\omega \omega \cdot\left(v_{1}-v\right),
$$

for any given $\left(\omega, v_{1}, v\right) \in \mathbb{S}^{D-1} \times \mathbb{R}^{D} \times \mathbb{R}^{D}$. Quadratic operators like $\mathcal{B}$ are extended by polarization to be bilinear and symmetric.

The unprimed and primed velocities are possible velocities for a pair of particles either before and after, or after and before, they interact 
through an elastic binary collision. Conservation of momentum and energy for particle pairs during collisions is expressed as

$$
v+v_{1}=v^{\prime}+v_{1}^{\prime}, \quad|v|^{2}+\left|v_{1}\right|^{2}=\left|v^{\prime}\right|^{2}+\left|v_{1}^{\prime}\right|^{2} .
$$

Equation (2.3) represents the general nontrivial solution of these $D+1$ equations for the $4 D$ unknowns $v_{1}^{\prime}, v^{\prime}, v_{1}$, and $v$ in terms of the $3 D-1$ parameters $\left(\omega, v_{1}, v\right)$.

The collision kernel $b$ is positive almost everywhere. The Galilean invariance of the collisional physics implies that $b$ has the classical form

$$
b\left(\omega, v_{1}-v\right)=\left|v_{1}-v\right| \Sigma\left(|\omega \cdot n|,\left|v_{1}-v\right|\right),
$$

where $n=\left(v_{1}-v\right) /\left|v_{1}-v\right|$ and $\Sigma$ is the specific differential cross-section. Technical conditions on $b$ will be imposed in Section 3 .

2.2. Nondimensionalized Form. We will work with the nondimensionalized form of the Boltzmann equation that was used in [3]. That form is motivated by the fact that the Navier-Stokes-Fourier system can be formally derived from the Boltzmann equation through a scaling in which the density $F$ is close to a spatially homogeneous Maxwellian $M=M(v)$ that has the same total mass, momentum, and energy as the initial data $F^{i n}$. By an appropriate choice of a Galilean frame and of mass, temperature and velocity units, it can be assumed that this so-called absolute Maxwellian $M$ has the form

$$
M(v) \equiv \frac{1}{(2 \pi)^{D / 2}} \exp \left(-\frac{1}{2}|v|^{2}\right) .
$$

This corresponds to the spatially homogeneous fluid state with its density and temperature equal to 1 and bulk velocity equal to 0 . This state is consistent with the form of the Navier-Stokes-Fourier system given by (1.1-1.2).

It is natural to introduce the relative kinetic density, $G=G(v, x, t)$, defined by $F=M G$. Recasting the initial-value problem (2.1) for $G$ yields

$$
\epsilon \partial_{t} G+v \cdot \nabla_{x} G=\frac{1}{\epsilon} \mathcal{Q}(G, G), \quad G(v, x, 0)=G^{i n}(v, x) .
$$

The positive, nondimensional parameter $\epsilon$ is the Knudsen number, which is the ratio of the mean-free-path to the macroscopic length scale determined by setting the volume of $\mathbb{T}^{D}$ to unity [3]. The collision operator is now given by

$$
\mathcal{Q}(G, G)=\iint_{\mathbb{S}^{D-1} \times \mathbb{R}^{D}}\left(G_{1}^{\prime} G^{\prime}-G_{1} G\right) b\left(\omega, v_{1}-v\right) \mathrm{d} \omega M_{1} \mathrm{~d} v_{1} .
$$


Under the assumptions we will make in Section 3, the nondimensional collision kernel $b$ can be normalized so that

$$
\iiint_{\mathbb{S}^{D-1} \times \mathbb{R}^{D \times \mathbb{R}^{D}}} b\left(\omega, v_{1}-v\right) \mathrm{d} \omega M_{1} \mathrm{~d} v_{1} M \mathrm{~d} v=1 .
$$

Fluid dynamical regimes are those where the mean free path is small compared to the macroscopic length scales - i.e. where the Knudsen number $\epsilon$ is small. The long-time scaling in (2.7) is consistent with a formal derivation of either the Stokes-Fourier or Navier-Stokes-Fourier systems [2].

This nondimensionalization has the normalizations

$$
\int_{\mathbb{R}^{D}} M \mathrm{~d} v=1, \quad \int_{\mathbb{T}^{D}} \mathrm{~d} x=1,
$$

associated with the domains $\mathbb{R}^{D}$ and $\mathbb{T}^{D}$ respectively, (2.9) associated with the collision kernel $b$, and

$$
\begin{gathered}
\iint_{\mathbb{R}^{D \times \mathbb{T}^{D}}} G^{i n} M \mathrm{~d} v \mathrm{~d} x=1, \quad \iint_{\mathbb{R}^{D \times \mathbb{T}^{D}}} v G^{i n} M \mathrm{~d} v \mathrm{~d} x=0, \\
\iint_{\mathbb{R}^{D \times \mathbb{T}^{D}}} \frac{1}{2}|v|^{2} G^{i n} M \mathrm{~d} v \mathrm{~d} x=\frac{D}{2} .
\end{gathered}
$$

associated with the initial data $G^{i n}$.

Because $M \mathrm{~d} v$ is a positive unit measure on $\mathbb{R}^{D}$, we denote by $\langle\xi\rangle$ the average over this measure of any integrable function $\xi=\xi(v)$ :

$$
\langle\xi\rangle=\int_{\mathbb{R}^{D}} \xi(v) M \mathrm{~d} v .
$$

Because $\mathrm{d} \mu=b\left(\omega, v_{1}-v\right) \mathrm{d} \omega M_{1} \mathrm{~d} v_{1} M \mathrm{~d} v$ is a positive unit measure on $\mathbb{S}^{D-1} \times \mathbb{R}^{D} \times \mathbb{R}^{D}$, we denote by $\langle\Xi\rangle$ the average over this measure of any integrable function $\Xi=\Xi\left(\omega, v_{1}, v\right)$ :

$$
\langle\Xi\rangle=\iiint_{\mathbb{S}^{D-1} \times \mathbb{R}^{D} \times \mathbb{R}^{D}} \Xi\left(\omega, v_{1}, v\right) \mathrm{d} \mu .
$$

The collisional measure $\mathrm{d} \mu$ is invariant under the transformations

$$
\left(\omega, v_{1}, v\right) \mapsto\left(\omega, v, v_{1}\right), \quad\left(\omega, v_{1}, v\right) \mapsto\left(\omega, v_{1}^{\prime}, v^{\prime}\right) .
$$

These, and compositions of these, are called collisional symmetries.

2.3. Formal Conservation and Dissipation Laws. We now list for later reference the basic conservation and entropy dissipation laws that are formally satisfied by solutions of the Boltzmann equation. Derivations of these laws in this nondimensional setting are outlined in [3], and can essentially be found in [11] (Sec. II.6-7), [17] (Sec. 1.4), or $[18]$. 
First, if $G$ solves the Boltzmann equation (2.7) then $G$ satisfies local conservation laws of mass, momentum, and energy:

$$
\begin{aligned}
\epsilon \partial_{t}\langle G\rangle+\nabla_{x} \cdot\langle v G\rangle & =0, \\
\epsilon \partial_{t}\langle v G\rangle+\nabla_{x} \cdot\langle v \otimes v G\rangle & =0, \\
\epsilon \partial_{t}\left\langle\frac{1}{2}|v|^{2} G\right\rangle+\nabla_{x} \cdot\left\langle v \frac{1}{2}|v|^{2} G\right\rangle & =0 .
\end{aligned}
$$

Integrating these over space and time while recalling the normalizations (2.11) of $G^{i n}$ yields the global conservation laws of mass, momentum, and energy:

$$
\begin{gathered}
\int_{\mathbb{T}^{D}}\langle G(t)\rangle \mathrm{d} x=\int_{\mathbb{T}^{D}}\left\langle G^{i n}\right\rangle \mathrm{d} x=1, \\
\int_{\mathbb{T}^{D}}\langle v G(t)\rangle \mathrm{d} x=\int_{\mathbb{T}^{D}}\left\langle v G^{i n}\right\rangle \mathrm{d} x=0, \\
\int_{\mathbb{T}^{D}}\left\langle\frac{1}{2}|v|^{2} G(t)\right\rangle \mathrm{d} x=\int_{\mathbb{T}^{D}}\left\langle\frac{1}{2}|v|^{2} G^{i n}\right\rangle \mathrm{d} x=\frac{D}{2} .
\end{gathered}
$$

Second, if $G$ solves the Boltzmann equation (2.7) then $G$ satisfies the local entropy dissipation law

$$
\begin{aligned}
\epsilon \partial_{t}\langle(G \log (G) & -G+1)\rangle+\nabla_{x} \cdot\langle v(G \log (G)-G+1)\rangle \\
& =-\frac{1}{\epsilon}\left\langle\left\langle\frac{1}{4} \log \left(\frac{G_{1}^{\prime} G^{\prime}}{G_{1} G}\right)\left(G_{1}^{\prime} G^{\prime}-G_{1} G\right)\right\rangle\right\rangle \leq 0 .
\end{aligned}
$$

Integrating this over space and time gives the global entropy equality

$$
H(G(t))+\frac{1}{\epsilon^{2}} \int_{0}^{t} R(G(s)) \mathrm{d} s=H\left(G^{i n}\right),
$$

where $H(G)$ is the relative entropy functional

$$
H(G)=\int_{\mathbb{T}^{D}}\langle(G \log (G)-G+1)\rangle \mathrm{d} x,
$$

and $R(G)$ is the entropy dissipation rate functional

$$
R(G)=\int_{\mathbb{T}^{D}}\left\langle\left\langle\frac{1}{4} \log \left(\frac{G_{1}^{\prime} G^{\prime}}{G_{1} G}\right)\left(G_{1}^{\prime} G^{\prime}-G_{1} G\right)\right\rangle \mathrm{d} x .\right.
$$




\section{Technical Assumptions on the Collision Kernel}

In this section we state all the additional assumptions that we make about the collision kernel $b$. These assumptions are satisfied by many classical collision kernels. For example, they are satisfied by the collision kernel for hard spheres of mass $m$ and radius $r_{o}$, which has the form

$$
b\left(\omega, v_{1}-v\right)=\left|\omega \cdot\left(v_{1}-v\right)\right| \frac{\left(2 r_{o}\right)^{D-1}}{2 m} .
$$

They are also satisfied by all the classical collision kernels with a small deflection cutoff that derive from a repulsive intermolecular potential of the form $c / r^{k}$ with $k>2 \frac{D-1}{D+1}$. Specifically, these have the form

$$
b\left(\omega, v_{1}-v\right)=\hat{b}(\omega \cdot n)\left|v_{1}-v\right|^{\beta} \quad \text { with } \beta=1-2 \frac{D-1}{k},
$$

where $\hat{b}(\omega \cdot n)$ is positive almost everywhere, has even symmetry in $\omega$, and satisfies the small deflection cutoff condition

$$
\int_{\mathbb{S}^{D-1}} \hat{b}(\omega \cdot n) \mathrm{d} \omega<\infty
$$

The condition $k>2 \frac{D-1}{D+1}$ is equivalent to $\beta>-D$, which insures that $b\left(\omega, v_{1}-v\right)$ is locally integrable with respect to $v_{1}-v$. The cases $\beta>0, \beta=0$, and $\beta<0$ correspond respectively to the so-called "hard", "Maxwell", and "soft" potential cases.

The works of Golse and Saint-Raymond cover the case of Maxwell potentials with a Grad small deflection cutoff [25], and the case of hard spheres [26]. Their machinery allows the treatment of the case of all hard potentials with a Grad small deflection cutoff. The Grad small deflection cutoff is much more restrictive than the cutoff (3.3) which merely guarantees the local integrability of $b$. Our work therefore relaxes their small deflection cutoff condition for hard potentials and treats for the first time the case of soft potentials.

We have already stated that the collision kernel $b$ is positive almost everywhere and has the form (2.5) - assumptions clearly met by the hard sphere and inverse power kernels given by (3.1) and (3.2). Our additional assumptions on $b$ are technical in nature - that is, they are required by our mathematical argument. We therefore examine which commonly studied physical collision kernels satisfy these assumptions. We also give some consequences of these assumptions that will play an important role in what follows. 
3.1. DiPerna-Lions Assumption. Our first assumption is that the collision kernel $b$ satisfies the requirements of the DiPerna-Lions theory. That theory requires that $b$ be locally integrable with respect to $\mathrm{d} \omega M_{1} \mathrm{~d} v_{1} M \mathrm{~d} v$, and that it moreover satisfies

$$
\begin{aligned}
& \lim _{|v| \rightarrow \infty} \frac{1}{1+|v|^{2}} \int_{K} \bar{b}\left(v_{1}-v\right) \mathrm{d} v_{1}=0, \\
& \quad \text { for every compact } K \subset \mathbb{R}^{D},
\end{aligned}
$$

where $\bar{b}$ is defined by

$$
\bar{b}\left(v_{1}-v\right) \equiv \int_{\mathbb{S}^{D-1}} b\left(\omega, v_{1}-v\right) \mathrm{d} \omega .
$$

Galilean symmetry (2.5) implies that $\bar{b}$ is a function of $\left|v_{1}-v\right|$ only.

Condition (3.4) is met by the hard sphere kernel (3.1) because in that case $\bar{b}\left(v_{1}-v\right)$ is proportional to $\left|v_{1}-v\right|$ and therefore grows like $|v|$ as $|v| \rightarrow \infty$. It is also met by the inverse power kernels (3.2) because in that case $\bar{b}\left(v_{1}-v\right)$ grows like $|v|^{\beta}$ as $|v| \rightarrow \infty$.

It is an immediate consequence of the DiPerna-Lions assumption that the measure $b\left(\omega, v_{1}-v\right) \mathrm{d} \omega M_{1} \mathrm{~d} v_{1} M \mathrm{~d} v$ is finite. The nondimensional kernel $b$ can therefore be chosen to satisfy the normalization (2.9).

3.2. Attenuation Assumption. A major role in what follows will be played by the attenuation coefficient $a$, which is defined by

$$
a(v) \equiv \int_{\mathbb{R}^{D}} \bar{b}\left(v_{1}-v\right) M_{1} \mathrm{~d} v_{1}=\iint_{\mathbb{S}^{D-1} \times \mathbb{R}^{D}} b\left(\omega, v_{1}-v\right) \mathrm{d} \omega M_{1} \mathrm{~d} v_{1} .
$$

A few facts about $a$ are readily evident from what we have already assumed. First, a must be positive and locally integrable. Because (3.4) holds, one can show that

$$
\lim _{|v| \rightarrow \infty} \frac{a(v)}{1+|v|^{2}}=0 .
$$

Next, the normalization (2.9) implies that $a$ satisfies

$$
\int_{\mathbb{R}^{D}} a M \mathrm{~d} v=1
$$

Finally, Galilean symmetry (2.5) implies that $a$ is a function of $|v|$ only.

Our second assumption regarding the collision kernel $b$ is that $a$ satisfies a lower bound of the form

$$
C_{a}(1+|v|)^{\alpha} \leq a(v)
$$

for some constants $C_{a}>0$ and $\alpha \in \mathbb{R}$. 
Condition (3.9) is met by the hard sphere kernel (3.1), for which (3.9) is satisfied with $\alpha=1$, and by all the inverse power kernels (3.2), for which (3.9) is satisfied with $\alpha=\beta$.

An immediate consequence of the attenuation assumption (3.9) is that $\frac{1}{a} \xi \in L^{p}(a M \mathrm{~d} v)$ for every $p \in[1, \infty)$ whenever $|\xi(v)|$ is bounded above by a polynomial in $|v|$.

3.3. Loss Operator Assumption. Another major role in what follows will be played by the linearized collision operator $\mathcal{L}$, which is defined formally by

$$
\mathcal{L} \tilde{g} \equiv-2 \mathcal{Q}(1, \tilde{g})=\iint_{\mathbb{S}^{D-1} \times \mathbb{R}^{D}}\left(\tilde{g}+\tilde{g}_{1}-\tilde{g}^{\prime}-\tilde{g}_{1}^{\prime}\right) b \mathrm{~d} \omega M_{1} \mathrm{~d} v_{1} .
$$

One has the decomposition

$$
\frac{1}{a} \mathcal{L}=\mathcal{I}+\mathcal{K}^{-}-2 \mathcal{K}^{+},
$$

where the integral operators $\mathcal{K}^{-}$and $\mathcal{K}^{+}$are formally defined by

$$
\begin{aligned}
\mathcal{K}^{-} \tilde{g} & \equiv \frac{1}{a} \int_{\mathbb{R}^{D}} \tilde{g}_{1} \bar{b}\left(v_{1}-v\right) M_{1} \mathrm{~d} v_{1}, \\
\mathcal{K}^{+} \tilde{g} & \equiv \frac{1}{2 a} \iint_{\mathbb{S}^{D-1} \times \mathbb{R}^{D}}\left(\tilde{g}^{\prime}+\tilde{g}_{1}^{\prime}\right) b\left(\omega, v_{1}-v\right) \mathrm{d} \omega M_{1} \mathrm{~d} v_{1} .
\end{aligned}
$$

We refer to $\mathcal{K}^{-}$as the loss operator and $\mathcal{K}^{+}$as the gain operator. For every $p \in[1, \infty]$ it can be easily shown that

$$
\left\{\begin{array}{l}
\mathcal{K}^{ \pm}: L^{p}(a M \mathrm{~d} v) \rightarrow L^{p}(a M \mathrm{~d} v) \\
\text { are bounded with }\left\|\mathcal{K}^{ \pm}\right\| \leq 1 .
\end{array}\right.
$$

The operator $\frac{1}{a} \mathcal{L}$ is therefore bounded from $L^{p}(a M \mathrm{~d} v)$ into itself for every $p \in[1, \infty]$ with $\left\|\frac{1}{a} \mathcal{L}\right\| \leq 4$.

Our third assumption regarding the collision kernel $b$ is that there exists $s \in(1, \infty]$ and $C_{b} \in(0, \infty)$ such that

$$
\left(\int_{\mathbb{R}^{D}}\left|\frac{\bar{b}\left(v_{1}-v\right)}{a\left(v_{1}\right) a(v)}\right|^{s} a\left(v_{1}\right) M_{1} \mathrm{~d} v_{1}\right)^{\frac{1}{s}} \leq C_{b} .
$$

We remark that this bound is independent of $v$. We may therefore take $C_{b}$ to be the supremum over $v$ of the left-hand side of (3.15).

Condition (3.15) is met by the hard sphere kernel (3.1) and by the inverse power kernels (3.2). We remark that for hard and Maxwell potentials $(2(D-1) \leq k)$ condition $(3.15)$ is satisfied with $s=\infty$, 
taking the form

$$
\frac{\bar{b}\left(v_{1}-v\right)}{a\left(v_{1}\right) a(v)} \leq C_{b} .
$$

This is very similar to the bound assumed in [19]. For soft potentials $\left(2 \frac{D-1}{D+1}<k<2(D-1)\right)$ condition (3.15) is satisfied for every $s$ in the interval $1<s<D /\left(\frac{2(D-1)}{k}-1\right)$. The case $s \in(1, \infty)$ in $(3.15)$ therefore allows these soft potentials to be considered.

Our third assumption (3.15) has several immediate implications for the loss operator $\mathcal{K}^{-}$, which we now express formally as

$$
\mathcal{K}^{-} \tilde{g}=\int_{\mathbb{R}^{D}} K^{-}\left(v_{1}, v\right) \tilde{g}_{1} a_{1} M_{1} \mathrm{~d} v_{1}
$$

where the kernel $K^{-}$is given by

$$
K^{-}\left(v_{1}, v\right)=\frac{\bar{b}\left(v_{1}-v\right)}{a\left(v_{1}\right) a(v)} .
$$

It is clear that $K^{-}$is symmetric $\left(K^{-}\left(v_{1}, v\right)=K^{-}\left(v, v_{1}\right)\right)$ and positive almost everywhere. An interpolation argument shows that whenever there exist $p, q, r, t \in[1, \infty]$ such that $r \leq t$ and

$$
\begin{aligned}
C_{r t} \equiv & \left(\int_{\mathbb{R}^{D}}\left(\int_{\mathbb{R}^{D}}\left|K^{-}\left(v_{1}, v\right)\right|^{r} a_{1} M_{1} \mathrm{~d} v_{1}\right)^{\frac{t}{r}} a M \mathrm{~d} v\right)^{\frac{1}{t}}<\infty, \\
& \frac{1}{p}+\frac{1}{r}+\frac{1}{t}=1+\frac{1}{q}, \quad p^{*}, q \in[r, t]
\end{aligned}
$$

then

$$
\left\{\begin{array}{l}
\mathcal{K}^{-}: L^{p}(a M \mathrm{~d} v) \rightarrow L^{q}(a M \mathrm{~d} v) \\
\text { is bounded with }\left\|\mathcal{K}^{-}\right\| \leq C_{r t} .
\end{array}\right.
$$

Moreover, whenever $r$ and $t$ in (3.19) are both finite then

$$
\mathcal{K}^{-}: L^{p}(a M \mathrm{~d} v) \rightarrow L^{q}(a M \mathrm{~d} v) \quad \text { is compact } .
$$

Here $\frac{1}{p}+\frac{1}{p^{*}}=1$. Notice that assertion (3.22) follows from assertion (3.21) because when $[r, t] \subset[1, \infty)$ the expression for $C_{r t}$ given in (3.19) defines the norm for a Banach space of kernels in which finite-rank kernels are dense.

Because $a M \mathrm{~d} v$ is a unit measure, the assumed bound (3.15) clearly implies that (3.19) holds with $C_{r t} \leq C_{b}$ for every $r \in[1, s]$ and $t \in$ 
$[1, \infty]$. Three immediate consequences of this are

$$
\begin{aligned}
& \left\{\begin{array}{l}
\mathcal{K}^{-}: L^{p}(a M \mathrm{~d} v) \rightarrow L^{p}(a M \mathrm{~d} v) \\
\text { is compact for every } p \in(1, \infty),
\end{array}\right. \\
& \left\{\begin{array}{l}
\mathcal{K}^{-}: L^{p}(a M \mathrm{~d} v) \rightarrow L^{p^{*}}(a M \mathrm{~d} v) \\
\text { is bounded with }\left\|\mathcal{K}^{-}\right\| \leq C_{b} \text { for every } p^{*} \in[1,2 s],
\end{array}\right. \\
& \left\{\begin{array}{l}
\mathcal{K}^{-}: L^{p}(a M \mathrm{~d} v) \rightarrow L^{p^{*}}(a M \mathrm{~d} v) \\
\text { is compact for every } p^{*} \in[1,2 s) .
\end{array}\right.
\end{aligned}
$$

The first of these follows from (3.22) by setting $q=p$ in (3.20) and choosing $r \in(1, s]$ such that $p, p^{*} \in\left[r, r^{*}\right]$ and then setting $t=r^{*}$. The second follows from (3.21) by setting $q=p^{*}$ in (3.20) and observing that as $r$ ranges over $[1, s]$ while $t$ ranges over $[1, \infty]$ then $p^{*}$ given by $\frac{1}{p^{*}}=\frac{1}{2}\left(\frac{1}{r}+\frac{1}{t}\right)$ will range over $[1,2 s]$. The last follows from (3.22) by excluding the cases $r=\infty$ and $t=\infty$ from the preceding consideration.

3.4. Gain Operator Assumption. Our fourth assumption regarding the collision kernel $b$ is that

$$
\mathcal{K}^{+}: L^{2}(a M \mathrm{~d} v) \rightarrow L^{2}(a M \mathrm{~d} v) \quad \text { is compact . }
$$

This condition is met by the hard sphere kernel (3.1) and by the cutoff inverse power kernels (3.2) that derive from a repulsive intermolecular potential of the form $c / r^{k}$. For $D=3$ this fact was demonstrated by Hilbert [28] for hard spheres, by Grad [27] for hard potentials $(k \geq 4)$ with a Grad small deflection cutoff, and by Golse and Poupaud [22] for soft potentials with $k>2$ with a Grad small deflection cutoff. For general $D$ this fact has recently been demonstrated by Sun [43] for kernels (3.2) that satisfy the small deflection cutoff (3.3). Even when $D=3$ this extends the result of Golse and Poupaud for soft potentials with Grad cutoffs to $k>1$.

An immediate consequence of our fourth assumption (3.26) on the gain operator $\mathcal{K}^{+}$is that

$$
\left\{\begin{array}{l}
\mathcal{K}^{+}: L^{p}(a M \mathrm{~d} v) \rightarrow L^{p}(a M \mathrm{~d} v) \\
\text { is compact for every } p \in(1, \infty) ;
\end{array}\right.
$$

This assertion follows from (3.14) and (3.26) by interpolation.

When our gain operator assumption (3.26) is combined with our loss operator assumption (3.15), we conclude that

$$
\left\{\begin{array}{l}
\frac{1}{a} \mathcal{L}: L^{p}(a M \mathrm{~d} v) \rightarrow L^{p}(a M \mathrm{~d} v) \\
\text { is Fredholm for every } p \in(1, \infty)
\end{array}\right.
$$


This assertion follows from the decomposition $\frac{1}{a} \mathcal{L}=\mathcal{I}+\mathcal{K}^{-}-2 \mathcal{K}^{+}$given by (3.11) because the operators $\mathcal{K}^{-}$and $\mathcal{K}^{+}$are compact by (3.23) and (3.27).

3.5. Fifth assumption. Our fifth assumption regarding $b$ will only be used in section 9 to yield some compactness. It is stated in (3.29) :

$$
\bar{b}_{H}\left(v_{1}-v\right) \leq C\left(1+a_{H}\left(v_{1}\right)\right)\left(1+a_{H}(v)\right) \text { for every } v_{1}, v \in \mathbb{R}^{D},
$$

where $a_{H}(v)$ and $\bar{b}_{H}(v)$ are defined by

$$
a_{H}(v)=\int_{\mathbb{R}^{D}} \bar{b}_{H}\left(v_{1}-v\right) M_{1} \mathrm{~d} v_{1}=\iint_{\mathbb{S}^{D-1} \times \mathbb{R}^{D}} b_{H}\left(\omega, v_{1}-v\right) \mathrm{d} \omega M_{1} \mathrm{~d} v_{1}
$$

and

$$
\begin{array}{ll}
b_{H}(\omega, v)=b(\omega, v), & \text { for }|v|>V_{0} \\
b_{H}(\omega, v)=\min \{b(\omega, v), C\}, & \text { for }|v| \leq V_{0},
\end{array}
$$

for some constants $C$ and $V_{0}$. We point out that for soft potential $\bar{b}_{H}$ is bounded and hence (3.29) holds. Also, (3.29) is a simple consequence of (3.15) if $s=\infty$. This assumption is hence satisfied by all classical collision kernel. It is only needed in section 9 (see also the remark in subsection 3.7).

3.6. Null Spaces. Here we characterize the null space of the Fredholm operator $\frac{1}{a} \mathcal{L}$ considered over $L^{p}(a M \mathrm{~d} v)$ for every $p \in(1, \infty)$. One can use the collisional symmetries (2.14) to show that $\frac{1}{a} \mathcal{L}$ is formally symmetric and nonnegative definite with respect to the $L^{2}(a M \mathrm{~d} v)$ inner product. In particular, for every $\tilde{g} \in L^{2}(a M \mathrm{~d} v)$ one shows that

$$
\langle\tilde{g} \mathcal{L} \tilde{g}\rangle=\frac{1}{4}\left\langle\left(\tilde{g}+\tilde{g}_{1}-\tilde{g}^{\prime}-\tilde{g}_{1}^{\prime}\right)^{2}\right\rangle \geq 0 .
$$

It can be shown from this (see for example [11], Chapter IV.1) that the null space of $\frac{1}{a} \mathcal{L}: L^{2}(a M \mathrm{~d} v) \rightarrow L^{2}(a M \mathrm{~d} v)$ is $\operatorname{span}\left\{1, v_{1}, \cdots, v_{D},|v|^{2}\right\}$. Our first, third, and fourth assumptions combine to show moreover that for every $p \in(1, \infty)$

$$
\left\{\begin{array}{l}
\text { the null space of } \frac{1}{a} \mathcal{L}: L^{p}(a M \mathrm{~d} v) \rightarrow L^{p}(a M \mathrm{~d} v) \\
\text { is given by } \operatorname{Null}(\mathcal{L}) \equiv \operatorname{span}\left\{1, v_{1}, \cdots, v_{D},|v|^{2}\right\} .
\end{array}\right.
$$

Indeed, because our first assumption (3.4) implies that $\operatorname{Null}(\mathcal{L}) \subset$ $L^{p}(a M \mathrm{~d} v)$ for every $p \in[1, \infty)$, it is clear that $\operatorname{Null}(\mathcal{L})$ is contained in the null space of $\frac{1}{a} \mathcal{L}: L^{p}(a M \mathrm{~d} v) \rightarrow L^{p}(a M \mathrm{~d} v)$ for every $p \in[1, \infty)$. Because $L^{p}(a M \mathrm{~d} v) \subset L^{2}(a M \mathrm{~d} v)$ for every $p \in[2, \infty)$, and because the null space of $\frac{1}{a} \mathcal{L}: L^{2}(a M \mathrm{~d} v) \rightarrow L^{2}(a M \mathrm{~d} v)$ is given by $\operatorname{Null}(\mathcal{L})$, it therefore follows that the null space of $\frac{1}{a} \mathcal{L}: L^{p}(a M \mathrm{~d} v) \rightarrow L^{p}(a M \mathrm{~d} v)$ 
is also given by $\operatorname{Null}(\mathcal{L})$ for every $p \in[2, \infty)$. Now observe that the adjoint of $\frac{1}{a} \mathcal{L}$ over $L^{p}(a M \mathrm{~d} v)$ is $\frac{1}{a} \mathcal{L}$ over $L^{p^{*}}(a M \mathrm{~d} v)$. Because by (3.28) these operators are Fredholm, their null spaces must have the same dimension. In particular, when $p \in(1,2]$ the dimension of the null space must be equal to the dimension of $\operatorname{Null}(\mathcal{L})$. It therefore follows that the null space of $\frac{1}{a} \mathcal{L}: L^{p}(a M \mathrm{~d} v) \rightarrow L^{p}(a M \mathrm{~d} v)$ is also given by $\operatorname{Null}(\mathcal{L})$ for every $p \in(1,2]$.

3.7. Coercivity. We will make use of some coercivity estimates the operator $\mathcal{L}$ satisfies. If we let $\lambda>0$ be the smallest nonzero eigenvalue of $\frac{1}{a} \mathcal{L}$ considered over $L^{2}(a M \mathrm{~d} v)$ then one has the coercivity estimate

$$
\lambda\left\langle a\left(\mathcal{P}_{a}^{\perp} \tilde{g}\right)^{2}\right\rangle \leq\langle\tilde{g} \mathcal{L} \tilde{g}\rangle \quad \text { for every } \tilde{g} \in L^{2}(a M \mathrm{~d} v) .
$$

Here $\mathcal{P}_{a}^{\perp}=\mathcal{I}-\mathcal{P}_{a}$ and $\mathcal{P}_{a}$ is the orthogonal projection from $L^{2}(a M \mathrm{~d} v)$ onto $\operatorname{Null}(\mathcal{L})$, which is given by

$$
\begin{aligned}
\mathcal{P}_{a} \tilde{g}=\langle a \tilde{g}\rangle & +\frac{1}{\frac{1}{D}\left\langle a|v|^{2}\right\rangle} v \cdot\langle a v \tilde{g}\rangle \\
& +\frac{|v|^{2}-\left\langle a|v|^{2}\right\rangle}{\left\langle a|v|^{4}\right\rangle-\left\langle a|v|^{2}\right\rangle^{2}}\left\langle\left(|v|^{2}-\left\langle a|v|^{2}\right\rangle\right) \tilde{g}\right\rangle .
\end{aligned}
$$

This follows from the Fredholm property (3.28), the fact that $\frac{1}{a} \mathcal{L}$ is symmetric, and the characterization of $\operatorname{Null}(\mathcal{L})$ given by $(3.33)$.

One can show that for some $\ell>0$ the operator $\mathcal{L}$ satisfies the coercivity estimate

$$
\ell\left\langle a\left(\mathcal{P}^{\perp} \tilde{g}\right)^{2}\right\rangle \leq\langle\tilde{g} \mathcal{L} \tilde{g}\rangle \quad \text { for every } \tilde{g} \in L^{2}(a M \mathrm{~d} v) .
$$

Here $\mathcal{P}^{\perp}=\mathcal{I}-\mathcal{P}$ and $\mathcal{P}$ is the orthogonal projection from $L^{2}(M \mathrm{~d} v)$ onto $\operatorname{Null}(\mathcal{L})$, which is given by

$$
\mathcal{P} \tilde{g}=\langle\tilde{g}\rangle+v \cdot\langle v \tilde{g}\rangle+\left(\frac{1}{2}|v|^{2}-\frac{D}{2}\right)\left\langle\left(\frac{1}{D}|v|^{2}-1\right) \tilde{g}\right\rangle .
$$

Indeed, assumption (3.9) ensures that $\mathcal{P}$ and $\mathcal{P}^{\perp}$ are bounded as linear operators from $L^{2}(a M \mathrm{~d} v)$ into itself. Because $\mathcal{P}^{\perp}=\mathcal{P}^{\perp} \mathcal{P}_{a}^{\perp}$, we then have that every $\tilde{g} \in L^{2}(a M \mathrm{~d} v)$ satisfies

$$
\left\|\mathcal{P}^{\perp} \tilde{g}\right\|_{L^{2}(a M \mathrm{~d} v)}=\left\|\mathcal{P}^{\perp} \mathcal{P}_{a}^{\perp} \tilde{g}\right\|_{L^{2}(a M \mathrm{~d} v)} \leq\left\|\mathcal{P}^{\perp}\right\|_{L^{2}(a M \mathrm{~d} v)}\left\|\mathcal{P}_{a}^{\perp} \tilde{g}\right\|_{L^{2}(a M \mathrm{~d} v)},
$$

where $\left\|\mathcal{P}^{\perp}\right\|_{L^{2}(a M \mathrm{~d} v)}$ denotes the operator norm of $\mathcal{P}^{\perp}$. It therefore follows from (3.34) that we may take $\ell=\lambda /\left\|\mathcal{P}^{\perp}\right\|_{L^{2}(a M \mathrm{~d} v)}^{2}$ in (3.36).

Remark We will also use the operator

$$
\frac{1}{a_{H}} \mathcal{L}_{H}: L^{2}\left(a_{H} M \mathrm{~d} v\right) \rightarrow L^{2}\left(a_{H} M \mathrm{~d} v\right)
$$


where we denote

(3.39) $\mathcal{L}_{H} \tilde{g} \equiv-2 \mathcal{Q}_{H}(1, \tilde{g})=\iint_{\mathbb{S}^{D-1} \times \mathbb{R} D}\left(\tilde{g}+\tilde{g}_{1}-\tilde{g}^{\prime}-\tilde{g}_{1}^{\prime}\right) b_{H} \mathrm{~d} \omega M_{1} \mathrm{~d} v_{1}$.

The null space and coercivity properties also hold for the operator $\mathcal{L}_{H}$ if we take $C$ big enough in (3.31). Indeed, since $L^{2}\left(a_{H} M \mathrm{~d} v\right)=$ $L^{2}(a M \mathrm{~d} v)$, we just have to observe that $\frac{1}{a_{H}} \mathcal{L}_{H}$ goes to $\frac{1}{a} \mathcal{L}$ in the operator norm when $C$ goes to infinity.

3.8. Pseudo-Inverse. We use a particular pseudo inverse of $\mathcal{L}$ defined as follows. The Fredholm property (3.28) implies that for every $p \in$ $(1, \infty)$

$$
\mathcal{L}: L^{p}(a M \mathrm{~d} v) \rightarrow L^{p}\left(a^{1-p} M \mathrm{~d} v\right) \quad \text { is bounded },
$$

and that for every $\xi \in L^{p}\left(a^{1-p} M \mathrm{~d} v\right)$ there exists a unique $\hat{\xi} \in L^{p}(a M \mathrm{~d} v)$ such that

$$
\mathcal{L} \hat{\xi}=\mathcal{P}^{\perp} \xi, \quad \mathcal{P} \hat{\xi}=0 .
$$

For every $\xi \in L^{p}\left(a^{1-p} M \mathrm{~d} v\right)$ we define $\mathcal{L}^{-1} \xi=\hat{\xi}$ where $\hat{\xi}$ is determined above. This defines an operator $\mathcal{L}^{-1}$ such that

$$
\begin{aligned}
\mathcal{L}^{-1}: & L^{p}\left(a^{1-p} M \mathrm{~d} v\right) \rightarrow L^{p}(a M \mathrm{~d} v) \quad \text { is bounded }, \\
& \mathcal{L}^{-1} \mathcal{L}=\mathcal{P}^{\perp} \quad \text { over } L^{p}(a M \mathrm{~d} v), \\
& \mathcal{L} \mathcal{L}^{-1}=\mathcal{P}^{\perp} \quad \text { over } L^{p}\left(a^{1-p} M \mathrm{~d} v\right),
\end{aligned}
$$

and $\operatorname{Null}\left(\mathcal{L}^{-1}\right)=\operatorname{Null}(\mathcal{L})$. The operator $\mathcal{L}^{-1}$ is the unique pseudo inverse of $\mathcal{L}$ with these properties. 


\section{Formal Derivation of the Navier-Stokes-Fourier SYSTEM}

The Navier-Stokes-Fourier system (1.1-1.2) can be formally derived from the Boltzmann equation through a scaling in which the fluctuations of the kinetic density $F$ about the absolute Maxwellian $M$ are scaled to be on the order of $\epsilon$. More precisely, we consider families of solutions $G_{\epsilon}$ to the scaled Boltzmann initial-value problem (2.7) that are parametrized by the Knudsen number $\epsilon$ and that have the form

$$
G_{\epsilon}^{i n}=1+\epsilon g_{\epsilon}^{i n}, \quad G_{\epsilon}=1+\epsilon g_{\epsilon},
$$

One sees directly from the Boltzmann equation (2.7) satisfied by $G_{\epsilon}$ that the fluctuations $g_{\epsilon}$ satisfy

$$
\epsilon \partial_{t} g_{\epsilon}+v \cdot \nabla_{x} g_{\epsilon}+\frac{1}{\epsilon} \mathcal{L} g_{\epsilon}=\mathcal{Q}\left(g_{\epsilon}, g_{\epsilon}\right) .
$$

We assume that $g_{\epsilon} \rightarrow g$ formally, where $g \in L^{\infty}\left(\mathrm{d} t ; L^{2}(M \mathrm{~d} v \mathrm{~d} x)\right)$, and that all formally small terms vanish.

4.1. Step One. The first step shows that the limit $g$ is an infinitesimal Maxwellian. Upon multiplying (4.2) by $\epsilon$ and letting $\epsilon \rightarrow 0$, one finds that $\mathcal{L} g=0$. Because $\operatorname{Null}(\mathcal{L})=\operatorname{span}\left\{1, v_{1}, \cdots, v_{D},|v|^{2}\right\}$ and because the limit $g$ is assumed to belong to $L^{\infty}\left(\mathrm{d} t ; L^{2}(M \mathrm{~d} v \mathrm{~d} x)\right)$, we conclude that $g$ has the form of a so-called infinitesimal Maxwellian - namely, that

$$
g=\rho+v \cdot u+\left(\frac{1}{2}|v|^{2}-\frac{D}{2}\right) \theta,
$$

for some $(\rho, u, \theta)$ in $L^{\infty}\left(\mathrm{d} t ; L^{2}\left(\mathrm{~d} x ; \mathbb{R} \times \mathbb{R}^{D} \times \mathbb{R}\right)\right)$.

4.2. Step Two. The second step shows that $(\rho, u, \theta)$ satisfies the incompressibility and Boussinesq relations. Observe from (2.15) that the fluctuations $g_{\epsilon}$ formally satisfy the local conservation laws

$$
\begin{aligned}
\epsilon \partial_{t}\left\langle g_{\epsilon}\right\rangle+\nabla_{x} \cdot\left\langle v g_{\epsilon}\right\rangle & =0, \\
\epsilon \partial_{t}\left\langle v g_{\epsilon}\right\rangle+\nabla_{x} \cdot\left\langle v \otimes v g_{\epsilon}\right\rangle & =0, \\
\epsilon \partial_{t}\left\langle\frac{1}{2}|v|^{2} g_{\epsilon}\right\rangle+\nabla_{x} \cdot\left\langle v \frac{1}{2}|v|^{2} g_{\epsilon}\right\rangle & =0 .
\end{aligned}
$$

By letting $\epsilon \rightarrow 0$ in these equations and using the infinitesimal Maxwellian form of $g$ given by (4.3), one finds that

$$
\nabla_{x} \cdot u=0, \quad \nabla_{x}(\rho+\theta)=0 .
$$

The first equation is the incompressibility relation, while the second says $\rho+\theta$ is a function of time only. Upon then letting $\epsilon \rightarrow 0$ in the 
global energy conservation law of (2.16), one thereby concludes that

$$
\rho+\theta=\int_{\mathbb{T}^{D}}(\rho+\theta) \mathrm{d} x=\frac{2}{D} \int_{\mathbb{T}^{D}}\left\langle\frac{1}{2}|v|^{2} g\right\rangle \mathrm{d} x=0 .
$$

Hence, $(\rho, u, \theta)$ satisfy the incompressibility and Boussinesq relations (1.1). The Boussinesq relation implies that the infinitesimal Maxwellian form (4.3) of $g$ reduces to

$$
g=v \cdot u+\left(\frac{1}{2}|v|^{2}-\frac{D+2}{2}\right) \theta,
$$

for some $(u, \theta)$ in $L^{\infty}\left(\mathrm{d} t ; L^{2}\left(\mathrm{~d} x ; \mathbb{R}^{D} \times \mathbb{R}\right)\right)$.

4.3. The Key Idea. The next three steps show that the evolution of $(u, \theta)$ is governed by the motion and heat equations. The difficulty here is that when the local conservation laws (4.4) are written so that the time derivatives are order 1 , the fluxes become order $1 / \epsilon$. This difficulty is overcome by the following strategy [2]. Observe that the momentum and a linear combination of the mass and energy local conservation laws from (4.4) can be expressed as

$$
\begin{aligned}
\partial_{t}\left\langle v g_{\epsilon}\right\rangle+\frac{1}{\epsilon} \nabla_{x} \cdot\left\langle A g_{\epsilon}\right\rangle+\frac{1}{\epsilon} \nabla_{x}\left\langle\frac{1}{D}|v|^{2} g_{\epsilon}\right\rangle & =0, \\
\partial_{t}\left\langle\left(\frac{1}{2}|v|^{2}-\frac{D+2}{2}\right) g_{\epsilon}\right\rangle+\frac{1}{\epsilon} \nabla_{x} \cdot\left\langle B g_{\epsilon}\right\rangle & =0,
\end{aligned}
$$

where the matrix-valued function $A$ and the vector-valued function $B$ are defined by

$$
A(v)=v \otimes v-\frac{1}{D}|v|^{2} I, \quad B(v)=\frac{1}{2}|v|^{2} v-\frac{D+2}{2} v .
$$

As is common when studying incompressible fluid dynamical limits, the momentum equation will be integrated against divergence-free test functions. The last term in its flux will thereby be eliminated, and one only has to pass to the limit in the flux terms of (4.6) that involve $A$ and $B$ - namely, in the terms

$$
\frac{1}{\epsilon}\left\langle A g_{\epsilon}\right\rangle, \quad \frac{1}{\epsilon}\left\langle B g_{\epsilon}\right\rangle .
$$

There is a chance that these terms have a limit because each entry of $A$ and $B$ is in $\operatorname{Null}(\mathcal{L})^{\perp}$ while $g_{\epsilon}$ converges to $g$, which is in $\operatorname{Null}(\mathcal{L})$. The next two steps show that these terms indeed have a formal limit.

4.4. Step Three. The third step evaluates the limit for moments of the form $\left\langle\mathcal{L} \hat{\xi} g_{\epsilon}\right\rangle / \epsilon$ for every $\hat{\xi} \in L^{2}(a M \mathrm{~d} v)$. Because $\mathcal{L}$ is formally symmetric, one has

$$
\left\langle\mathcal{L} \hat{\xi} g_{\epsilon}\right\rangle=\left\langle\hat{\xi} \mathcal{L} g_{\epsilon}\right\rangle
$$


Upon multiplying (4.2) by $\hat{\xi}$ and integrating, one sees that the fluctuations $g_{\epsilon}$ formally satisfy the general moment equation

$$
\epsilon \partial_{t}\left\langle\hat{\xi} g_{\epsilon}\right\rangle+\nabla_{x} \cdot\left\langle v \hat{\xi} g_{\epsilon}\right\rangle+\frac{1}{\epsilon}\left\langle\hat{\xi} \mathcal{L} g_{\epsilon}\right\rangle=\left\langle\hat{\xi} \mathcal{Q}\left(g_{\epsilon}, g_{\epsilon}\right)\right\rangle
$$

Upon formally letting $\epsilon \rightarrow 0$ in this equation, one finds that, in the sense of distributions,

$$
\frac{1}{\epsilon}\left\langle\mathcal{L} \hat{\xi} g_{\epsilon}\right\rangle \rightarrow\langle\hat{\xi} \mathcal{Q}(g, g)\rangle-\left\langle\hat{\xi} v \cdot \nabla_{x} g\right\rangle
$$

Because $g$ has the infinitesimal Maxwellian form given by (4.5), one can show [2] that

$$
\langle\hat{\xi} \mathcal{Q}(g, g)\rangle=\frac{1}{2}\left\langle\hat{\xi} \mathcal{L}\left(g^{2}\right)\right\rangle=\frac{1}{2}\left\langle\mathcal{L} \hat{\xi} \mathcal{P}^{\perp}\left(g^{2}\right)\right\rangle,
$$

where $\mathcal{P}^{\perp}=\mathcal{I}-\mathcal{P}$ and $\mathcal{P}$ is the orthogonal projection from $L^{2}(M \mathrm{~d} v)$ onto $\operatorname{Null}(\mathcal{L})$, which is given by $(3.37)$.

Given (4.12), one can again use the infinitesimal Maxwellian form given by (4.5) to show moreover that (4.11) becomes

$$
\begin{aligned}
\frac{1}{\epsilon}\left\langle\mathcal{L} \hat{\xi} g_{\epsilon}\right\rangle \rightarrow & \frac{1}{2}\langle\mathcal{L} \hat{\xi} A\rangle: u \otimes u+\langle\mathcal{L} \hat{\xi} B\rangle \cdot u \theta+\frac{1}{2}\langle\mathcal{L} \hat{\xi} C\rangle \theta^{2} \\
& -\langle\hat{\xi} A\rangle: \nabla_{x} u-\langle\hat{\xi} B\rangle \cdot \nabla_{x} \theta .
\end{aligned}
$$

where the matrix-valued function $A$ and the vector-valued function $B$ are defined by (4.7), while the scalar-valued function $C$ is defined by

$$
C(v)=\frac{1}{4}|v|^{4}-\frac{D+2}{2}|v|^{2}+\frac{D(D+2)}{4} .
$$

One has that $C$ is in $\operatorname{Null}(\mathcal{L})^{\perp}$.

4.5. Step Four. The fourth step determines the limit of the flux terms (4.8). Because each entry of $A$ and $B$ is in $L^{2}\left(a^{-1} M \mathrm{~d} v\right)$, we let $\widehat{A} \in$ $L^{2}\left(a M \mathrm{~d} v ; \mathbb{R}^{D \times D}\right)$ and $\widehat{B} \in L^{2}\left(a M \mathrm{~d} v ; \mathbb{R}^{D}\right)$ be given by

$$
\widehat{A}=\mathcal{L}^{-1} A, \quad \text { and } \quad \widehat{B}=\mathcal{L}^{-1} B .
$$

Because $\mathcal{P} A=0$ and $\mathcal{P} B=0$, these are the unique solutions of

$$
\begin{array}{ll}
\mathcal{L} \widehat{A}=A, & \mathcal{P} \widehat{A}=0, \\
\mathcal{L} \widehat{B}=B, & \mathcal{P} \widehat{B}=0 .
\end{array}
$$

Because each entry of $A$ and $B$ is in $L^{p}\left(a^{1-p} M \mathrm{~d} v\right)$ for every $p \in(1, \infty)$, each entry of $\widehat{A}$ and $\widehat{B}$ is therefore in $L^{p}(a M \mathrm{~d} v)$ for every $p \in(1, \infty)$. 
By letting $\hat{\xi}$ in (4.13) be the entries of $\widehat{A}$ and $\widehat{B}$ and using the facts $A$ and $\widehat{A}$ are even, $B$ and $\widehat{B}$ are odd, and $\langle A C\rangle=0$, one finds that

$$
\begin{aligned}
\frac{1}{\epsilon}\left\langle A g_{\epsilon}\right\rangle & \rightarrow \frac{1}{2}\langle A \otimes A\rangle:(u \otimes u)-\langle\widehat{A} \otimes A\rangle: \nabla_{x} u \\
& =u \otimes u-\frac{1}{D}|u|^{2} I-\nu\left(\nabla_{x} u+\left(\nabla_{x} u\right)^{T}\right), \\
\frac{1}{\epsilon}\left\langle B g_{\epsilon}\right\rangle & \rightarrow\langle B \otimes B\rangle \cdot u \theta-\langle\widehat{B} \otimes B\rangle \cdot \nabla_{x} \theta \\
& =\frac{D+2}{2} u \theta-\kappa \nabla_{x} \theta,
\end{aligned}
$$

where kinematic viscosity $\nu$ and thermal conductivity $\kappa$ are given by

$$
\nu=\frac{1}{(D-1)(D+2)}\langle\widehat{A}: \mathcal{L} \widehat{A}\rangle, \quad \kappa=\frac{1}{D}\langle\widehat{B} \cdot \mathcal{L} \widehat{B}\rangle .
$$

4.6. Step Five. The fifth step shows that the evolution of $(u, \theta)$ is governed by the motion and heat equations (1.2). The fluctuations $g_{\epsilon}$ formally satisfy the local conservation laws (4.6). Hence, when letting $\epsilon \rightarrow 0$ in these equations, we use the infinitesimal Maxwellian form of $g$ given by (4.5) to evaluate the limiting densities while we use (4.17) to evaluate the limiting fluxes. We find that $(u, \theta)$ satisfies the weak form of the incompressible Navier-Stokes-Fourier dynamics (1.2). If we let $\Pi$ denote the orthogonal projection from $L^{2}\left(\mathrm{~d} x ; \mathbb{R}^{D}\right)$ onto divergence-free vector fields, then by the formal continuity in time of the densities in (4.6), one sees that

$$
\left(u^{i n}, \theta^{i n}\right)=\lim _{\epsilon \rightarrow 0}\left(\Pi\left\langle v g_{\epsilon}^{i n}\right\rangle,\left\langle\left(\frac{1}{D+2}|v|^{2}-1\right) g_{\epsilon}^{i n}\right\rangle\right),
$$

provided we assume that the limit on the right-hand side exists in the sense of distributions for some $\left(u^{i n}, \theta^{i n}\right) \in L^{2}\left(\mathrm{~d} x ; \mathbb{R}^{D} \times \mathbb{R}\right)$.

4.7. Step Six. The sixth step determines the limit of the difference of $g_{\epsilon}$ from its infinitesimal Maxwellian, $\mathcal{P} g_{\epsilon}$. The Fredholm alternative implies that for every $\xi \in L^{2}\left(a^{-1} M \mathrm{~d} v\right)$ there is a unique $\hat{\xi} \in L^{2}(a M \mathrm{~d} v)$ that solves $\mathcal{L} \hat{\xi}=\mathcal{P}^{\perp} \xi$ with $\mathcal{P} \hat{\xi}=0$. Hence, for every $\xi \in L^{2}(M \mathrm{~d} v)$ one has

$$
\left\langle\xi \mathcal{P}^{\perp} g_{\epsilon}\right\rangle=\left\langle g_{\epsilon} \mathcal{P}^{\perp} \xi\right\rangle=\left\langle g_{\epsilon} \mathcal{L} \mathcal{L}^{-1} \xi\right\rangle=\left\langle g_{\epsilon} \mathcal{L} \hat{\xi}\right\rangle,
$$

One thereby sees from (4.13) that as $\epsilon \rightarrow 0$ one has

$$
\begin{aligned}
\frac{1}{\epsilon}\left\langle\xi \mathcal{P}^{\perp} g_{\epsilon}\right\rangle \rightarrow & \frac{1}{2}\langle\mathcal{L} \hat{\xi} A\rangle: u \otimes u+\langle\mathcal{L} \hat{\xi} B\rangle \cdot u \theta+\frac{1}{2}\langle\mathcal{L} \hat{\xi} C\rangle \theta^{2} \\
& -\langle\hat{\xi} A\rangle: \nabla_{x} u-\langle\hat{\xi} B\rangle \cdot \nabla_{x} \theta \\
= & \frac{1}{2}\langle\xi A\rangle: u \otimes u+\langle\xi B\rangle \cdot u \theta+\frac{1}{2}\langle\xi C\rangle \theta^{2} \\
& -\langle\xi \widehat{A}\rangle: \nabla_{x} u-\langle\xi \widehat{B}\rangle \cdot \nabla_{x} \theta .
\end{aligned}
$$


Hence, as $\epsilon \rightarrow 0$ one has the distribution limit

$$
\begin{gathered}
\frac{1}{\epsilon} \mathcal{P}^{\perp} g_{\epsilon} \rightarrow \frac{1}{2} A: u \otimes u+B \cdot u \theta+\frac{1}{2} C \theta^{2} \\
-\widehat{A}: \nabla_{x} u-\widehat{B} \cdot \nabla_{x} \theta .
\end{gathered}
$$

The right-hand side is exactly the first correction to the infinitesimal Maxwellian that one obtains from the Chapman-Enskog expansion with the incompressible Navier-Stokes-Fourier scaling.

4.8. Formal Navier-Stokes-Fourier Limit Theorem. The above formal derivation can be stated more precisely as follows.

Theorem 4.1. (Formal Theorem) Let $b$ be a collision kernel that satisfies the assumptions of Section 3.

Let $G_{\epsilon}$ be a family of distribution solutions of the scaled Boltzmann initial-value problem (2.7) with initial data $G_{\epsilon}^{\text {in }}$ that satisfy the normalizations (2.11). Let $G_{\epsilon}^{\text {in }}$ and $G_{\epsilon}$ have fluctuations $g_{\epsilon}^{\text {in }}$ and $g_{\epsilon}$ given by (4.1). Also:

(1) Assume that in the sense of distributions the family $g_{\epsilon}^{i n}$ satisfies

$$
\lim _{\epsilon \rightarrow 0}\left(\Pi\left\langle v g_{\epsilon}^{i n}\right\rangle,\left\langle\left(\frac{1}{D+2}|v|^{2}-1\right) g_{\epsilon}^{i n}\right\rangle\right)=\left(u^{i n}, \theta^{i n}\right),
$$

for some $\left(u^{i n}, \theta^{i n}\right) \in L^{2}\left(\mathrm{~d} x ; \mathbb{R}^{D} \times \mathbb{R}\right)$.

(2) Assume that for every $g_{\epsilon}$ and every $\hat{\xi} \in L^{2}(a M \mathrm{~d} v)$ the moment equation (4.10) is also satisfied in the sense of distributions.

(3) Assume that $g_{\epsilon}$ converges in the sense of distributions as $\epsilon \rightarrow 0$ to $g \in L^{\infty}\left(\mathrm{d} t ; L^{2}(M \mathrm{~d} v \mathrm{~d} x)\right)$. Assume moreover that $\mathcal{L} g_{\epsilon} \rightarrow \mathcal{L} g$, that for every $\hat{\xi} \in L^{2}(a M \mathrm{~d} v)$ the moments

$$
\left\langle\hat{\xi} g_{\epsilon}\right\rangle, \quad\left\langle v \hat{\xi} g_{\epsilon}\right\rangle, \quad\left\langle\hat{\xi} \mathcal{Q}\left(g_{\epsilon}, g_{\epsilon}\right)\right\rangle
$$

converge respectively to

$$
\langle\hat{\xi} g\rangle, \quad\langle v \hat{\xi} g\rangle, \quad\langle\hat{\xi} \mathcal{Q}(g, g)\rangle,
$$

and that every formally small term vanishes, all in the sense of distributions as $\epsilon \rightarrow 0$.

Then $g$ is a local infinitesimal Maxwellian (4.5) where $(u, \theta)$ is a weak solution of the Navier-Stokes-Fourier system (1.1-1.2) with $\nu$ and $\kappa$ given by (4.18) and with initial data $\left(u^{\text {in }}, \theta^{\text {in }}\right)$ given by (4.22). Moreover, the family $\mathcal{P}^{\perp} g_{\epsilon}$ of the deviations of $g_{\epsilon}$ from the infinitesimal Maxwellians satisfies the limit (4.21) in the sense of distributions. 


\section{Global Solutions}

In order to mathematically justify the formal fluid dynamical limit described in the last section, we must make precise: (1) the notion of solution for the Boltzmann equation, and (2) the notion of solution for the Navier-Stokes-Fourier system. Ideally, these solutions should be global while the bounds should be physically natural. We therefore work in the setting of DiPerna-Lions renormalized solutions for the Boltzmann equation, and in the setting of Leray solutions for the Navier-Stokes-Fourier system. These theories have the virtues of considering physically natural classes of initial data, and consequently, of yielding global solutions.

5.1. DiPerna-Lions Solutions. DiPerna and Lions [15] proved the global existence of a type of weak solution to the Boltzmann equation over the whole space $\mathbb{R}^{D}$ for any initial data satisfying natural physical bounds. As they pointed out, with only slight modifications their theory can be extended to the periodic box $\mathbb{T}^{D}$. Their original theory has been strengthened, most notably in [31] and [37]. Here we give a version of their theory relevant to this paper.

The DiPerna-Lions theory does not yield solutions that are known to solve the Boltzmann equation in the usual weak sense. Rather, it gives the existence of a global weak solution to a class of formally equivalent initial-value problems that are obtained by multiplying the Boltzmann equation in $(2.7)$ by $\Gamma^{\prime}(G)$, where $\Gamma^{\prime}$ is the derivative of an admissible function $\Gamma$ :

$$
\begin{gathered}
\left(\epsilon \partial_{t}+v \cdot \nabla_{x}\right) \Gamma(G)=\frac{1}{\epsilon} \Gamma^{\prime}(G) \mathcal{Q}(G, G), \\
G(v, x, 0)=G^{i n}(v, x) \geq 0 .
\end{gathered}
$$

A function $\Gamma:[0, \infty) \rightarrow \mathbb{R}$ is called admissible if it is continuously differentiable and for some constant $C_{\Gamma}<\infty$ its derivative satisfies

$$
\left|\Gamma^{\prime}(Z)\right| \leq \frac{C_{\Gamma}}{\sqrt{1+Z}} \quad \text { for every } Z \geq 0
$$

The solutions lie in $C\left([0, \infty) ; w-L^{1}(M \mathrm{~d} v \mathrm{~d} x)\right)$, where the prefix " $w$-" on a space indicates that the space is endowed with its weak topology. We say that $G \geq 0$ is a weak solution of (5.1) provided that it is initially equal to $G^{i n}$, and that it satisfies (5.1) in the sense that for 
every $Y \in L^{\infty}\left(\mathrm{d} v ; C^{1}\left(\mathbb{T}^{D}\right)\right)$ and every $\left[t_{1}, t_{2}\right] \subset[0, \infty)$ it satisfies

$$
\begin{aligned}
\epsilon \int_{\mathbb{T}^{D}}\left\langle\Gamma\left(G\left(t_{2}\right)\right) Y\right\rangle \mathrm{d} x & -\epsilon \int_{\mathbb{T}^{D}}\left\langle\Gamma\left(G\left(t_{1}\right)\right) Y\right\rangle \mathrm{d} x \\
& -\int_{t_{1}}^{t_{2}} \int_{\mathbb{T}^{D}}\left\langle\Gamma(G) v \cdot \nabla_{x} Y\right\rangle \mathrm{d} x \mathrm{~d} t \\
& =\frac{1}{\epsilon} \int_{t_{1}}^{t_{2}} \int_{\mathbb{T}^{D}}\left\langle\Gamma^{\prime}(G) \mathcal{Q}(G, G) Y\right\rangle \mathrm{d} x \mathrm{~d} t .
\end{aligned}
$$

If $G$ is a weak solution of (5.1) for one such $\Gamma$ with $\Gamma^{\prime}>0$, and if $G$ satisfies certain bounds, then it is a weak solution of (5.1) for every admissible $\Gamma$. Such solutions are called renormalized solutions of the Boltzmann equation (2.7).

Specifically, cast in our setting, the theory of renormalized solutions yields the following.

Theorem 5.1. (DiPerna-Lions Renormalized Solutions) Let b satisfy

$$
\begin{gathered}
\lim _{|v| \rightarrow \infty} \frac{1}{1+|v|^{2}} \int_{\mathbb{S}^{D-1} \times K} b\left(\omega, v_{1}-v\right) \mathrm{d} \omega \mathrm{d} v_{1}=0, \\
\text { for every compact } K \subset \mathbb{R}^{D} .
\end{gathered}
$$

Given any initial data $G^{\text {in }}$ in the entropy class

$$
E(M \mathrm{~d} v \mathrm{~d} x)=\left\{G^{i n} \geq 0: H\left(G^{i n}\right)<\infty\right\},
$$

there exists at least one $G \geq 0$ in $C\left([0, \infty) ; w-L^{1}(M \mathrm{~d} v \mathrm{~d} x)\right)$ that for every admissible function $\Gamma$ is a weak solution of (5.1). This solution satisfies a weak form of the local conservation law of mass

$$
\epsilon \partial_{t}\langle G\rangle+\nabla_{x} \cdot\langle v G\rangle=0 .
$$

Moreover, there exists a matrix-valued distribution $W$ such that $W \mathrm{~d} x$ is nonnegative definite measure and $G$ and $W$ satisfy a weak form of the local conservation law of momentum

$$
\epsilon \partial_{t}\langle v G\rangle+\nabla_{x} \cdot\langle v \otimes v G\rangle+\nabla_{x} \cdot W=0,
$$

and for every $t>0$, the global energy equality

$$
\int_{\mathbb{T}^{D}}\left\langle\frac{1}{2}|v|^{2} G(t)\right\rangle \mathrm{d} x+\int_{\mathbb{T}^{D}} \frac{1}{2} \operatorname{tr}(W(t)) \mathrm{d} x=\int_{\mathbb{T}^{D}}\left\langle\frac{1}{2}|v|^{2} G^{i n}\right\rangle \mathrm{d} x,
$$

and the global entropy inequality

$$
H(G(t))+\int_{\mathbb{T}^{D}} \frac{1}{2} \operatorname{tr}(W(t)) \mathrm{d} x+\frac{1}{\epsilon^{2}} \int_{0}^{t} R(G(s)) \mathrm{d} s \leq H\left(G^{i n}\right) .
$$


DiPerna-Lions renormalized solutions are not known to satisfy many properties that one would formally expect to be satisfied by solutions of the Boltzmann equation. In particular, the theory does not assert either the local conservation of momentum in (2.15), the global conservation of energy in (2.16), the global entropy equality (2.18), or even a local entropy inequality; nor does it assert the uniqueness of the solution. Nevertheless, as shown in [25], it provides enough control to establish a Navier-Stokes-Fourier limit theorem for bounded collision kernels and, as shown here, to do so for a much larger class of collision kernels.

5.2. Leray Solutions. The DiPerna-Lions theory has many similarities with the Leray theory of global weak solutions of the initial-value problem for Navier-Stokes type systems [29]. For the Navier-StokesFourier system (1.1-1.2) with mean zero initial data, we set the Leray theory in the following Hilbert spaces of vector- and scalar-valued functions:

$$
\begin{aligned}
& \mathbb{H}_{v}=\left\{w \in L^{2}\left(\mathrm{~d} x ; \mathbb{R}^{D}\right): \nabla_{x} \cdot w=0, \int w \mathrm{~d} x=0\right\}, \\
& \mathbb{H}_{s}=\left\{\chi \in L^{2}(\mathrm{~d} x ; \mathbb{R}): \int \chi \mathrm{d} x=0\right\}, \\
& \mathbb{V}_{v}=\left\{w \in \mathbb{H}_{v}: \int\left|\nabla_{x} w\right|^{2} \mathrm{~d} x<\infty\right\}, \\
& \mathbb{V}_{s}=\left\{\chi \in \mathbb{H}_{s}: \int\left|\nabla_{x} \chi\right|^{2} \mathrm{~d} x<\infty\right\} .
\end{aligned}
$$

Let $\mathbb{H}=\mathbb{H}_{v} \oplus \mathbb{H}_{s}$ and $\mathbb{V}=\mathbb{V}_{v} \oplus \mathbb{V}_{s}$.

Specifically, cast in our setting, the Leray theory yields the following.

Theorem 5.2. (Leray Solutions) Given any initial data $\left(u^{i n}, \theta^{i n}\right) \in \mathbb{H}$, there exists at least one $(u, \theta) \in C([0, \infty) ; w-\mathbb{H}) \cap L^{2}(\mathrm{~d} t ; \mathbb{V})$ that is a weak solution of the Navier-Stokes-Fourier system (1.1-1.2) in the sense that for every $(w, \chi) \in \mathbb{H} \cap C^{1}\left(\mathbb{T}^{D}\right)$ and every $\left[t_{1}, t_{2}\right] \subset[0, \infty)$ it 
satisfies

$$
\begin{aligned}
\int w \cdot u\left(t_{2}\right) \mathrm{d} x & -\int w \cdot u\left(t_{1}\right) \mathrm{d} x-\int_{t_{1}}^{t_{2}} \int \nabla_{x} w:(u \otimes u) \mathrm{d} x \mathrm{~d} t \\
= & -\nu \int_{t_{1}}^{t_{2}} \int \nabla_{x} w: \nabla_{x} u \mathrm{~d} x \mathrm{~d} t \\
\int \chi \theta\left(t_{2}\right) \mathrm{d} x & -\int \chi \theta\left(t_{1}\right) \mathrm{d} x-\int_{t_{1}}^{t_{2}} \int \nabla_{x} \chi \cdot(u \theta) \mathrm{d} x \mathrm{~d} t \\
& =-\frac{2}{D+2} \kappa \int_{t_{1}}^{t_{2}} \int \nabla_{x} \chi \cdot \nabla_{x} \theta \mathrm{d} x \mathrm{~d} t .
\end{aligned}
$$

Moreover, for every $t>0,(u, \theta)$ satisfies the dissipation inequalities

$$
\begin{gathered}
\int \frac{1}{2}|u(t)|^{2} \mathrm{~d} x+\int_{0}^{t} \int \nu\left|\nabla_{x} u\right|^{2} \mathrm{~d} x \mathrm{~d} s \leq \int \frac{1}{2}\left|u^{i n}\right|^{2} \mathrm{~d} x, \\
\int \frac{D+2}{4}|\theta(t)|^{2} \mathrm{~d} x+\int_{0}^{t} \int \kappa\left|\nabla_{x} \theta\right|^{2} \mathrm{~d} x \mathrm{~d} s \leq \int \frac{D+2}{4}\left|\theta^{i n}\right|^{2} \mathrm{~d} x .
\end{gathered}
$$

By arguing formally from the Navier-Stokes-Fourier system (1.11.2), one would expect these inequalities to be equalities. However, that is not asserted by the Leray theory. Also, as was the case for the DiPerna-Lions theory, the Leray theory does not assert uniqueness of the solution.

Because the role of the dissipation inequalities (5.13) and (5.14) is to provide a-priori estimates, the existence theory also works if they are replaced by the single dissipation inequality

$$
\begin{aligned}
\int \frac{1}{2}|u(t)|^{2}+\frac{D+2}{4}|\theta(t)|^{2} \mathrm{~d} x & +\int_{0}^{t} \int \nu\left|\nabla_{x} u\right|^{2}+\kappa\left|\nabla_{x} \theta\right|^{2} \mathrm{~d} x \mathrm{~d} s \\
& \leq \int \frac{1}{2}\left|u^{i n}\right|^{2}+\frac{D+2}{4}\left|\theta^{i n}\right|^{2} \mathrm{~d} x .
\end{aligned}
$$

It is this version of the Leray theory that we will obtain in the limit. 


\section{Main Result}

In this section we state our main result, but first we recall the notion of entropic convergence, which is used in the statement of our main theorem.

6.1. Entropic Convergence. The notion of entropic convergence, introduced in [3], was used in earlier studies as a natural tool for obtaining strong convergence results for fluctuations about an absolute Maxwellian [4, 5, 18, 19, 25, 30, 34, 35]. With it, the relative entropy can be used to measure the distance of the fluctuations from their limit.

Definition 6.1. Let $G_{\epsilon}$ be a family in the entropy class $E(M \mathrm{~d} v \mathrm{~d} x)$ given by (5.5) and let $g_{\epsilon}$ be the associated family of fluctuations given by

$$
g_{\epsilon}=\frac{G_{\epsilon}-1}{\epsilon} .
$$

The family $g_{\epsilon}$ is said to converge entropically at order $\epsilon$ to some $g \in$ $L^{2}(M \mathrm{~d} v \mathrm{~d} x)$ if and only if

$$
\begin{gathered}
g_{\epsilon} \rightarrow g \text { in } w-L^{1}(M \mathrm{~d} v \mathrm{~d} x), \\
\text { and } \\
\lim _{\epsilon \rightarrow 0} \frac{1}{\epsilon^{2}} H\left(G_{\epsilon}\right)=\int_{\mathbb{T}^{D}} \frac{1}{2}\left\langle g^{2}\right\rangle \mathrm{d} x .
\end{gathered}
$$

Proposition 4.11 of [3] showed that entropic convergence is stronger than norm convergence in $\left.L^{1}(\sigma M \mathrm{~d} v \mathrm{~d} x)\right)$, where $\sigma=1+|v|^{2}$.

6.2. Statement of the Main Theorem. Our main result is the following.

Theorem 6.1. (Main Theorem.) Let the collision kernel b satisfy (3.7), (3.9), (3.15), (3.26), and (3.29).

Let $\left(u^{\text {in }}, \theta^{\text {in }}\right) \in \mathbb{H}$ and let $g^{\text {in }}$ be the local infinitesimal Maxwellian given by

$$
g^{i n}=v \cdot u^{i n}+\left(\frac{1}{2}|v|^{2}-\frac{D+2}{2}\right) \theta^{i n} .
$$

Let $G_{\epsilon}^{i n}$ be any family in the entropy class $E(M \mathrm{~d} v \mathrm{~d} x)$ given by (5.5) that satisfies the normalizations (2.11). Let $g_{\epsilon}^{\text {in }}$ be the associated family of fluctuations given by

$$
g_{\epsilon}^{i n}=\frac{G_{\epsilon}^{i n}-1}{\epsilon} .
$$

Assume that the family $g_{\epsilon}^{i n}$ satisfies

$$
g_{\epsilon}^{i n} \rightarrow g^{i n} \quad \text { entropically at order } \epsilon \text { as } \epsilon \rightarrow 0 .
$$


Let $G_{\epsilon}$ be any family of DiPerna-Lions renormalized solutions of the Boltzmann equation (2.7) that have $G_{\epsilon}^{\text {in }}$ as initial values. Let $g_{\epsilon}$ be the associated family of fluctuations given by (6.1).

Then the family $g_{\epsilon}$ is relatively compact in $w-L_{l o c}^{1}\left(\mathrm{~d} t ; w-L^{1}(\sigma M \mathrm{~d} v \mathrm{~d} x)\right)$. Every limit point $g$ of $g_{\epsilon}$ in $w-L_{l o c}^{1}\left(\mathrm{~d} t ; w-L^{1}(\sigma M \mathrm{~d} v \mathrm{~d} x)\right)$ has the infinitesimal Maxwellian form

$$
g=v \cdot u+\left(\frac{1}{2}|v|^{2}-\frac{D+2}{2}\right) \theta,
$$

where $(u, \theta) \in C([0, \infty) ; w-\mathbb{H}) \cap L^{2}(\mathrm{~d} t ; \mathbb{V})$ is a Leray solution with initial data $\left(u^{i n}, \theta^{i n}\right)$ of the Navier-Stokes-Fourier system (1.1-1.2) with $\nu$ and $\kappa$ given by (4.18). More specifically, $(u, \theta)$ satisfies the weak form of the Navier-Stokes-Fourier system given by (5.11-5.12) and the dissipation inequality (5.15).

Moreover, every subsequence $g_{\epsilon_{k}}$ of $g_{\epsilon}$ that converges to $g$ as $\epsilon_{k} \rightarrow 0$ also satisfies

$$
\begin{aligned}
& \Pi\left\langle v g_{\epsilon_{k}}\right\rangle \rightarrow u \quad \text { in } C\left([0, \infty) ; \mathcal{D}^{\prime}\left(\mathbb{T}^{D} ; \mathbb{R}^{D}\right)\right), \\
&\left\langle\left(\frac{1}{D+2}|v|^{2}-1\right) g_{\epsilon_{k}}\right\rangle \rightarrow \theta \quad \text { in } C\left([0, \infty) ; w-L^{1}(\mathrm{~d} x ; \mathbb{R})\right) .
\end{aligned}
$$

where $\Pi$ is the orthogonal projection from $L^{2}\left(\mathrm{~d} x ; \mathbb{R}^{D}\right)$ onto divergencefree vector fields.

Remark. For every $\left(u^{i n}, \theta^{i n}\right) \in \mathbb{H}$ there are families $G_{\epsilon}^{i n}$ in the entropy class $E(M \mathrm{~d} v \mathrm{~d} x)$ that satisfy the normalizations (2.11) such that the associated family of fluctuations $g_{\epsilon}^{i n}$ converges to $g^{i n}$ entropically at order $\epsilon$ as $\epsilon \rightarrow 0$. This follows from Proposition 3.4 of [5].

The above theorem still holds if we only have weak convergence initially

Corollary 6.1. Let the collision kernel b satisfy (3.7), (3.9), (3.15), (3.26), and (3.29). Let $G_{\epsilon}^{\text {in }}$ be any family in the entropy class $E(M \mathrm{~d} v \mathrm{~d} x)$ given by (5.5) that satisfies the normalizations (2.11). Let $g_{\epsilon}^{\text {in }}$ be the associated family of fluctuations. Assume that

$$
H\left(G_{\epsilon}\right) \leq C \epsilon^{2}
$$

$$
\text { and }
$$

$$
\lim _{\epsilon \rightarrow 0}\left(\Pi\left\langle v g_{\epsilon}^{i n}\right\rangle,\left\langle\left(\frac{1}{D+2}|v|^{2}-1\right) g_{\epsilon}^{i n}\right\rangle\right)=\left(u^{i n}, \theta^{i n}\right),
$$

for some $\left(u^{i n}, \theta^{i n}\right) \in L^{2}\left(\mathrm{~d} x ; \mathbb{R}^{D} \times \mathbb{R}\right)$.

Let $G_{\epsilon}$ be any family of DiPerna-Lions renormalized solutions of the Boltzmann equation (2.7) that have $G_{\epsilon}^{\text {in }}$ as initial values. Let $g_{\epsilon}$ be the associated family of fluctuations given by (6.1). 
Then, the same conclusions of the Main theorem hold a part from the convergences (6.7) and (6.8) which only hold in $w-L_{l o c}^{1}\left(\mathrm{~d} t ; w-L^{1}(\sigma M \mathrm{~d} v \mathrm{~d} x)\right)$ - Moreover, the right hand side of the dissipation inequality (5.15) should be replaced by $C$. 


\section{Proof of Main Theorem}

Our proof of the Main Theorem (Theorem 6.1) closely follows that of the Formal Theorem (Theorem 4.1). That proof has six steps:

(1) showing that limiting fluctuations are infinitesimal Maxwellians,

(2) establishing the incompressibility and Boussinesq relations (1.1),

(3) evaluating the limit for moments of the form $\left\langle\mathcal{L} \xi g_{\epsilon}\right\rangle / \epsilon$ for every $\xi \in \operatorname{Dom}(\mathcal{L}) \cap \operatorname{Null}(\mathcal{L})^{\perp}$,

(4) determining the limit of the flux terms in (4.6) that involve $A$ and $B$,

(5) showing that the limiting dynamics is governed by the NavierStokes-Fourier motion and heat equations (1.2),

(6) determining the limit of the deviation of $g_{\epsilon}$ from its infinitesimal Maxwellian.

The analogs of the steps $2-5$ are not easy to realize because DiPernaLions solutions are not known to satisfy most of the conservation laws that were used extensively in the proof of the Formal Theorem. We therefore have to recover these conservation laws in the limit. This is done by taking the velocity moments of the renormalized Boltzmann equation with respect to $v$ and $|v|^{2}$ and showing that the resulting conservation defects vanish as $\epsilon \rightarrow 0$. The most difficult aspect of the proof is to gain enough control of the fluctuations so that in the analogs of steps 3 and 4 we can justify passing to the limit in the nonlinear terms.

In order to clarify the structure the proof, we defer the proofs of many technical details to later sections.

7.1. Fluctuations. Assertion (a) of the Fluctuations Lemma 8.1 will state the family $g_{\epsilon}$ is relatively compact in $w-L_{l o c}^{1}\left(\mathrm{~d} t ; w-L^{1}(\sigma M \mathrm{~d} v \mathrm{~d} x)\right)$. We will show that every limit point of the family $g_{\epsilon}$ is governed by a Leray solution of the Navier-Stokes system.

Consider any convergent subsequence of the family $g_{\epsilon}$, still abusively denoted $g_{\epsilon}$. Let $g$ be the $w-L_{l o c}^{1}\left(\mathrm{~d} t ; w-L^{1}(\sigma M \mathrm{~d} v \mathrm{~d} x)\right)$ limit point of the sequence $g_{\epsilon}$. Assertion (f) of the Fluctuations Lemma 8.1 will state that $g$ is an infinitesimal Maxwellian given by

$$
g=\rho+v \cdot u+\left(\frac{1}{2}|v|^{2}-\frac{D}{2}\right) \theta,
$$

for some $(\rho, u, \theta) \in L^{\infty}\left(\mathrm{d} t ; L^{2}\left(\mathrm{~d} x ; \mathbb{R} \times \mathbb{R}^{D} \times \mathbb{R}\right)\right)$. By the analogs of steps 2 through 5 in the formal derivation, we will show that $(\rho, u, \theta)$ is a Leray solution of the Navier-Stokes-Fourier system (1.1-1.2) with initial data $\left(u^{i n}, \theta^{i n}\right)$. 
7.2. Nonlinear Compactness by Averaging. Key to our proof is the fact the sequence $g_{\epsilon}$ satisfies

$$
\frac{g_{\epsilon}^{2}}{n_{\epsilon}} \text { is relatively compact in } w-L_{l o c}^{1}\left(\mathrm{~d} t ; w-L^{1}(a M \mathrm{~d} v \mathrm{~d} x)\right),
$$

where $n_{\epsilon}=1+\frac{1}{3} \epsilon g_{\epsilon}$. We establish this fact in Section 9 by employing the $L^{1}$ velocity averaging theorem of Golse and Saint-Raymond [24]. They used this averaging theory to prove analogous compactness results while establishing Navier-Stokes-Fourier limits for collision kernels with a Grad cutoff that derive from Maxwell [25] and hard potentials [26].

7.3. Approximate Local Conservation Laws. In order to prove our main theorem we have to pass to the limit in approximate local conservation laws built from the renormalized Boltzmann equation (5.1). We choose to use the normalization of that equation given by

$$
\Gamma(Z)=\frac{Z-1}{1+(Z-1)^{2}}
$$

After dividing by $\epsilon$, equation (5.1) becomes

$$
\epsilon \partial_{t} \tilde{g}_{\epsilon}+v \cdot \nabla_{x} \tilde{g}_{\epsilon}=\frac{1}{\epsilon^{2}} \Gamma^{\prime}\left(G_{\epsilon}\right) \mathcal{Q}\left(G_{\epsilon}, G_{\epsilon}\right),
$$

where $\tilde{g}_{\epsilon}=\Gamma\left(G_{\epsilon}\right) / \epsilon$. By introducing $N_{\epsilon}=1+\epsilon^{2} g_{\epsilon}^{2}$, we can write

$$
\tilde{g}_{\epsilon}=\frac{g_{\epsilon}}{N_{\epsilon}}, \quad \Gamma^{\prime}\left(G_{\epsilon}\right)=\frac{2}{N_{\epsilon}^{2}}-\frac{1}{N_{\epsilon}} .
$$

When the moment of the renormalized Boltzmann equation (7.4) is formally taken with respect to any $\zeta \in \operatorname{span}\left\{1, v_{1}, \cdots, v_{D},|v|^{2}\right\}$, one obtains

$$
\left.\partial_{t}\left\langle\zeta \tilde{g}_{\epsilon}\right\rangle+\frac{1}{\epsilon} \nabla_{x} \cdot\left\langle v \zeta \tilde{g}_{\epsilon}\right\rangle=\frac{1}{\epsilon}\left\langle\zeta \Gamma^{\prime}\left(G_{\epsilon}\right) q_{\epsilon}\right\rangle\right\rangle .
$$

This fails to be a local conservation law because the so-called conservation defect on the right-hand side is generally nonzero.

It can be shown from (5.3) that every DiPerna-Lions solution satisfies (7.6) in the sense that for every $\chi \in C^{1}\left(\mathbb{T}^{D}\right)$ and every $\left[t_{1}, t_{2}\right] \subset[0, \infty)$ it satisfies

$$
\begin{aligned}
\int \chi\left\langle\zeta \tilde{g}_{\epsilon}\left(t_{2}\right)\right\rangle \mathrm{d} x & -\int \chi\left\langle\zeta \tilde{g}_{\epsilon}\left(t_{1}\right)\right\rangle \mathrm{d} x \\
= & \int_{t_{1}}^{t_{2}} \int \frac{1}{\epsilon} \nabla_{x} \chi \cdot\left\langle v \zeta \tilde{g}_{\epsilon}\right\rangle \mathrm{d} x \mathrm{~d} t \\
& +\int_{t_{1}}^{t_{2}} \int \chi \frac{1}{\epsilon}\left\langle\left\langle\zeta \Gamma^{\prime}\left(G_{\epsilon}\right) q_{\epsilon}\right\rangle \mathrm{d} x \mathrm{~d} t\right.
\end{aligned}
$$


This is the sense in which we understand (7.6) is satisfied. Approximate global conservation laws are obtained by setting $\chi=1$ above.

The fact that the conservation defect term on the right-hand side of (7.7) vanishes as $\epsilon \rightarrow 0$ follows from the fact $\chi$ is bounded, the fact $\zeta$ is a collision invariant, and the compactness result (7.2). Specifically, we show that

$$
\frac{1}{\epsilon}\left\langle\left\langle\zeta \Gamma^{\prime}\left(G_{\epsilon}\right) q_{\epsilon}\right\rangle \rightarrow 0 \quad \text { in } L_{l o c}^{1}\left(\mathrm{~d} t ; L^{1}(\mathrm{~d} x)\right) \text { as } \epsilon \rightarrow 0 .\right.
$$

This fact is established by Theorem 10.1, which is stated and proved in Section 10.

7.4. Approximate Dynamical Equations. The difficulty in passing to the limit in (7.6) is that the fluxes are order $1 / \epsilon$. This difficulty is overcome by following the same strategy as in our formal derivation. First, we pass to the limit when $\zeta=v_{i}$ for $i=1, \ldots, D$ or when $\zeta=$ $\left(\frac{1}{2}|v|^{2}-\frac{D+2}{2}\right)$. In other words, we pass to the limit in the approximate motion and heat equations

$$
\partial_{t}\left\langle v \tilde{g}_{\epsilon}\right\rangle+\frac{1}{\epsilon} \nabla_{x} \cdot\left\langle A \tilde{g}_{\epsilon}\right\rangle+\frac{1}{\epsilon} \nabla_{x}\left\langle\frac{1}{D}|v|^{2} \tilde{g}_{\epsilon}\right\rangle=\frac{1}{\epsilon}\left\langle\left\langle v \Gamma^{\prime}\left(G_{\epsilon}\right) q_{\epsilon}\right\rangle\right\rangle,
$$

$$
\partial_{t}\left\langle\left(\frac{1}{2}|v|^{2}-\frac{D+2}{2}\right) \tilde{g}_{\epsilon}\right\rangle+\frac{1}{\epsilon} \nabla_{x} \cdot\left\langle B \tilde{g}_{\epsilon}\right\rangle=\frac{1}{\epsilon}\left\langle\left\langle\left(\frac{1}{2}|v|^{2}-\frac{D+2}{2}\right) \Gamma^{\prime}\left(G_{\epsilon}\right) q_{\epsilon}\right\rangle .\right.
$$

Also as in the formal derivation, the approximate momentum equation (7.9) will be integrated against divergence-free test functions. The last term in its flux will thereby be eliminated, and we only have to pass to the limit in the flux terms above that involve $A$ and $B$ - namely, in the terms

$$
\frac{1}{\epsilon}\left\langle A \tilde{g}_{\epsilon}\right\rangle, \quad \frac{1}{\epsilon}\left\langle B \tilde{g}_{\epsilon}\right\rangle
$$

Recall that $A=\mathcal{L} \widehat{A}$ and $B=\mathcal{L} \widehat{B}$ where $\widehat{A}$ and $\widehat{B}$ are defined by $(4.15)$ and that each entry of $\widehat{A}$ and $\widehat{B}$ is in $L^{p}(a M \mathrm{~d} v)$ for every $p \in[1, \infty)$.

7.4.1. Compactness of the Flux Terms. Let $s \in(1, \infty]$ be from the assumed bound (3.15) on $b$. Let $p=2+\frac{1}{s-1}$, so that $p=2$ when $s=\infty$. Let $\hat{\xi} \in L^{p}(a M \mathrm{~d} v)$ such that $\mathcal{P} \hat{\xi}=0$ and set $\xi=\mathcal{L} \hat{\xi}$. We claim that the sequence of moments

$$
\frac{1}{\epsilon}\left\langle\xi \tilde{g}_{\epsilon}\right\rangle \text { is relatively compact in } w-L_{l o c}^{1}\left(\mathrm{~d} t ; w-L^{1}(\mathrm{~d} x)\right) .
$$


Because each entry of the flux terms (7.11) has this form, it follows that

$$
\begin{aligned}
& \text { the entries of } \frac{1}{\epsilon}\left\langle A \tilde{g}_{\epsilon}\right\rangle \text { and } \frac{1}{\epsilon}\left\langle B \tilde{g}_{\epsilon}\right\rangle \text { are } \\
& \text { relatively compact in } w-L_{l o c}^{1}\left(\mathrm{~d} t ; w-L^{1}(\mathrm{~d} x)\right) \text {. }
\end{aligned}
$$

The claim (7.12) is proved as follows. First, observe that

$$
\left\langle\xi \tilde{g}_{\epsilon}\right\rangle=\left\langle(\mathcal{L} \hat{\xi}) \tilde{g}_{\epsilon}\right\rangle=\left\langle\hat{\xi} \mathcal{L} \tilde{g}_{\epsilon}\right\rangle=\left\langle\left\langle\hat{\xi}\left(\tilde{g}_{\epsilon}+\tilde{g}_{\epsilon 1}-\tilde{g}_{\epsilon}^{\prime}-\tilde{g}_{\epsilon 1}^{\prime}\right)\right\rangle .\right.
$$

Next, introduce the symmetrically normalized collision integrand $\tilde{q}_{\epsilon}$ by

$$
\tilde{q}_{\epsilon}=\frac{q_{\epsilon}}{N_{\epsilon 1}^{\prime} N_{\epsilon}^{\prime} N_{\epsilon 1} N_{\epsilon}}=\frac{1}{\epsilon^{2}} \frac{G_{\epsilon 1}^{\prime} G_{\epsilon}^{\prime}-G_{\epsilon 1} G_{\epsilon}}{N_{\epsilon 1}^{\prime} N_{\epsilon}^{\prime} N_{\epsilon 1} N_{\epsilon}},
$$

and define $T_{\epsilon}$ by

$$
\frac{1}{\epsilon}\left(\tilde{g}_{\epsilon}+\tilde{g}_{\epsilon 1}-\tilde{g}_{\epsilon}^{\prime}-\tilde{g}_{\epsilon 1}^{\prime}\right)=\tilde{g}_{\epsilon 1}^{\prime} \tilde{g}_{\epsilon}^{\prime}-\tilde{g}_{\epsilon 1} \tilde{g}_{\epsilon}-\tilde{q}_{\epsilon}+T_{\epsilon} .
$$

Upon placing (7.16) into the right-hand side of (7.14), the moments (7.12) decompose as

$$
\frac{1}{\epsilon}\left\langle\xi \tilde{g}_{\epsilon}\right\rangle=\left\langle\left\langle\left(\hat{\xi}^{\prime}-\hat{\xi}\right) \tilde{g}_{\epsilon 1} \tilde{g}_{\epsilon}\right\rangle-\left\langle\left\langle\hat{\xi} \tilde{q}_{\epsilon}\right\rangle\right\rangle+\left\langle\left\langle\hat{\xi} T_{\epsilon}\right\rangle\right\rangle .\right.
$$

The first term in this decomposition is quadratic in $\tilde{g}_{\epsilon}$, the second is linear in $\tilde{q}_{\epsilon}$, while the last is a remainder. We control each of these terms separately.

The compactness result (7.2) combined with Lemma 11.2 implies

$$
\left\langle\left(\hat{\xi}^{\prime}-\hat{\xi}\right) \tilde{g}_{\epsilon 1} \tilde{g}_{\epsilon}\right\rangle \quad \text { is relatively compact in } w-L_{l o c}^{1}\left(\mathrm{~d} t ; w-L^{1}(\mathrm{~d} x)\right) \text {. }
$$

This controls the quadratic term in (7.17).

Assertion (c) of the Fluctuations Lemma 8.2 implies that as $\epsilon \rightarrow 0$ one has

$$
\left\langle\hat{\xi} \tilde{q}_{\epsilon}\right\rangle \rightarrow\langle\hat{\xi} A\rangle: \nabla_{x} u+\langle\hat{\xi} B\rangle \cdot \nabla_{x} \theta \quad \text { in } w-L_{l o c}^{2}\left(\mathrm{~d} t ; w-L^{2}(\mathrm{~d} x)\right) .
$$

This controls the linear term in (7.17). In particular, we see that as $\epsilon \rightarrow 0$ one has

$$
\begin{array}{ll}
\left\langle\widehat{A} \tilde{q}_{\epsilon}\right\rangle \rightarrow \nu\left[\nabla_{x} u+\left(\nabla_{x} u\right)^{T}\right] & \text { in } w-L_{l o c}^{2}\left(\mathrm{~d} t ; w-L^{2}\left(\mathrm{~d} x ; \mathbb{R}^{D \times D}\right)\right), \\
\left\langle\widehat{B} \tilde{q}_{\epsilon}\right\rangle \rightarrow \kappa \nabla_{x} \theta & \text { in } w-L_{l o c}^{2}\left(\mathrm{~d} t ; w-L^{2}\left(\mathrm{~d} x ; \mathbb{R}^{D}\right)\right),
\end{array}
$$

where $\nu$ and $\kappa$ are given by (4.18).

The compactness result (7.2) combined with Lemma 11.2 and the Remainder Theorem 12.1 implies that

$$
\left\langle\left\langle\hat{\xi} T_{\epsilon}\right\rangle \rightarrow 0 \text { in } L_{l o c}^{1}\left(\mathrm{~d} t ; L^{1}(\mathrm{~d} x)\right) \text { as } \epsilon \rightarrow 0 .\right.
$$

This controls the last term in (7.17), thereby proving claim (7.12). 
7.4.2. Convergence of the Density Terms. The densities term corresponding to (7.9) and (7.10) are

$$
\Pi\left\langle v \tilde{g}_{\epsilon}\right\rangle \text { and }\left\langle\left(\frac{1}{2}|v|^{2}-\frac{D+2}{2}\right) \tilde{g}_{\epsilon}\right\rangle .
$$

Here $\Pi$ is the Leray projection onto divergence-free vector fields in $L^{2}\left(\mathrm{~d} x ; \mathbb{R}^{D}\right)$. The sequences $(7.20)$ are convergent in $w-L_{l o c}^{2}\left(\mathrm{~d} t ; w-L^{2}(\mathrm{~d} x)\right)$.

We use the Arzela-Ascoli Theorem to establish that these sequences are convergent in $C\left([0, \infty) ; w-L^{2}(\mathrm{~d} x)\right)$. Indeed, it is easy to show that

$$
\left\langle\tilde{g}_{\epsilon}^{2}(t)\right\rangle \leq 3 C^{\text {in }} \text { for every } t \geq 0 .
$$

It then follows from the Cauchy-Schwarz inequality that the sequences (7.20) are equibounded. That they are also equicontinuous follows from the weak forms (7.7) of the approximate motion and heat equations (7.9-7.10) upon noting that the flux terms are relatively compact in $w-L_{l o c}^{1}\left(\mathrm{~d} t ; w-L^{1}(\mathrm{~d} x)\right)$ by (7.13) while the conservation defects vanish by (7.8). The Arzela-Ascoli Theorem then implies that the sequences (7.20) are relatively compact in $C\left([0, \infty) ; w-L^{2}(\mathrm{~d} x)\right)$. Because they are convergent in the weaker topology of $w-L_{l o c}^{2}\left(\mathrm{~d} t ; w-L^{2}(\mathrm{~d} x)\right)$, they must be convergent in $C\left([0, \infty) ; w-L^{2}(\mathrm{~d} x)\right)$. We thereby conclude that as $\epsilon \rightarrow 0$ one has

$$
\begin{aligned}
\Pi\left\langle v \tilde{g}_{\epsilon}\right\rangle & \rightarrow u & & \text { in } C\left([0, \infty) ; w-L^{2}\left(\mathrm{~d} x ; \mathbb{R}^{D}\right)\right), \\
\left\langle\left(\frac{1}{2}|v|^{2}-\frac{D+2}{2}\right) \tilde{g}_{\epsilon}\right\rangle & \rightarrow \frac{D+2}{2} \theta & & \text { in } C\left([0, \infty) ; w-L^{2}(\mathrm{~d} x)\right) .
\end{aligned}
$$

7.5. Recovering the Strong Boussinesq Relation. By assertion (b) of the Fluctuations Lemma, $g$ is of the form of a local infinitesimal Maxwellian (8.10) parametrized by its associated (fluctuation of) velocity field $u$, macroscopic density $\rho$ and temperature $\theta$. Choosing $t_{1}=0, \zeta=|v|^{2}$ and $\chi=1$ in (7.7) shows that

$$
\int\left\langle|v|^{2} \tilde{g}_{\epsilon}\left(t_{2}\right)\right\rangle \mathrm{d} x-\int\left\langle|v|^{2} \tilde{g}_{\epsilon}^{i n}\right\rangle \mathrm{d} x=\int_{0}^{t_{2}} \int \frac{1}{\epsilon}\left\langle|v|^{2} \Gamma^{\prime}\left(G_{\epsilon}\right) q_{\epsilon}\right\rangle \mathrm{d} x \mathrm{~d} t .
$$

By the Conservation Defect Theorem (Proposition 10.1 below), the right-hand side of this equality vanishes with $\epsilon$ uniformly as $t_{2}$ run through any bounded interval of time. Further, the arguments in the last three paragraphs show that the second term in the left-hand side of this equality converges to

$$
\int\left\langle|v|^{2} g^{i n}\right\rangle \mathrm{d} x=0
$$

because of (2.16). Hence, the sequence

$$
\int\left\langle|v|^{2} \tilde{g}_{\epsilon}\right\rangle \mathrm{d} x \rightarrow 0 \quad \text { in } C_{l o c}([0, \infty)) .
$$


Because $\tilde{g}_{\epsilon} \rightarrow g$ in $C\left([0, \infty) ; w-L^{2}(M \mathrm{~d} v \mathrm{~d} x)\right)$,

$$
\int\left\langle|v|^{2} \tilde{g}_{\epsilon}\right\rangle \mathrm{d} x \rightarrow \int\left\langle|v|^{2} g\right\rangle \mathrm{d} x \quad \text { in } C_{l o c}([0, \infty)) .
$$

Hence,

$$
\int\left\langle\frac{1}{D}|v|^{2} g(t)\right\rangle \mathrm{d} x=\int(\rho+\theta)(t) \mathrm{d} x=0
$$

for every $t \geq 0$.

We see from (7.22) that for every $t \geq 0$ the function $(\rho+\theta)(\cdot, t)$ is an element of $L^{2}(\mathrm{~d} x)$ that is orthogonal to the constants. On the other hand, by (8.15), this function also satisfies

$$
\nabla_{x}(\rho+\theta)=0 \text {. }
$$

Then, a classical argument based on Fourier series shows that

$$
\rho+\theta=0 \text { for almost every }(x, t) \in \mathbb{T}^{D} \times[0, \infty) .
$$

By assertion (f) of the Fluctuations Lemma 8.1, this implies that $g$ is in fact of the form (6.6) as stated by the Main Theorem 6.1.

7.6. Convergence of the Quadratic Terms. Now, we concentrate on the passage to the limit in the quadratic term in the decomposition (7.17). This term has the equivalent forms

$$
\left\langle\left(\hat{\xi}^{\prime}-\hat{\xi}\right) \tilde{g}_{\epsilon 1} \tilde{g}_{\epsilon}\right\rangle=\left\langle\hat{\xi}\left(\tilde{g}_{\epsilon 1}^{\prime} \tilde{g}_{\epsilon}^{\prime}-\tilde{g}_{\epsilon 1} \tilde{g}_{\epsilon}\right)\right\rangle=\left\langle\hat{\xi} \mathcal{Q}\left(\tilde{g}_{\epsilon}, \tilde{g}_{\epsilon}\right)\right\rangle .
$$

This passage to the limit is the most difficult part of the proof.

7.6.1. Approximation by Infinitesimal Maxwellians. We decompose $\tilde{g}_{\epsilon}$ into its infinitesimal Maxwellian $\mathcal{P} \tilde{g}_{\epsilon}$ and its deviation $\mathcal{P}^{\perp} \tilde{g}_{\epsilon}$ as

$$
\tilde{g}_{\epsilon}=\mathcal{P} \tilde{g}_{\epsilon}+\mathcal{P}^{\perp} \tilde{g}_{\epsilon} .
$$

We now show that $\tilde{g}_{\epsilon}$ is approximated by its infinitesimal Maxwellian. Specifically, we claim that

$$
\mathcal{P}^{\perp} \tilde{g}_{\epsilon} \rightarrow 0 \quad \text { in } L_{l o c}^{2}\left(\mathrm{~d} t ; L^{2}(a M \mathrm{~d} v \mathrm{~d} x)\right) .
$$

Indeed, multiply (7.16) by $\epsilon \tilde{g}_{\epsilon}$ and integrate to obtain

$$
\left\langle\tilde{g}_{\epsilon} \mathcal{L} \tilde{g}_{\epsilon}\right\rangle=\epsilon\left\langle\left\langle\tilde{g}_{\epsilon}\left(\tilde{g}_{\epsilon 1}^{\prime} \tilde{g}_{\epsilon}^{\prime}-\tilde{g}_{\epsilon 1} \tilde{g}_{\epsilon}\right)\right\rangle-\epsilon\left\langle\tilde{g}_{\epsilon} \tilde{q}_{\epsilon}\right\rangle+\epsilon\left\langle\left\langle\tilde{g}_{\epsilon} T_{\epsilon}\right\rangle .\right.\right.
$$

We treat each term on the right-hand side above separately and show they each vanish as $\epsilon \rightarrow 0$.

First, because $\left|\tilde{g}_{\epsilon 1} \tilde{g}_{\epsilon}\right| \leq \tilde{g}_{\epsilon 1}^{2}+\tilde{g}_{\epsilon}^{2}$, the compactness result (7.2) implies $\tilde{g}_{\epsilon 1} \tilde{g}_{\epsilon}$ and $\tilde{g}_{\epsilon 1}^{\prime} \tilde{g}_{\epsilon}^{\prime}$ are relatively compact in $w-L_{l o c}^{1}\left(\mathrm{~d} t ; w-L^{1}(\mathrm{~d} \mu \mathrm{d} x)\right)$. The 
fact $\epsilon \tilde{g}_{\epsilon}$ is bounded and vanishes almost everywhere as $\epsilon \rightarrow 0$ then implies

$$
\lim _{\epsilon \rightarrow 0} \epsilon\left\langle\left\langle\tilde{g}_{\epsilon}\left(\tilde{g}_{\epsilon 1}^{\prime} \tilde{g}_{\epsilon}^{\prime}-\tilde{g}_{\epsilon 1} \tilde{g}_{\epsilon}\right)\right\rangle=0 \quad \text { in } L_{l o c}^{1}\left(\mathrm{~d} t ; L^{1}(\mathrm{~d} x)\right) .\right.
$$

Hence, the first term on the right-hand side of (7.27) vanishes as $\epsilon \rightarrow 0$.

Next, assertions (d), (e) and (g) of the First Fluctuations Lemma 8.1 imply that $\tilde{g}_{\epsilon}=O(|\log (\epsilon)|)$ in $L^{\infty}\left(\mathrm{d} t ; L^{2}(a M \mathrm{~d} v \mathrm{~d} x)\right)$ and that $\tilde{q}_{\epsilon}$ is bounded in $L_{l o c}^{2}\left(\mathrm{~d} t ; L^{2}(\mathrm{~d} \mu \mathrm{d} x)\right)$. It follows that

$$
\lim _{\epsilon \rightarrow 0} \epsilon\left\langle\left\langle\tilde{g}_{\epsilon} \tilde{q}_{\epsilon}\right\rangle=0 \quad \text { in } L_{l o c}^{1}\left(\mathrm{~d} t ; L^{1}(\mathrm{~d} x)\right) .\right.
$$

The second term on the right-hand side of (7.27) thereby vanishes as $\epsilon \rightarrow 0$.

Finally, because $\epsilon \tilde{g}_{\epsilon}$ is bounded, the compactness result (7.2) combined with Lemma 11.2 and the Remainder Theorem 12.1 implies that

$$
\lim _{\epsilon \rightarrow 0} \epsilon\left\langle\left\langle\tilde{g}_{\epsilon} T_{\epsilon}\right\rangle=0 \quad \text { in } L_{l o c}^{1}\left(\mathrm{~d} t ; L^{1}(\mathrm{~d} x)\right) .\right.
$$

The third term on the right-hand side of (7.27) thereby vanishes as $\epsilon \rightarrow 0$.

Upon combining (7.28-7.30) with (7.27), we conclude that

$$
\lim _{\epsilon \rightarrow 0}\left\langle\tilde{g}_{\epsilon} \mathcal{L} \tilde{g}_{\epsilon}\right\rangle=0 \quad \text { in } L_{l o c}^{1}\left(\mathrm{~d} t ; L^{1}(\mathrm{~d} x)\right) .
$$

The coercivity bound (3.36) then immediately implies

$$
\lim _{\epsilon \rightarrow 0}\left\langle a\left(\mathcal{P}^{\perp} \tilde{g}_{\epsilon}\right)^{2}\right\rangle=0 \quad \text { in } L_{l o c}^{1}\left(\mathrm{~d} t ; L^{1}(\mathrm{~d} x)\right),
$$

which establishes claim (7.26).

7.6.2. Quadratic Approximation by Infinitesimal Maxwellians. When decomposition (7.25) is placed into the quadratic term (7.24), it yields

$$
\begin{aligned}
\left\langle\hat{\xi} \mathcal{Q}\left(\tilde{g}_{\epsilon}, \tilde{g}_{\epsilon}\right)\right\rangle=\left\langle\hat{\xi} \mathcal{Q}\left(\mathcal{P} \tilde{g}_{\epsilon}, \mathcal{P} \tilde{g}_{\epsilon}\right)\right\rangle & +2\left\langle\hat{\xi} \mathcal{Q}\left(\mathcal{P} \tilde{g}_{\epsilon}, \mathcal{P}^{\perp} \tilde{g}_{\epsilon}\right)\right\rangle \\
& +\left\langle\hat{\xi} \mathcal{Q}\left(\mathcal{P}^{\perp} \tilde{g}_{\epsilon}, \mathcal{P}^{\perp} \tilde{g}_{\epsilon}\right)\right\rangle .
\end{aligned}
$$

Here we show the last two terms above vanish as $\epsilon \rightarrow 0$.

Recall that the collision kernel $b$ satisfies assumption (3.15) for some $C_{b}<\infty$ and $s \in(1, \infty]$. Let $p=2+\frac{1}{s-1}$, so $p=2$ when $s=\infty$. Lemma 11.1 of Section 11 then combines with (7.24) to yield the basic quadratic estimate

$$
|\langle\hat{\xi} \mathcal{Q}(\tilde{g}, \tilde{h})\rangle| \leq 2 C_{b}^{\frac{1}{p^{*}}}\left\langle a|\hat{\xi}|^{p}\right\rangle^{\frac{1}{p}}\left\langle a \tilde{g}^{2}\right\rangle^{\frac{1}{2}}\left\langle a \tilde{h}^{2}\right\rangle^{\frac{1}{2}},
$$

where $\frac{1}{p}+\frac{1}{p^{*}}=1$ and $\tilde{g}, \tilde{h} \in L^{2}(a M \mathrm{~d} v)$. 
It follows from (7.26) and the basic estimate (7.32) that

$$
\left.\begin{array}{rl}
\left\langle\hat{\xi} \mathcal{Q}\left(\mathcal{P} \tilde{g}_{\epsilon}, \mathcal{P}^{\perp} \tilde{g}_{\epsilon}\right)\right\rangle & \rightarrow 0 \\
\left\langle\hat{\xi} \mathcal{Q}\left(\mathcal{P}^{\perp} \tilde{g}_{\epsilon}, \mathcal{P}^{\perp} \tilde{g}_{\epsilon}\right)\right\rangle & \rightarrow 0
\end{array}\right\} \quad \text { in } L_{l o c}^{1}\left(\mathrm{~d} t ; L^{1}(\mathrm{~d} x)\right) .
$$

So all that remains to be done is to pass to the limit in the first term on the right-hand side of $(7.31)$ — the one that is quadratic in $\mathcal{P} \tilde{g}_{\epsilon}$.

7.6.3. Passing to the Limit. The infinitesimal Maxwellian $\mathcal{P} \tilde{g}_{\epsilon}$ has the form

$$
\mathcal{P} \tilde{g}_{\epsilon}=\tilde{\rho}_{\epsilon}+v \cdot \tilde{u}_{\epsilon}+\left(\frac{1}{2}|v|^{2}-\frac{D}{2}\right) \tilde{\theta}_{\epsilon},
$$

where $\tilde{\rho}_{\epsilon}, \tilde{u}_{\epsilon}$, and $\tilde{\theta}_{\epsilon}$ are defined by

$$
\tilde{\rho}_{\epsilon}=\left\langle\tilde{g}_{\epsilon}\right\rangle, \quad \tilde{u}_{\epsilon}=\left\langle v \tilde{g}_{\epsilon}\right\rangle, \quad \tilde{\theta}_{\epsilon}=\left\langle\left(\frac{1}{D}|v|^{2}-1\right) \tilde{g}_{\epsilon}\right\rangle .
$$

Because $\mathcal{P} \tilde{g}_{\epsilon}$ is an infinitesimal Maxwellian, we can use the identity (4.12) to express the first term on the right-hand side of (7.31) as

$$
\begin{aligned}
\left\langle\hat{\xi} \mathcal{Q}\left(\mathcal{P} \tilde{g}_{\epsilon}, \mathcal{P} \tilde{g}_{\epsilon}\right)\right\rangle & =\frac{1}{2}\left\langle\xi \mathcal{P}^{\perp}\left(\mathcal{P} \tilde{g}_{\epsilon}\right)^{2}\right\rangle \\
& =\frac{1}{2}\langle\xi A\rangle:\left(\tilde{u}_{\epsilon} \otimes \tilde{u}_{\epsilon}\right)+\langle\xi B\rangle \cdot \tilde{u}_{\epsilon} \tilde{\theta}_{\epsilon}+\frac{1}{2}\langle\xi C\rangle \tilde{\theta}_{\epsilon}^{2},
\end{aligned}
$$

where $C$ is defined by (4.14). We thereby have reduced the problem to passing to the limit in the terms

$$
\tilde{u}_{\epsilon} \otimes \tilde{u}_{\epsilon}, \quad \tilde{u}_{\epsilon} \tilde{\theta}_{\epsilon}, \quad \tilde{\theta}_{\epsilon}^{2} .
$$

We are unable to pass to the limit in the above terms in full generality. Rather, the Quadratic Limit Theorem 13.1 of Section 13 yields that

$$
\left.\begin{array}{rl}
\lim _{\epsilon \rightarrow 0} \Pi \nabla_{x} \cdot\left(\tilde{u}_{\epsilon} \otimes \tilde{u}_{\epsilon}\right) & =\Pi \nabla_{x} \cdot(u \otimes u) \\
\lim _{\epsilon \rightarrow 0} \nabla_{x} \cdot\left(\tilde{\theta}_{\epsilon} \tilde{u}_{\epsilon}\right) & =\nabla_{x} \cdot(\theta u)
\end{array}\right\} \quad \text { in } w-L_{l o c}^{1}\left(\mathrm{~d} t ; \mathcal{D}^{\prime}\left(\mathbb{T}^{D}\right)\right),
$$

where $\Pi$ is the Leray projection onto divergence-free vector fields in $L^{2}\left(\mathrm{~d} x ; \mathbb{R}^{D}\right)$. It follows that

$$
\left.\begin{array}{l}
\lim _{\epsilon \rightarrow 0} \Pi \nabla_{x} \cdot\left\langle\widehat{A} \mathcal{Q}\left(\mathcal{P} \tilde{g}_{\epsilon}, \mathcal{P} \tilde{g}_{\epsilon}\right)\right\rangle=\Pi \nabla_{x} \cdot(u \otimes u) \\
\lim _{\epsilon \rightarrow 0} \nabla_{x} \cdot\left\langle\widehat{B} \mathcal{Q}\left(\mathcal{P} \tilde{g}_{\epsilon}, \mathcal{P} \tilde{g}_{\epsilon}\right)\right\rangle=\frac{D+2}{2} \nabla_{x} \cdot(\theta u)
\end{array}\right\} \quad \text { in } w-L_{l o c}^{1}\left(\mathrm{~d} t ; \mathcal{D}^{\prime}\left(\mathbb{T}^{D}\right)\right)
$$

We thereby obtain the limiting fluxes for the Navier-Stokes-Fourier motion and heat equations. 


\section{Control of the Fluctuations}

In order to establish fluid dynamical equations from the Boltzmann equation for regimes near an absolute Maxwellian $M$, one must control the fluctuations of the relative kinetic density $G$ about 1 with the DiPerna-Lions entropy inequality (5.9) and with the weak form of the Boltzmann equation (5.3). In this section we collect some such estimates from [3] and [19] that help in this task.

8.1. Controls from the Entropy Inequality. From (6.2) of the Definition 6.1 of entropic convergence, it follows that $G_{\epsilon}^{i n}$ satisfies the entropy bound

$$
H\left(G_{\epsilon}^{i n}\right) \leq C^{i n} \epsilon^{2}, \quad \text { for some } C^{i n}<\infty .
$$

The DiPerna-Lions entropy inequality (5.9) then implies that the family $G_{\epsilon}$ of DiPerna-Lions renormalized solutions satisfies the bound

$$
H\left(G_{\epsilon}(t)\right)+\frac{1}{\epsilon^{2}} \int_{0}^{t} R\left(G_{\epsilon}(s)\right) \mathrm{d} s \leq H\left(G_{\epsilon}^{i n}\right) \leq C^{i n} \epsilon^{2} .
$$

In this subsection we give controls on the fluctuations that follow only from the fact that $G_{\epsilon}$ satisfies (8.2).

The relative entropy functional $H$ given by (2.19) has an integrand that is a nonnegative strictly convex function of $G$ with a minimum value of 0 at $G=1$. It thereby provides a natural measure of the proximity of $G$ to that equilibrium. For the families of fluctuations $g_{\epsilon}^{i n}$ and $g_{\epsilon}$ given by (6.4) and (6.1), one easily sees that $H$ asymptotically behaves like half the square of the $L^{2}(M \mathrm{~d} v)$-norm of these fluctuations as $\epsilon \rightarrow 0$. Hence, the bound (8.2) is consistent with these fluctuations being order one.

Just as the relative entropy $H$ controls the fluctuations $g_{\epsilon}$, the dissipation rate $R$ given by (2.20) controls the scaled collision integrands defined by

$$
q_{\epsilon}=\frac{G_{\epsilon 1}^{\prime} G_{\epsilon}^{\prime}-G_{\epsilon 1} G_{\epsilon}}{\epsilon^{2}}
$$

Once again, the bound (8.2) is consistent with these scaled integrands being order one.

The following shows that more is true.

Lemma 8.1. (First Fluctuations Lemma) Let $b$ be a collision kernel that satisfies conditions (3.7), (3.9), and (3.15).

Let $G_{\epsilon} \geq 0$ be a family of functions in $C\left([0, \infty) ; w-L^{1}(M \mathrm{~d} v \mathrm{~d} x)\right.$ that satisfies the bound (8.2) with $G_{\epsilon}^{i n}=G_{\epsilon}(0)$ for some $C^{\text {in }}<\infty$. Let $g_{\epsilon}^{\text {in }}$, 
$g_{\epsilon}$, and $q_{\epsilon}$ be given by (6.4), (6.1) and (8.3). Define $n_{\epsilon}$ by

$$
n_{\epsilon}=\frac{2}{3}+\frac{1}{3} G_{\epsilon}=1+\frac{1}{3} \epsilon g_{\epsilon} .
$$

Then, adopting the notation $\sigma=1+|v|^{2}$, we have the following.

(a) The family $g_{\epsilon}$ is bounded in $L^{\infty}\left(\mathrm{d} t ; L^{1}(\sigma M \mathrm{~d} v \mathrm{~d} x)\right)$, relatively compact in $w-L_{l o c}^{1}\left(\mathrm{~d} t ; w-L^{1}(\sigma M \mathrm{~d} v \mathrm{~d} x)\right)$, and relatively compact in $w-L^{1}(\sigma M \mathrm{~d} v \mathrm{~d} x)$ pointwise over $t \geq 0$.

(b) The family $q_{\epsilon} / \sqrt{n_{\epsilon}}$ is relatively compact in $w-L_{l o c}^{1}\left(\mathrm{~d} t ; w-L^{1}(\sigma \mathrm{d} \mu \mathrm{d} x)\right)$.

(c) If $g^{\text {in }}$ is a $w-L^{1}(\sigma M \mathrm{~d} v \mathrm{~d} x)$ limit point of the family $g_{\epsilon}^{\text {in }}$ as $\epsilon \rightarrow 0$ then $g^{\text {in }} \in L^{2}(M \mathrm{~d} v \mathrm{~d} x)$ and one has

$$
\frac{1}{2} \int_{\mathbb{T}^{D}}\left\langle g^{i n 2}\right\rangle \mathrm{d} x \leq \liminf _{\epsilon \rightarrow 0} \frac{1}{\epsilon^{2}} H\left(G_{\epsilon}^{i n}\right) \leq C^{i n} .
$$

(d) If $g$ is a $w-L_{l o c}^{1}\left(\mathrm{~d} t ; w-L^{1}(\sigma M \mathrm{~d} v \mathrm{~d} x)\right)$ limit point of the family $g_{\epsilon}$ and $q$ is jointly a $w-L_{l o c}^{1}\left(\mathrm{~d} t ; w-L^{1}(\sigma \mathrm{d} \mu \mathrm{d} x)\right)$ limit point of the family $q_{\epsilon} / \sqrt{n_{\epsilon}}$ as $\epsilon \rightarrow 0$ then $g \in L^{\infty}\left(\mathrm{d} t ; L^{2}(M \mathrm{~d} v \mathrm{~d} x)\right), q \in$ $L^{2}(\mathrm{~d} \mu \mathrm{d} x \mathrm{~d} t)$, and $q$ inherits the symmetries of $q_{\epsilon}$. Moreover, for almost every $t \geq 0$ one has

$$
\frac{1}{2} \int_{\mathbb{T}^{D}}\left\langle g(t)^{2}\right\rangle \mathrm{d} x \leq \liminf _{\epsilon \rightarrow 0} \frac{1}{\epsilon^{2}} H\left(G_{\epsilon}(t)\right) \leq C^{i n},
$$

while for every $t \geq 0$ one has

$$
\frac{1}{4} \int_{0}^{t} \int_{\mathbb{T}^{D}}\left\langle\left\langle q(s)^{2}\right\rangle \mathrm{d} x \mathrm{~d} s \leq \liminf _{\epsilon \rightarrow 0} \frac{1}{\epsilon^{4}} \int_{0}^{t} R\left(G_{\epsilon}(s)\right) \mathrm{d} s \leq C^{i n} .\right.
$$

(e) The family $g_{\epsilon}$ satisfies the nonlinear estimates

$$
\begin{gathered}
\frac{g_{\epsilon}^{2}}{n_{\epsilon}}(t) \quad \text { is bounded in } L^{1}(M \mathrm{~d} v \mathrm{~d} x) \text { uniformly over } t \geq 0 \\
\sigma \frac{g_{\epsilon}^{2}}{n_{\epsilon}}=O(|\log (\epsilon)|) \quad \text { in } L^{\infty}\left(\mathrm{d} t ; L^{1}(M \mathrm{~d} v \mathrm{~d} x)\right) \text { as } \epsilon \rightarrow 0
\end{gathered}
$$

(f) Let $g$ be as in (d) above. Then $g$ has the form of an infinitesimal Maxwellian,

$$
g=\rho+v \cdot u+\left(\frac{1}{2}|v|^{2}-\frac{D}{2}\right) \theta
$$

for some $(\rho, u, \theta) \in L^{\infty}\left(\mathrm{d} t ; L^{2}\left(\mathrm{~d} x ; \mathbb{R} \times \mathbb{R}^{D} \times \mathbb{R}\right)\right.$. 
(g) The family $q_{\epsilon}$ satisfies the nonlinear estimates

$$
\begin{gathered}
\frac{q_{\epsilon}^{2}}{n_{\epsilon 1}^{\prime} n_{\epsilon}^{\prime} n_{\epsilon 1} n_{\epsilon}} \quad \text { is bounded in } L^{1}(\mathrm{~d} \mu \mathrm{d} x \mathrm{~d} t), \\
\sigma \frac{q_{\epsilon}^{2}}{n_{\epsilon 1}^{\prime} n_{\epsilon}^{\prime} n_{\epsilon 1} n_{\epsilon}}=O(|\log (\epsilon)|) \quad \text { in } L_{l o c}^{1}\left(\mathrm{~d} t ; L^{1}(\mathrm{~d} \mu \mathrm{d} x)\right) \text { as } \epsilon \rightarrow 0 .
\end{gathered}
$$

Assertion (a) is essentially Proposition 3.1 (1) of [3]. Assertion (b) is Proposition 3.4 (1) of [3]. Assertion (c) is essentially contained in Proposition 3.1 (2) of [3]. Assertion (d) consolidates Proposition 3.1 (2) and Proposition 3.4 (2) of [3]. Assertion (e) consolidates Proposition 3.2 (3) and Proposition 3.3 of [3]. Estimate (8.9) is the key nonlinear estimate from [3]. Assertion (f) is Proposition 3.8 of [3]. It is a consequence of assertions (a), (b) and (e). Assertion (g) consolidates Lemmas 10.1 and 9.3 of [19].

8.2. Controls from the Boltzmann Equation. The following are amplifications of Proposition 8.1 that result because $G_{\epsilon}$ are renormalized solutions of the Boltzmann equation.

Lemma 8.2. (Second Fluctuations Lemma) Let $b$ be a collision kernel that satisfies assumptions (3.7), (3.9), (3.15), and (3.26).

Let $G_{\epsilon} \geq 0$ be a family of renormalized solutions of the scaled initialvalue problem (2.7) with initial data $G_{\epsilon}^{\text {in }}$ that satisfy the entropy bound (8.1) for some $C^{i n}<\infty$. Let $g_{\epsilon}$ and $q_{\epsilon}$ be the corresponding fluctuations (6.1) and scaled collision integrands (8.3). Let $n_{\epsilon}$ be given by (8.4).

(a) For every $\left[t_{1}, t_{2}\right] \subset[0, \infty)$ and every $\xi=\xi(v)$ such that $|\xi| / \sigma$ is bounded, the family $g_{\epsilon}$, uniformly in $\epsilon$, satisfies

$$
\int_{t_{1}}^{t_{2}} \int_{\mathbb{T}^{D}}\left|\left\langle\xi \frac{g_{\epsilon}}{n_{\epsilon}}\right\rangle(x-y, t)-\left\langle\xi \frac{g_{\epsilon}}{n_{\epsilon}}\right\rangle(x, t)\right| \mathrm{d} x \mathrm{~d} t \rightarrow 0 \quad \text { as } y \rightarrow 0 \text {. }
$$

(b) Let $g$ be a $w-L_{l o c}^{1}\left(\mathrm{~d} t ; w-L^{1}(\sigma M \mathrm{~d} v \mathrm{~d} x)\right)$ limit point of the family $g_{\epsilon}$ and $q$ be jointly a $w-L_{l o c}^{1}\left(\mathrm{~d} t ; w-L^{1}(\sigma \mathrm{d} \mu \mathrm{d} x)\right)$ limit point of the family $q_{\epsilon} / \sqrt{n_{\epsilon}}$ as $\epsilon \rightarrow 0$. Then $g \in L^{\infty}\left(\mathrm{d} t ; L^{2}(M \mathrm{~d} v \mathrm{~d} x)\right)$ and $q \in L^{2}(\mathrm{~d} \mu \mathrm{d} x \mathrm{~d} t)$ satisfy

$$
v \cdot \nabla_{x} g=\iint_{\mathbb{S}^{D-1} \times \mathbb{R}^{D}} q b\left(\omega, v_{1}-v\right) \mathrm{d} \omega M_{1} \mathrm{~d} v_{1} ;
$$

(c) Let $g$ and $q$ be as in (b). Then $g$ is an infinitesimal Maxwellian of the form (8.10) where $(\rho, u, \theta) \in L^{\infty}\left(\mathrm{d} t ; L^{2}(\mathrm{~d} x)\right) \cap L^{2}\left(\mathrm{~d} t ; H^{1}(\mathrm{~d} x)\right)$ satisfies

$$
\nabla_{x} \cdot u=0, \quad \nabla_{x}(\rho+\theta)=0,
$$


while $q$ satisfies the relations

$$
\langle\widehat{A} q\rangle=\nu\left(\nabla_{x} u+\left(\nabla_{x} u\right)^{T}\right), \quad\langle\widehat{B} q\rangle=\kappa \nabla_{x} \theta,
$$

and the inequality

$$
\int_{0}^{t} \int_{\mathbb{T}^{D}} \nu\left|\nabla_{x} u\right|^{2}+\kappa\left|\nabla_{x} \theta\right|^{2} \mathrm{~d} x \mathrm{~d} s \leq \frac{1}{4} \int_{0}^{t} \int_{\mathbb{T}^{D}}\left\langle\left\langle q^{2}\right\rangle \mathrm{d} x \mathrm{~d} s\right.
$$

for every $t \geq 0$, where $\widehat{A}$ and $\widehat{B}$ are given by (4.15) while $\nu$ and $\kappa$ are given by (4.18).

Assertion (a) follows from assertions (a) and (b) of the First Fluctuations Lemma (Lemma 8.1) by an application of the $L^{1}$-velocity averaging result of [20]. Assertion (b) is essentially Proposition 4.1 of [3]. Assertion (c) strengthens assertion (f) of Lemma 8.1. It consolidates Propositions 4.2, 4.3, and 4.6 of [3]. The proofs of the last two assertions each rest on the key nonlinear estimate (8.9). 


\section{Compactness from Averaging}

In this section, we want to prove the following Proposition

Proposition 9.1. Under the hypotheses of the main theorem 6.1, we have

$$
(1+a(v)) \frac{g_{\epsilon}^{2}}{n_{\epsilon}} \quad \text { is relatively compact in } w-L_{l o c}^{1}\left(\mathrm{~d} t ; w-L^{1}(M \mathrm{~d} v \mathrm{~d} x)\right) .
$$

Proof of Proposition 9.1. The Proposition is a simple consequence of the following two lemmas.

Lemma 9.1. Under the hypotheses of the main theorem, for all $R>0$, we have

(9.2) $1_{\{|v|<R\}} \frac{g_{\epsilon}^{2}}{n_{\epsilon}} \quad$ is relatively compact in $w-L_{l o c}^{1}\left(\mathrm{~d} t ; w-L^{1}(M \mathrm{~d} v \mathrm{~d} x)\right)$.

Lemma 9.2. Under the hypotheses of the main theorem,

$$
\lim _{R \rightarrow \infty} \sup _{\epsilon}\left\|1_{\{|v|>R\}} \frac{g_{\epsilon}^{2}}{n_{\epsilon}}\right\|_{L^{1}\left((0, T) ; L^{1}((1+a(v)) M \mathrm{~d} v \mathrm{~d} x)\right)}=0 .
$$

Now, we concentrate on the proof of the two lemmas. We start with lemma 9.2 .

Proof of Lemma 9.2. To explain the idea, we will start with the case we assume that $\bar{b}_{H}(v)$ is bounded. We recall the definition of $a_{H}(v)$ and $\bar{b}_{H}(v)$,

$$
a_{H}(v)=\int_{\mathbb{R}^{D}} \bar{b}_{H}\left(v_{1}-v\right) M_{1} \mathrm{~d} v_{1}=\iint_{\mathbb{S}^{D-1} \times \mathbb{R}^{D}} b_{H}\left(\omega, v_{1}-v\right) \mathrm{d} \omega M_{1} \mathrm{~d} v_{1},
$$

where

$$
\begin{array}{ll}
b_{H}(\omega, v)=b(\omega, v), & \text { for }|v|>V_{0}, \\
b_{H}(\omega, v)=\min \{b(\omega, v), C\}, & \text { for }|v| \leq V_{0} .
\end{array}
$$

Since $\bar{b}_{H}(v)$ is bounded, $a_{H}(v)$ is also bounded. Moreover, because $b$ and $b_{H}$ differ only on the set $|v|<V_{0}$, we deduce that $a_{H}(v)$ also satisfies the same bound from below as $a(v)$, namely that $a_{H}(v) \geq C_{a}(1+|v|)^{\alpha}$ for some $\alpha \in \mathcal{R}$. Actually, for this section we only need a weaker lower bound $a_{H}(v) \geq c M(v)^{\alpha}$ for some $0<\alpha<1$. We define $\mathcal{L}_{H}$ and $\mathcal{Q}_{H}$ by replacing $b$ by $b_{H}$ in the definition of $\mathcal{L}$ and $\mathcal{Q}$. We also denote $\sqrt{G_{\epsilon}}=1+\epsilon \phi_{\epsilon}$. We notice that $\phi_{\epsilon}^{2}$ and $\frac{g_{\epsilon}^{2}}{n_{\epsilon}}$ behave in the same way for 
small and for large values of $\phi_{\epsilon}$, so it is enough to prove the estimates for $\phi_{\epsilon}^{2}$ instead of $\frac{g_{\epsilon}^{2}}{n_{\epsilon}}$. We have

$$
\mathcal{L}_{H} \phi_{\epsilon}=\epsilon \mathcal{Q}_{H}\left(\phi_{\epsilon}, \phi_{\epsilon}\right)-\frac{1}{\epsilon} \mathcal{Q}_{H}\left(\sqrt{G_{\epsilon}}, \sqrt{G_{\epsilon}}\right),
$$

and for almost every $x$ and $t$, we have

$$
\begin{array}{rl}
\left\|\mathcal{P}^{\perp} \phi_{\epsilon}\right\|_{L^{2}\left(a_{H}(v) M \mathrm{~d} v\right) \leq}^{2} & C\left\langle\left(\mathcal{P}^{\perp} \phi_{\epsilon}\right) \mathcal{L}_{H} \phi_{\epsilon}\right\rangle \\
\leq & C \int \epsilon \mathcal{Q}_{H}\left(\phi_{\epsilon}, \phi_{\epsilon}\right) \mathcal{P}^{\perp} \phi_{\epsilon} M \mathrm{~d} v \\
& -\int \frac{1}{\epsilon} \mathcal{Q}_{H}\left(\sqrt{G_{\epsilon}}, \sqrt{G_{\epsilon}}\right) \mathcal{P}^{\perp} \phi_{\epsilon} M \mathrm{~d} v
\end{array}
$$

since $\mathcal{L}_{H}$ satisfies the coercivity property (3.34). Now, we can estimate each term in the following way

$$
\begin{aligned}
\int \mathcal{Q}_{H}\left(\sqrt{G_{\epsilon}}, \sqrt{G_{\epsilon}}\right) \mathcal{P}^{\perp} \phi_{\epsilon} M \mathrm{~d} v \leq & \left(\iiint\left(\mathcal{P}^{\perp} \phi_{\epsilon}\right)^{2} \mathrm{~d} \mu_{H}\right)^{\frac{1}{2}} \\
& \left(\iiint\left(\sqrt{G_{\epsilon 1}^{\prime} G_{\epsilon}^{\prime}}-\sqrt{G_{\epsilon 1} G_{\epsilon}}\right)^{2} \mathrm{~d} \mu_{H}\right)^{\frac{1}{2}},
\end{aligned}
$$

where $\mathrm{d} \mu_{H}=b_{H}\left(\omega, v_{1}-v\right) \mathrm{d} \omega M_{1} \mathrm{~d} v_{1} M \mathrm{~d} v$ and we can see that the second term in the right-hand side is controlled by the entropy dissipation. Recall that $(\sqrt{x}-\sqrt{y})^{2} \leq \frac{1}{4}(x-y) \log (x / y)$.

Moreover, using the assumption that $\bar{b}_{H}$ is bounded, we have

$$
\begin{aligned}
\int & \mathcal{Q}_{H}\left(\phi_{\epsilon}, \phi_{\epsilon}\right) \mathcal{P}^{\perp} \phi_{\epsilon} M \mathrm{~d} v \\
& \leq\left(\iiint\left(\mathcal{P}^{\perp} \phi_{\epsilon}\right)^{2} \mathrm{~d} \mu_{H}\right)^{1 / 2}\left(\iiint\left(\phi_{\epsilon 1}^{\prime} \phi_{\epsilon}^{\prime}\right)^{2}+\left(\phi_{\epsilon 1} \phi_{\epsilon}\right)^{2} \mathrm{~d} \mu_{H}\right)^{1 / 2} \\
& \leq C\left(\int\left(\mathcal{P}^{\perp} \phi_{\epsilon}\right)^{2} a_{H}(v) M \mathrm{~d} v\right)^{1 / 2}\left(\iint\left(\phi_{\epsilon 1} \phi_{\epsilon}\right)^{2} \bar{b}_{H}\left(v_{1}-v\right) M_{1} \mathrm{~d} v_{1} M \mathrm{~d} v\right)^{1 / 2} \\
& \leq\left\|\phi_{\epsilon}\right\|_{L^{2}(M \mathrm{~d} v)}^{2}\left\|\mathcal{P}^{\perp} \phi_{\epsilon}\right\|_{L^{2}\left(a_{H}(v) M \mathrm{~d} v\right)} .
\end{aligned}
$$

Finally, we deduce that

$$
\left\|\mathcal{P}^{\perp} \phi_{\epsilon}\right\|_{L^{2}\left(a_{H}(v) M \mathrm{~d} v\right)} \leq \frac{C}{\epsilon}\left\langle\left\langle\log \left(\frac{G_{1}^{\prime} G^{\prime}}{G_{1} G}\right)\left(G_{1}^{\prime} G^{\prime}-G_{1} G\right)\right\rangle\right\rangle^{1 / 2}+C \epsilon\left\|\phi_{\epsilon}\right\|_{L^{2}(M \mathrm{~d} v)}^{2}
$$

Integrating in $x$ and $t$, we see that $\left\|\mathcal{P}^{\perp} \phi_{\epsilon}\right\|_{L_{l o c}^{1}\left(\mathrm{~d} t ; L^{1}\left(\mathrm{~d} x ; L^{2}\left(a_{H}(v) M \mathrm{~d} v\right)\right)\right)} \leq$ $C \epsilon$. 
Now, take $A>1$. We can write

$$
1_{\{|v|>R\}} \phi_{\epsilon}^{2}=1_{\{|v|>R\}} 1_{\left\{G_{\epsilon}>A\right\}} \phi_{\epsilon}^{2}+1_{\{|v|>R\}} 1_{\left\{G_{\epsilon}<A\right\}}\left(\phi_{\epsilon} \mathcal{P} \phi_{\epsilon}+\phi_{\epsilon} \mathcal{P}^{\perp} \phi_{\epsilon}\right) \text {. }
$$

Upon integrating this over $\mathbb{R}^{D} \times \mathbb{T}^{D} \times(0, T)$, we observe that the first term on the right-hand side can be estimated using the entropy inequality and is of order $\frac{1}{\log A}$ in $L^{\infty}\left(\mathrm{d} t ; L^{1}(M \mathrm{~d} v \mathrm{~d} x)\right)$. The second term is bounded in $L^{\infty}\left(\mathrm{d} t ; L^{1}\left(\mathrm{~d} x ; L^{q}(M \mathrm{~d} v)\right)\right.$ for every $1 \leq q<2$ and hence

$$
\iiint_{(0, T) \times \mathbb{T}^{D} \times \mathbb{R}^{D}} 1_{\{|v|>R\}} 1_{G_{\epsilon}<A}\left|\phi_{\epsilon} \mathcal{P} \phi_{\epsilon}\right| \mathrm{d} t \mathrm{~d} x M \mathrm{~d} v \leq\left(\int_{\{|v|>R\}} M \mathrm{~d} v\right)^{1 / 3}
$$

where we took $q=3 / 2$. The third term is of order $\sqrt{A}$ in $L^{1}\left(\mathrm{~d} x \mathrm{~d} t ; L^{2}\left(a_{H} M \mathrm{~d} v\right)\right)$ since $\left\|1_{G_{\epsilon}<A} \phi_{\epsilon}\right\|_{L^{\infty}} \leq \frac{\sqrt{A}}{\epsilon}$. Hence

$$
\iiint_{\mathbb{R}^{D \times \mathbb{T}^{D} \times(0, T)}} 1_{\{|v|>R\}} 1_{\left\{G_{\epsilon}<A\right\}}\left|\phi_{\epsilon} \mathcal{P}^{\perp} \phi_{\epsilon}\right| M \mathrm{~d} v \mathrm{~d} x \mathrm{~d} t \leq C \sqrt{A}\left(\int_{\{|v|>R\}} M^{1-\alpha} \mathrm{d} v\right)^{1 / 2} .
$$

where we have used that $a_{H}(v) \geq c M^{\alpha}$ for some $0 \leq \alpha<1$. Hence, taking $A$ big and then $R$ big, we see that (9.3) holds. This completes the proof of the lemma for the case when $\bar{b}_{H}(v)$ is bounded.

Next, we consider the general case, namely $\bar{b}_{H}\left(v_{1}-v\right) \leq C(1+$ $\left.a_{H}\left(v_{1}\right)\right)\left(1+a_{H}(v)\right)$ for every $v$ and $v_{1}$. As in the previous case we consider the collision kernel $b_{H}$. It is easy to see that (9.7), and (9.8) still hold. Besides, (9.9) should be replaced by

$$
\begin{aligned}
\int & \mathcal{Q}_{r}\left(\phi_{\epsilon}, \phi_{\epsilon}\right) \mathcal{P}^{\perp} \phi_{\epsilon} M \mathrm{~d} v \\
& \leq C\left(\int\left(\mathcal{P}^{\perp} \phi_{\epsilon}\right)^{2} a_{H}(v) M \mathrm{~d} v\right)^{1 / 2}\left(\iint\left(\phi_{\epsilon} \phi_{\epsilon}\right)^{2} \bar{b}_{H}\left(v-v_{1}\right) M_{1} \mathrm{~d} v_{1} M \mathrm{~d} v\right)^{1 / 2} \\
& \leq C\left\|\phi_{\epsilon}\right\|_{L^{2}\left(\left(1+a_{H}(v)\right) M \mathrm{~d} v\right)}^{2}\left\|\mathcal{P}^{\perp} \phi_{\epsilon}\right\|_{L^{2}\left(a_{H}(v) M \mathrm{~d} v\right)} .
\end{aligned}
$$

Moreover, for almost every $x$ and $t$, we have

$$
\begin{aligned}
\left\|\phi_{\epsilon}\right\|_{L^{2}\left(\left(1+a_{H}(v)\right) M \mathrm{~d} v\right)} & \leq\left\|\phi_{\epsilon}\right\|_{L^{2}(M \mathrm{~d} v)}+\left\|\mathcal{P} \phi_{\epsilon}\right\|_{L^{2}\left(a_{H}(v) M \mathrm{~d} v\right)}+\left\|\mathcal{P}^{\perp} \phi_{\epsilon}\right\|_{L^{2}\left(a_{H}(v) M \mathrm{~d} v\right)} \\
(9.14) & \leq C\left\|\phi_{\epsilon}\right\|_{L^{2}(M \mathrm{~d} v)}+\left\|\mathcal{P}^{\perp} \phi_{\epsilon}\right\|_{L^{2}\left(a_{H}(v) M \mathrm{~d} v\right)} .
\end{aligned}
$$

Hence, we obtain

$$
\begin{aligned}
\left\|\mathcal{P}^{\perp} \phi_{\epsilon}\right\|_{L^{2}\left(a_{H}(v) M \mathrm{~d} v\right) \leq} & \left.\frac{C}{\epsilon} \|\left\langle\log \left(\frac{G_{1}^{\prime} G^{\prime}}{G_{1} G}\right)\left(G_{1}^{\prime} G^{\prime}-G_{1} G\right)\right\rangle\right\rangle^{1 / 2} \\
& +C \epsilon\left[\left\|\phi_{\epsilon}\right\|_{L^{2}(M \mathrm{~d} v)}^{2}+\left\|\mathcal{P}^{\perp} \phi_{\epsilon}\right\|_{\left.L^{2}\left(a_{H}(v) M \mathrm{~d} v\right)\right]}^{2} .\right.
\end{aligned}
$$


This inequality gives us an estimate for $\left\|\mathcal{P}^{\perp} \phi_{\epsilon}\right\|_{L^{2}\left(a_{H}(v) M \mathrm{~d} v\right)}$ only if we know a priori that $\left\|\mathcal{P}^{\perp} \phi_{\epsilon}\right\|_{L^{2}\left(a_{H}(v) M \mathrm{~d} v\right)}$ is not very big.

Let us introduce the macroscopic cut-off given by

$$
A_{\lambda}=\left\{(x, t) \mid \int\left(G_{\epsilon} \log \left(G_{\epsilon}\right)-G_{\epsilon}+1\right) M \mathrm{~d} v \leq \lambda\right\}
$$

Using the following elementary inequality, $\frac{1}{3} x^{2} \leq(1+x)^{2} \log \left[(1+x)^{2}\right]-$ $(1+x)^{2}+1$, we deduce that for every $(x, t) \in A_{\lambda}$, we have $\int \phi_{\epsilon}^{2}<\frac{C \lambda}{\epsilon^{2}}$.

Moreover, $g_{\epsilon}=2 \phi_{\epsilon}+\epsilon \phi_{\epsilon}^{2}$. Hence

$$
\phi_{\epsilon}^{2}(1+a(v)) \leq \frac{C}{\epsilon}\left(g_{\epsilon}-2 \phi_{\epsilon}\right)(1+a(v)) \leq \frac{2 C}{\epsilon}(1+a(v))\left|g_{\epsilon}\right| .
$$

We recall the definition of $h(z)=(1+z) \log (1+z)-z$. Using Young inequality, we get for all $\alpha>0$

$$
\left|g_{\epsilon}\right| \frac{\left(1+|v|^{2}\right)}{4} \leq \frac{\alpha}{\epsilon^{2}} h\left(\epsilon g_{\epsilon}\right)+\frac{\alpha}{\epsilon^{2}} h^{*}\left(\frac{\epsilon}{\alpha} \frac{\left(1+|v|^{2}\right)}{4}\right) .
$$

By taking $\alpha=\frac{\epsilon}{\sqrt{\lambda}}$ and using the superquadratic homogeneity of $h^{*}$, we deduce that

$$
\left|g_{\epsilon}\right||v|^{2} \leq \frac{1}{\epsilon \sqrt{\lambda}} h\left(\epsilon g_{\epsilon}\right)+\frac{\sqrt{\lambda}}{\epsilon} h^{*}\left(\frac{\left(1+|v|^{2}\right)}{4}\right) .
$$

Integrating in $v$, we deduce that

$$
1_{A_{\lambda}} \int\left|g_{\epsilon}\right|\left(1+|v|^{2}\right) M \mathrm{~d} v \leq C \frac{\sqrt{\lambda}}{\epsilon} .
$$

Hence, for every $(x, t) \in A_{\lambda}$, we have

$$
\left\|\mathcal{P}^{\perp} \phi_{\epsilon}\right\|_{L^{2}\left(a_{H}(v) M \mathrm{~d} v\right)} \leq C\left(\int \phi_{\epsilon}^{2}(1+a(v)) M \mathrm{~d} v\right)^{1 / 2} \leq \frac{C \lambda^{1 / 4}}{\epsilon}
$$

Using this estimate in the right hand side of (9.15) and using that $\lambda$ is small enough, we deduce that

$$
\left\|1_{A_{\lambda}} \mathcal{P}^{\perp} \phi_{\epsilon}\right\|_{L_{l o c}^{1}\left(\mathrm{~d} t ; L^{1}\left(\mathrm{~d} x ; L^{2}\left(a_{H}(v) M \mathrm{~d} v\right)\right)\right)} \leq C \epsilon .
$$

Now, we want to compute $\int_{0}^{T} \int_{\mathbb{T}^{D}} \int 1_{\{|v|>R\}}(1+a(v)) \phi_{\epsilon}^{2} \mathrm{~d} t \mathrm{~d} x M \mathrm{~d} v$.

Let us use that

$$
\begin{aligned}
1_{\{|v|>R\}}(1+a(v)) \phi_{\epsilon}^{2}= & \left(1-1_{A_{\lambda}}\right) 1_{\{|v|>R\}}(1+a(v)) \phi_{\epsilon}^{2} \\
& +1_{A_{\lambda}} 1_{\{|v|>R\}}(1+a(v)) \phi_{\epsilon}^{2} .
\end{aligned}
$$

We start by computing the integral of the first term. Notice first that, by the entropy inequality, we deduce that for all $t, \int\left(1-1_{A_{\lambda}}\right) \mathrm{d} x M \mathrm{~d} v \leq$ $\frac{C \epsilon^{2}}{\lambda}$. Using Young inequality, we get 


$$
\begin{aligned}
\frac{1}{\epsilon} & \left(1-1_{A_{\lambda}}\right) 1_{\{|v|>R\}} \frac{(1+a(v))}{8} g_{\epsilon} \\
& \leq \frac{\alpha}{\epsilon^{3}}\left[h\left(\epsilon g_{\epsilon}\right)+\left(1-1_{A_{\lambda}}\right) 1_{\{|v|>R\}} h^{*}\left(\frac{\epsilon}{\alpha} \frac{(1+a(v))}{8}\right)\right] \\
& \leq \frac{\alpha}{\epsilon^{3}}\left[h\left(\epsilon g_{\epsilon}\right)+\left(1-1_{A_{\lambda}}\right) 1_{\{|v|>R\}} h^{*}\left(\frac{\epsilon}{\alpha} \beta_{R} \frac{\left(1+|v|^{2}\right)}{4}\right)\right] \\
& \leq \frac{\alpha}{\epsilon^{3}} h\left(\epsilon g_{\epsilon}\right)+\frac{\beta_{R}^{2}}{\epsilon \alpha}\left(1-1_{A_{\lambda}}\right) h^{*}\left(\frac{\left(1+|v|^{2}\right)}{4}\right) \\
& \leq \frac{C \beta_{R}}{\sqrt{\lambda}}\left[\frac{1}{\epsilon^{2}} h\left(\epsilon g_{\epsilon}\right)+\frac{\lambda}{\epsilon^{2}}\left(1-1_{A_{\lambda}}\right) h^{*}\left(\frac{\left(1+|v|^{2}\right)}{4}\right)\right]
\end{aligned}
$$

where $\beta_{R}=\sup _{|v| \geq R} \frac{1+|a(v)|}{1+|v|^{2}}$ goes to zero when $R$ goes to infinity and we have taken $\alpha=\frac{\epsilon \beta_{R}}{\sqrt{\lambda}}$ and used the superquadratic homogeneity of $h^{*}$. Upon integrating over $v$ and $x$, we get that for every $t$,

$$
\int_{\mathbb{T}^{D}} \int\left(1-1_{A_{\lambda}}\right) 1_{\{|v|>R\}}(1+a(v)) \phi_{\epsilon}^{2} M \mathrm{~d} v \mathrm{~d} x \leq \frac{C \beta_{R}}{\sqrt{\lambda}} .
$$

On the other hand, we have

$\int 1_{\{|v|>R\}}(1+a(v))\left(\mathcal{P} \phi_{\epsilon}\right)^{2} M \mathrm{~d} v \mathrm{~d} x \leq C\left\|\phi_{\epsilon}\right\|_{L^{2}(M \mathrm{~d} v)}^{2} \int_{\{|v|>R\}}\left(1+|v|^{6}\right) M \mathrm{~d} v$,

and

$$
\begin{aligned}
& \left\|1_{A_{\lambda}}\left(\mathcal{P}^{\perp} \phi_{\epsilon}\right)^{2}\right\|_{L_{x, t}^{1}\left(L^{1}(1+a(v)) M \mathrm{~d} v\right)} \\
& \quad \leq\left\|1_{A_{\lambda}} \mathcal{P}^{\perp} \phi_{\epsilon}\right\|_{L_{x, t}^{\infty}\left(L^{2}(1+a(v)) M \mathrm{~d} v\right)}\left\|1_{A_{\lambda}} \mathcal{P}^{\perp} \phi_{\epsilon}\right\|_{L_{x, t}^{1}\left(L^{2}(1+a(v)) M \mathrm{~d} v\right)} \\
& \quad \leq C \frac{\lambda^{1 / 4}}{\epsilon} \epsilon=C \lambda^{1 / 4} .
\end{aligned}
$$

By combining (9.23), (9.24) and (9.25), we complete the proof of the lemma.

Proof of Lemma 9.1. To prove the first statement, we will use the averaging lemma of Golse and Saint-Raymond [24].

Let $\gamma$ be a $C^{\infty}$ cut-off function that satisfies $\gamma(s)=1$ for $0 \leq s \leq 1$, $0 \leq \gamma \leq 1$ and $\gamma(s)=0$ for $s \geq 2$. Using the entropy inequality, we see that for all $\delta>0$, we have

$$
\left\|\frac{g_{\epsilon}^{2}}{n_{\epsilon}}-\frac{g_{\epsilon}^{2}}{n_{\epsilon}} \gamma\left(\delta \frac{\epsilon^{2} g_{\epsilon}^{2}}{n_{\epsilon}}\right)\right\|_{L^{\infty}\left(\mathrm{d} t ; L^{1}(M \mathrm{~d} v \mathrm{~d} x)\right)} \leq \frac{C}{|\log (\delta)|} .
$$


Indeed,

$$
\frac{g_{\epsilon}^{2}}{n_{\epsilon}}-\frac{g_{\epsilon}^{2}}{n_{\epsilon}} \gamma\left(\delta \frac{\epsilon^{2} g_{\epsilon}^{2}}{n_{\epsilon}}\right) \leq \frac{g_{\epsilon}^{2}}{n_{\epsilon}} 1_{\left\{\delta \epsilon g_{\epsilon} \geq 1\right\}} \leq \frac{1}{\epsilon^{2}} G_{\epsilon} 1_{\left\{G_{\epsilon} \geq \frac{1}{\delta}\right\}}
$$

Hence, we only need to prove that $1_{\{|v|<R\}} \psi_{\epsilon}=1_{\{|v|<R\}} \frac{g_{\epsilon}^{2}}{n_{\epsilon}} \gamma\left(\delta \frac{\epsilon^{2} g_{\epsilon}^{2}}{n_{\epsilon}}\right)$ is equi-integrable.

Using the averaging lemma [24], we see that this is a consequence of the fact that $1_{\{|v|<R\}} \psi_{\epsilon}$ is equi-integrable in $v$ (see the definition below) and satisfies

$$
\epsilon \partial_{t} \psi_{\epsilon}+v \cdot \nabla_{x} \psi_{\epsilon}=\frac{\mathcal{Q}\left(G_{\epsilon}, G_{\epsilon}\right)}{\epsilon^{2} \sqrt{n}_{\epsilon}} \frac{g_{\epsilon}}{\sqrt{n}_{\epsilon}} \gamma_{\epsilon}
$$

where $\gamma_{\epsilon}=\frac{1+n_{\epsilon}}{n_{\epsilon}}\left[\gamma\left(\delta \frac{\epsilon^{2} g_{\epsilon}^{2}}{n_{\epsilon}}\right)+\delta \frac{\epsilon^{2} g_{\epsilon}^{2}}{n_{\epsilon}} \gamma^{\prime}\left(\delta \frac{\epsilon^{2} g_{\epsilon}^{2}}{n_{\epsilon}}\right)\right]$ and the right-hand side is bounded in $L_{l o c}^{1}\left(\mathrm{~d} t ; L^{1}(M \mathrm{~d} v \mathrm{~d} x)\right)$.

In the next two steps we prove that $1_{\{|v|<R\}} \frac{g_{\epsilon}^{2}}{n_{\epsilon}}$ is equi-integrable in $v$ and that the right-hand side of (9.26) is bounded in $L_{l o c}^{1}\left(\mathrm{~d} t ; L^{1}(M \mathrm{~d} v \mathrm{~d} x)\right)$.

Step 1: Let us recall the following definition.

Definition 9.1. Take $\psi_{\epsilon}$ a sequence of $L^{1}(M \mathrm{~d} v \mathrm{~d} x \mathrm{~d} t)$. The sequence is said to be equi-integrable in $v$, if and only if for every $\delta>0$ there exists an $\eta>0$ such that for every $\Omega \subset \mathbb{R}^{D} \times \mathbb{R}^{D} \times \mathbb{R}, \Omega$ has a compact support in $x, t$ and $\int_{\mathbb{R}^{D}} 1_{\Omega} M \mathrm{~d} v \leq \eta$ for every $(x, t) \in \mathbb{R}^{D} \times \mathbb{R}$, we have

$$
\int_{\Omega}\left|\psi_{\epsilon}\right| M \mathrm{~d} v \mathrm{~d} x \mathrm{~d} t \leq C \delta .
$$

In the sequel, we will denote such a domain $\Omega$ by

$$
\Omega=\left\{(v, x, t) \mid(x, t) \in \bar{\Omega}, v \in \Omega_{t, x}\right\},
$$

where $\bar{\Omega}$ is bounded in $\mathbb{R}^{D} \times \mathbb{R}$ and for every $(x, t) \in \bar{\Omega}, \int_{\Omega_{x, t}} M \mathrm{~d} v \leq \eta$.

We would like to prove that $\left\|\mathcal{P}^{\perp} \phi_{\epsilon}\right\|_{L_{l o c}^{1}\left(\mathrm{~d} t ; L^{1}\left(\mathrm{~d} x ; L^{2}\left(a_{H}(v) M \mathrm{~d} v\right)\right)\right)} \leq C \epsilon$. This will follow from the proof of lemma 9.2. Indeed, writing (9.22) without the cutoff in $R$ and choosing $\lambda=1$, we deduce that

$\left\|\phi_{\epsilon}\right\|_{L^{2}\left(\left(1+a_{H}(v)\right) \mathrm{d} t \mathrm{~d} x M \mathrm{~d} v\right)} \leq C$. Hence, integrating (9.15) in $x$ and $t$, we see that $\left\|\mathcal{P}^{\perp} \phi_{\epsilon}\right\|_{L_{l o c}^{1}\left(\mathrm{~d} t ; L^{1}\left(\mathrm{~d} x ; L^{2}\left(a_{r}(v) M \mathrm{~d} v\right)\right)\right)} \leq C \epsilon$.

We want to deduce from this that $1_{\{|v|<R\}} \phi_{\epsilon}^{2}$ is equi-integrable in $v$. Take $A>1$, we write

$$
1_{\{|v|<R\}} \phi_{\epsilon}^{2}=1_{\{|v|<R\}} 1_{G_{\epsilon}>A} \phi_{\epsilon}^{2}+1_{\{|v|<R\}} 1_{G_{\epsilon}<A}\left(\phi_{\epsilon} \mathcal{P} \phi_{\epsilon}+\phi_{\epsilon} \mathcal{P}^{\perp} \phi_{\epsilon}\right) .
$$

The first term on the right-hand side can be estimated using the entropy inequality and is of order $\frac{1}{\log A}$ in $L^{\infty}\left(\mathrm{d} t ; L^{1}(M \mathrm{~d} v \mathrm{~d} x)\right)$. The second term 
is bounded in $L^{\infty}\left(\mathrm{d} t ; L^{1}\left(\mathrm{~d} x ; L^{q}(M \mathrm{~d} v)\right)\right.$ for all $1 \leq q<2$. The third term is bounded by $\sqrt{A}$ in $L^{1}\left(\mathrm{~d} x \mathrm{~d} t ; L^{2}(M \mathrm{~d} v)\right)$. Indeed,

$$
\left\|1_{G_{\epsilon}<A} \phi_{\epsilon}\right\|_{L^{\infty}} \leq \frac{\sqrt{A}}{\epsilon} \quad \text { and } \quad\left\|1_{\{|v|<R\}} \mathcal{P}^{\perp} \phi_{\epsilon}\right\|_{L_{x, t}^{1} L^{2}(M \mathrm{~d} v)} \leq C \epsilon .
$$

To prove that $1_{\{|v|<R\}} \phi_{\epsilon}^{2}$ is equi-integrable in $v$, we take a $\delta>0$, then we take $A$ such that $\frac{1}{\log A}<\delta$ and $\eta$ such that $\eta<C \delta^{3}$ and $C \sqrt{A} \eta^{1 / 2}<$ $\delta$. Then, if $\Omega$ satisfies $\int_{\mathbb{R}^{D}} 1_{\Omega} M \mathrm{~d} v \leq \eta$ for every $(x, t) \in \mathbb{T}^{D} \times(0, T)$, we have

$$
\begin{aligned}
& \int_{\bar{\Omega}} \int_{\Omega_{x, t}} 1_{\{|v|<R\}} 1_{G_{\epsilon}<A}\left|\phi_{\epsilon} \mathcal{P} \phi_{\epsilon}\right| M \mathrm{~d} v \mathrm{~d} x \mathrm{~d} t \\
& \quad \leq C \int_{\bar{\Omega}}\left(\int_{\Omega_{x, t}}\left|\phi_{\epsilon} \mathcal{P} \phi_{\epsilon}\right|^{3 / 2} M \mathrm{~d} v\right)^{2 / 3}\left(\int_{\Omega_{x, t}} M \mathrm{~d} v\right)^{1 / 3} \mathrm{~d} x \mathrm{~d} t \\
& \quad \leq C \eta^{1 / 3} \leq \delta .
\end{aligned}
$$

$$
\begin{aligned}
& \int_{\bar{\Omega}} \int_{\Omega_{x, t}} 1_{\{|v|<R\}} 1_{G_{\epsilon}<A}\left|\phi_{\epsilon} \mathcal{P}^{\perp} \phi_{\epsilon}\right| M \mathrm{~d} v \mathrm{~d} x \mathrm{~d} t \\
& \quad \leq C \int_{\bar{\Omega}}\left(\int_{\Omega_{x, t}} 1_{\{|v|<R\}} 1_{G_{\epsilon}<A}\left|\phi_{\epsilon} \mathcal{P}^{\perp} \phi_{\epsilon}\right|^{2} M \mathrm{~d} v\right)^{1 / 2}\left(\int_{\Omega_{x, t}} M \mathrm{~d} v\right)^{1 / 2} \mathrm{~d} x \mathrm{~d} t \\
& \quad \leq C \sqrt{A} \eta^{1 / 2} \leq \delta .
\end{aligned}
$$

Hence, we deduce that $1_{\{|v|<R\}} \phi_{\epsilon}^{2}$ is equi-integrable in $v$ and that $1_{\{|v|<R\}} \frac{g_{\epsilon}^{2}}{n_{\epsilon}}$ is equi-integrable in $v$ since they have the same growth.

Step 2: Now, we want to prove that the right hand side of (9.26) is bounded in $L_{l o c}^{1}\left(\mathrm{~d} t ; L^{1}(M \mathrm{~d} v \mathrm{~d} x)\right)$. Using that

$G_{\epsilon 1}^{\prime} G_{\epsilon}^{\prime}-G_{\epsilon} G_{\epsilon 1}=\left(\sqrt{G_{\epsilon 1}^{\prime} G_{\epsilon}^{\prime}}-\sqrt{G_{\epsilon} G_{\epsilon 1}}\right)^{2}+2\left(\sqrt{G_{\epsilon 1}^{\prime} G_{\epsilon}^{\prime}}-\sqrt{G_{\epsilon} G_{\epsilon 1}}\right) \sqrt{G_{\epsilon} G_{\epsilon 1}}$, we deduce that

$$
\begin{aligned}
\epsilon \partial_{t} \psi_{\epsilon}+v \cdot \nabla_{x} \psi_{\epsilon} \\
=\frac{1}{\epsilon^{2}} \iint\left(\sqrt{G_{\epsilon 1}^{\prime} G_{\epsilon}^{\prime}}-\sqrt{G_{\epsilon} G_{\epsilon 1}}\right)^{2} b \mathrm{~d} \omega M_{1} \mathrm{~d} v_{1} \frac{g_{\epsilon}}{n_{\epsilon}} \gamma_{\epsilon} \\
(9.29)+\frac{2}{\epsilon^{2}} \iint\left(\sqrt{G_{\epsilon 1}^{\prime} G_{\epsilon}^{\prime}}-\sqrt{G_{\epsilon} G_{\epsilon 1}}\right) b \mathrm{~d} \omega M_{1} \mathrm{~d} v_{1} \frac{\sqrt{G_{\epsilon}}}{\sqrt{n_{\epsilon}}} \frac{g_{\epsilon}}{\sqrt{n_{\epsilon}}} \gamma_{\epsilon} \\
+\frac{2}{\epsilon^{2}} \iint\left(\sqrt{G_{\epsilon 1}^{\prime} G_{\epsilon}^{\prime}}-\sqrt{G_{\epsilon} G_{\epsilon 1}}\right) \frac{\sqrt{G_{\epsilon 1}}-1}{\epsilon} b \mathrm{~d} \omega M_{1} \mathrm{~d} v_{1} \frac{\sqrt{G_{\epsilon}}}{\sqrt{n_{\epsilon}}} \frac{\epsilon g_{\epsilon}}{\sqrt{n_{\epsilon}}} \gamma_{\epsilon}
\end{aligned}
$$

Then, we use that $\iint\left(\sqrt{G_{\epsilon 1}^{\prime} G_{\epsilon}^{\prime}}-\sqrt{G_{\epsilon} G_{\epsilon 1}}\right)^{2} b \mathrm{~d} \omega M_{1} \mathrm{~d} v_{1} \in \epsilon^{4} L_{l o c}^{1}\left(\mathrm{~d} t ; L^{1}(M \mathrm{~d} v \mathrm{~d} x)\right)$ and $\frac{\epsilon g_{\epsilon}}{n_{\epsilon}} \in L^{\infty}(M \mathrm{~d} v \mathrm{~d} x \mathrm{~d} t)$ to bound the first term on the right-hand side. 
For the second term, we also use that $\frac{g_{\epsilon}}{\sqrt{n_{\epsilon}}} \in L^{2}(M \mathrm{~d} v \mathrm{~d} x \mathrm{~d} t)$. For the third term, we use that $\frac{\epsilon g_{\epsilon}}{\sqrt{n_{\epsilon}}} \gamma_{\epsilon} \in L^{\infty}(M \mathrm{~d} v \mathrm{~d} x \mathrm{~d} t)$. 


\section{Removal of the Conservation Defects}

The conservation defects have the form

$$
\frac{1}{\epsilon}\left\langle\left\langle\zeta \Gamma_{\epsilon}^{\prime}\left(G_{\epsilon}\right) q_{\epsilon}\right\rangle=\frac{1}{\epsilon}\left\langle\left\langle\zeta\left(\frac{2}{N_{\epsilon}^{2}}-\frac{1}{N_{\epsilon}}\right) q_{\epsilon}\right\rangle,\right.\right.
$$

where $\zeta \in \operatorname{span}\left\{1, v_{1}, \ldots, v_{D},|v|^{2}\right\}$ and $N_{\epsilon}=1+\epsilon^{2} g_{\epsilon}^{2}$. In order to establish momentum and energy conservation laws from the scaled Boltzmann equation we must show that these defects vanish as $\epsilon \rightarrow 0$. This is done with the following proposition.

Proposition 10.1. (Conservation Defect Theorem.) Let $b$ be a collision kernel that satisfies the assumptions of Section 3. Let $G_{\epsilon} \geq 0$ be a family of functions in $C\left([0, \infty) ; w-L^{1}(M \mathrm{~d} v \mathrm{~d} x)\right)$ that satisfies the entropy bound (8.2). Let $g_{\epsilon}$ and $q_{\epsilon}$ be given by (6.1) and (8.3). Let $N_{\epsilon}=1+\epsilon^{2} g_{\epsilon}^{2}$. Assume that the family $g_{\epsilon}$ satisfies

$$
\frac{g_{\epsilon}^{2}}{\sqrt{N_{\epsilon}}} \quad \text { is relatively compact in } w-L_{l o c}^{1}\left(\mathrm{~d} t ; w-L^{1}(a M \mathrm{~d} v \mathrm{~d} x)\right)
$$

Then for $n=1$ and $n=2$ and for every $\zeta \in \operatorname{span}\left\{1, v_{1}, \ldots, v_{D},|v|^{2}\right\}$ one has

$$
\frac{1}{\epsilon}\left\langle\left\langle\zeta \frac{q_{\epsilon}}{N_{\epsilon}^{n}}\right\rangle\right\rangle \rightarrow 0 \quad \text { in } w-L_{l o c}^{1}\left(\mathrm{~d} t ; w-L^{1}(\mathrm{~d} x)\right) \text { as } \epsilon \rightarrow 0 .
$$

10.1. Proof of the Conservation Defect Theorem. The case $n=$ 1 is treated first. The proof simply exploits the collisional symmetries (2.14) and the fact $\zeta$ is a collision invariant to decompose the defect into three parts, each of which is dominated by a function that is then shown to vanish as $\epsilon \rightarrow 0$. The case $n=2$ proceeds similarly, with each part being dominated by the same function that dominates the corresponding part for the $n=1$ case. The estimates on these dominating functions are obtained from the entropy inequality (8.2) through the bound on the dissipation rate and from the compactness hypothesis (10.1).

For the case $n=1$, begin with the elementary decomposition

$$
\left\langle\left\langle\zeta \frac{q_{\epsilon}}{N_{\epsilon}}\right\rangle\right\rangle=\left\langle\left\langle\zeta \frac{\epsilon^{2} g_{\epsilon 1}^{2} q_{\epsilon}}{N_{\epsilon 1} N_{\epsilon}}\right\rangle\right\rangle+\left\langle\left\langle\zeta \frac{q_{\epsilon}}{N_{\epsilon 1} N_{\epsilon}}\right\rangle\right.
$$


Because $\zeta$ is a collision invariant, the collisional symmetries (2.14) can be used to rewrite the second term on the right-hand side of (10.3) as

$$
\begin{aligned}
\left\langle\left\langle\zeta \frac{q_{\epsilon}}{N_{\epsilon 1} N_{\epsilon}}\right\rangle\right. & \left.=\frac{1}{2} \|\left(\zeta+\zeta_{1}\right) \frac{q_{\epsilon}}{N_{\epsilon 1} N_{\epsilon}}\right\rangle \\
& =\frac{1}{4}\left\langle\left(\zeta+\zeta_{1}\right) \frac{N_{\epsilon 1}^{\prime} N_{\epsilon}^{\prime}-N_{\epsilon 1} N_{\epsilon}}{N_{\epsilon 1}^{\prime} N_{\epsilon}^{\prime} N_{\epsilon 1} N_{\epsilon}} q_{\epsilon}\right\rangle .
\end{aligned}
$$

We now observe that

$$
\begin{aligned}
N_{\epsilon 1}^{\prime} N_{\epsilon}^{\prime}-N_{\epsilon 1} N_{\epsilon}= & \epsilon^{2}\left(g_{\epsilon 1}^{\prime 2}+g_{\epsilon}^{\prime 2}-g_{\epsilon 1}^{2}-g_{\epsilon}^{2}\right)+\epsilon^{4}\left(g_{\epsilon 1}^{\prime 2} g_{\epsilon}^{\prime 2}-g_{\epsilon 1}^{2} g_{\epsilon}^{2}\right) \\
= & \epsilon^{2}\left(\left(g_{\epsilon 1}^{\prime}+g_{\epsilon}^{\prime}\right)^{2}-\left(g_{\epsilon 1}+g_{\epsilon}\right)^{2}\right) \\
& -2 \epsilon^{2}\left(g_{\epsilon 1}^{\prime} g_{\epsilon}^{\prime}-g_{\epsilon 1} g_{\epsilon}\right)+\epsilon^{4}\left(g_{\epsilon 1}^{\prime 2} g_{\epsilon}^{\prime 2}-g_{\epsilon 1}^{2} g_{\epsilon}^{2}\right) \\
= & \epsilon^{3} q_{\epsilon}\left(g_{\epsilon 1}^{\prime}+g_{\epsilon}^{\prime}+g_{\epsilon 1}+g_{\epsilon}\right) \\
& -\epsilon^{2}\left(g_{\epsilon 1}^{\prime} g_{\epsilon}^{\prime}-g_{\epsilon 1} g_{\epsilon}\right) J_{\epsilon}
\end{aligned}
$$

where $J_{\epsilon}$ is given by

$$
J_{\epsilon}=2+\epsilon\left(g_{\epsilon 1}^{\prime}+g_{\epsilon}^{\prime}+g_{\epsilon 1}+g_{\epsilon}\right)-\epsilon^{2}\left(g_{\epsilon 1}^{\prime} g_{\epsilon}^{\prime}+g_{\epsilon 1} g_{\epsilon}\right) .
$$

Upon placing (10.5) into (10.4), using collisional symmetries and the fact $\zeta$ is a collision invariant, and placing the result into (10.3), we obtain the decomposition

$$
\begin{aligned}
\frac{1}{\epsilon}\left\langle\left\langle\zeta \frac{q_{\epsilon}}{N_{\epsilon}}\right\rangle\right\rangle= & \left\langle\left\langle\zeta \frac{\epsilon g_{\epsilon 1}^{2} q_{\epsilon}}{N_{\epsilon 1} N_{\epsilon}}\right\rangle+\left\langle\left\langle\zeta \frac{\epsilon^{2}\left(g_{\epsilon 1}+g_{\epsilon}\right) q_{\epsilon}^{2}}{N_{\epsilon 1}^{\prime} N_{\epsilon}^{\prime} N_{\epsilon 1} N_{\epsilon}}\right\rangle\right\rangle\right. \\
& -\left\langle\left\langle\zeta^{\prime} \frac{\epsilon g_{\epsilon 1}^{\prime} g_{\epsilon}^{\prime} q_{\epsilon}}{N_{\epsilon 1}^{\prime} N_{\epsilon}^{\prime} N_{\epsilon 1} N_{\epsilon}} J_{\epsilon}\right\rangle .\right.
\end{aligned}
$$

This decomposition is derived in the same spirit as was decomposition (9.12) in [19]. The difference in the two arises because the role played by $N_{\epsilon}=1+\epsilon^{2} g_{\epsilon}^{2}$ here was played by $n_{\epsilon}=1+\frac{1}{3} \epsilon g_{\epsilon}$ there.

We now dominate the integrands of the three terms on the righthand side of (10.7). Because for every $\zeta \in \operatorname{span}\left\{1, v_{1}, \ldots, v_{D},|v|^{2}\right\}$ there exists a constant $C<\infty$ such that $|\zeta| \leq C \sigma$ where $\sigma(v) \equiv 1+|v|^{2}$, the integrand of the first term is dominated by

$$
\sigma \frac{\epsilon g_{\epsilon 1}^{2}\left|q_{\epsilon}\right|}{N_{\epsilon 1} N_{\epsilon}}
$$

Because

$$
\frac{\epsilon\left|g_{\epsilon 1}+g_{\epsilon}\right|}{\sqrt{N_{\epsilon 1}^{\prime} N_{\epsilon}^{\prime} N_{\epsilon 1} N_{\epsilon}}} \leq 2
$$


the integrand of the second term is dominated by

$$
\sigma \frac{\epsilon q_{\epsilon}^{2}}{\sqrt{N_{\epsilon 1}^{\prime} N_{\epsilon}^{\prime} N_{\epsilon 1} N_{\epsilon}}}
$$

Finally, because

$$
\frac{\left|J_{\epsilon}\right|}{\sqrt{N_{\epsilon 1}^{\prime} N_{\epsilon}^{\prime} N_{\epsilon 1} N_{\epsilon}}} \leq 8
$$

the integrand of the third term is dominated by

$$
\sigma^{\prime} \frac{\epsilon\left|g_{\epsilon 1}^{\prime} g_{\epsilon}^{\prime}\right|\left|q_{\epsilon}\right|}{\sqrt{N_{\epsilon 1}^{\prime} N_{\epsilon}^{\prime} N_{\epsilon 1} N_{\epsilon}}} .
$$

Hence, the result (10.2) for the case $n=1$ will follow once we establish that the terms (10.8), (10.9), and (10.10) vanish as $\epsilon \rightarrow 0$.

The term (10.9) can be treated easily. By the elementary inequality

$$
n_{\epsilon 1}^{\prime} n_{\epsilon}^{\prime} n_{\epsilon 1} n_{\epsilon} \leq 2 \sqrt{N_{\epsilon 1}^{\prime} N_{\epsilon}^{\prime} N_{\epsilon 1} N_{\epsilon}}
$$

it follows directly from estimate (8.12) that

$$
\sigma \frac{\epsilon q_{\epsilon}^{2}}{\sqrt{N_{\epsilon 1}^{\prime} N_{\epsilon}^{\prime} N_{\epsilon 1} N_{\epsilon}}}=O(\epsilon|\log (\epsilon)|) \quad \text { in } L_{l o c}^{1}\left(\mathrm{~d} t ; L^{1}(\mathrm{~d} \mu \mathrm{d} x)\right) \text { as } \epsilon \rightarrow 0 \text {. }
$$

The terms (10.8) and (10.10) require much more work. Lemmas 10.2 and 10.3 respectively will yield the limits

$$
\begin{array}{r}
\sigma \frac{\epsilon g_{\epsilon 1}^{2} q_{\epsilon}}{N_{\epsilon 1} N_{\epsilon}} \rightarrow 0 \quad \text { in } L_{l o c}^{1}\left(\mathrm{~d} t ; L^{1}(\mathrm{~d} \mu \mathrm{d} x)\right) \text { as } \epsilon \rightarrow 0, \\
\sigma^{\prime} \frac{\epsilon g_{\epsilon 1}^{\prime} g_{\epsilon}^{\prime} q_{\epsilon}}{\sqrt{N_{\epsilon 1}^{\prime} N_{\epsilon}^{\prime} N_{\epsilon 1} N_{\epsilon}}} \rightarrow 0 \quad \text { in } L_{l o c}^{1}\left(\mathrm{~d} t ; L^{1}(\mathrm{~d} \mu \mathrm{d} x)\right) \text { as } \epsilon \rightarrow 0,
\end{array}
$$

These lemmas are stated and proved in the next subsection, thereby establishing the result (10.2) for the case $n=1$.

The case $n=2$ follows similarly. Begin with the elementary decomposition

$$
\left\langle\left\langle\zeta \frac{q_{\epsilon}}{N_{\epsilon}^{2}}\right\rangle=\left\langle\left\langle\zeta \frac{\epsilon^{2} g_{\epsilon 1}^{2} q_{\epsilon}}{N_{\epsilon 1} N_{\epsilon}}\left(1+\frac{1}{N_{\epsilon 1}}\right)\right\rangle+\left\langle\left\langle\zeta \frac{q_{\epsilon}}{N_{\epsilon 1}^{2} N_{\epsilon}^{2}}\right\rangle .\right.\right.\right.
$$

Because $\zeta$ is a collision invariant, the collisional symmetries (2.14) can be used to rewrite the second term on the right-hand side of (10.14) as (10.15)

$$
\begin{aligned}
\left\langle\left\langle\zeta \frac{q_{\epsilon}}{N_{\epsilon 1}^{2} N_{\epsilon}^{2}}\right\rangle\right. & \left.=\frac{1}{2}\left\langle\left(\zeta+\zeta_{1}\right) \frac{q_{\epsilon}}{N_{\epsilon 1}^{2} N_{\epsilon}^{2}}\right\rangle\right) \\
& =\frac{1}{4}\left\langle\left(\zeta+\zeta_{1}\right) \frac{N_{\epsilon 1}^{\prime} N_{\epsilon}^{\prime}-N_{\epsilon 1} N_{\epsilon}}{N_{\epsilon 1}^{\prime} N_{\epsilon}^{\prime} N_{\epsilon 1} N_{\epsilon}} \frac{N_{\epsilon 1}^{\prime} N_{\epsilon}^{\prime}+N_{\epsilon 1} N_{\epsilon}}{N_{\epsilon 1}^{\prime} N_{\epsilon}^{\prime} N_{\epsilon 1} N_{\epsilon}} q_{\epsilon}\right\rangle .
\end{aligned}
$$


Upon placing (10.5) into (10.15), using collisional symmetries and the fact $\zeta$ is a collision invariant, and placing the result into (10.14), we obtain the decomposition

$$
\begin{aligned}
\frac{1}{\epsilon}\left\langle\left\langle\zeta \frac{q_{\epsilon}}{N_{\epsilon}^{2}}\right\rangle=\right. & \left\langle\left\langle\zeta \frac{\epsilon g_{\epsilon 1}^{2} q_{\epsilon}}{N_{\epsilon 1} N_{\epsilon}}\left(1+\frac{1}{N_{\epsilon 1}}\right)\right\rangle\right. \\
& +\left\langle\left\langle\zeta \frac{\epsilon^{2}\left(g_{\epsilon 1}+g_{\epsilon}\right) q_{\epsilon}^{2}}{N_{\epsilon 1}^{\prime} N_{\epsilon}^{\prime} N_{\epsilon 1} N_{\epsilon}}\left(\frac{1}{N_{\epsilon 1}^{\prime} N_{\epsilon}^{\prime}}+\frac{1}{N_{\epsilon 1} N_{\epsilon}}\right)\right\rangle\right. \\
& -\left\langle\left\langle\zeta^{\prime} \frac{\epsilon g_{\epsilon 1}^{\prime} g_{\epsilon}^{\prime} q_{\epsilon}}{N_{\epsilon 1}^{\prime} N_{\epsilon}^{\prime} N_{\epsilon 1} N_{\epsilon}} J_{\epsilon}\left(\frac{1}{N_{\epsilon 1}^{\prime} N_{\epsilon}^{\prime}}+\frac{1}{N_{\epsilon 1} N_{\epsilon}}\right)\right\rangle,\right.
\end{aligned}
$$

where $J_{\epsilon}$ is given by (10.6). Because the factors in parentheses above are each bounded by 2 , by arguing as was done for the case $n=1$, the result for the case $n=2$ will also follow upon establishing (10.12) and (10.13). The proof of Proposition 10.1 will therefore be complete upon proving Lemmas 10.2 and 10.3.

10.2. Defect Limit Lemmas. The proofs of Lemmas 10.2 and 10.3 use the compactness hypothesis (10.1) of Proposition 10.1 through the following lemma.

Lemma 10.1. Let $b, g_{\epsilon}$, and $N_{\epsilon}$ be as in Proposition 10.1. Let $s^{*} \in$ $[1, \infty)$ be given by $\frac{1}{s}+\frac{1}{s^{*}}=1$ where $s \in(1, \infty]$ is from the assumed bound (3.15) on $b$. Then for every $w \in L^{s^{*}}(a M \mathrm{~d} v)$ one has that

$$
\frac{w\left(v_{1}\right) g_{\epsilon}^{2}}{\sqrt{N_{\epsilon}}} \text { is relatively compact in } w-L_{l o c}^{1}\left(\mathrm{~d} t ; w-L^{1}(\mathrm{~d} \mu \mathrm{d} x)\right) \text {. }
$$

Proof. By hypothesis (10.1) we can pass to a subsequence such that

$$
\frac{g_{\epsilon}^{2}}{\sqrt{N_{\epsilon}}} \text { is convergent in } w-L_{l o c}^{1}\left(\mathrm{~d} t ; w-L^{1}(a M \mathrm{~d} v \mathrm{~d} x)\right) \text {. }
$$

It is then straightforward to show that the corresponding subsequence

$$
\frac{w\left(v_{1}\right) g_{\epsilon}^{2}}{\sqrt{N_{\epsilon}}} \text { is convergent in } w-L_{l o c}^{1}\left(\mathrm{~d} t ; w-L^{1}(\mathrm{~d} \mu \mathrm{d} x)\right) \text {. }
$$

Indeed, one simply uses the fact that for every $Y \in L_{l o c}^{\infty}\left(\mathrm{d} t ; L^{\infty}(\mathrm{d} \mu \mathrm{d} x)\right)$

$$
\iiint_{\mathbb{S}^{D-1} \times \mathbb{R}^{D \times \mathbb{R}^{D}}} Y \frac{w_{1} g_{\epsilon}^{2}}{\sqrt{N_{\epsilon}}} \mathrm{d} \mu=\int_{\mathbb{R}^{D}} y \frac{g_{\epsilon}^{2}}{\sqrt{N_{\epsilon}}} a M \mathrm{~d} v,
$$

where $y \in L_{l o c}^{\infty}\left(\mathrm{d} t ; L^{\infty}(a M \mathrm{~d} v \mathrm{~d} x)\right)$ is given almost everywhere by

$$
y(v, x, t)=\frac{1}{a(v)} \iint_{\mathbb{S}^{D-1} \times \mathbb{R}^{D}} Y\left(\omega, v_{1}, v, x, t\right) w\left(v_{1}\right) b\left(\omega, v_{1}-v\right) \mathrm{d} \omega M_{1} \mathrm{~d} v_{1} .
$$


The $L^{\infty}$ bound on $y$ follows because for almost every $(v, x, t)$ one sees from the Hölder inequality and from the bound (3.15) on $b$ that

$$
\begin{aligned}
|y(v, x, t)| & \leq\|Y\|_{\infty} \int_{\mathbb{R}^{D}} w\left(v_{1}\right) \frac{\bar{b}\left(v_{1}-v\right)}{a\left(v_{1}\right) a(v)} a\left(v_{1}\right) M_{1} \mathrm{~d} v_{1} \\
& \leq\|Y\|_{\infty}\left(\int_{\mathbb{R}^{D}}\left|w\left(v_{1}\right)\right|^{s^{*}} a\left(v_{1}\right) M_{1} \mathrm{~d} v_{1}\right)^{\frac{1}{s^{*}}} \\
& \left(\int_{\mathbb{R}^{D}}\left|\frac{\bar{b}\left(v_{1}-v\right)}{a\left(v_{1}\right) a(v)}\right|^{s} a\left(v_{1}\right) M_{1} \mathrm{~d} v_{1}\right)^{\frac{1}{s}} \\
& \leq\|Y\|_{\infty}\|w\|_{L^{s^{*}}(a M \mathrm{~d} v)} C_{b} .
\end{aligned}
$$

The compactness result (10.17) then follows.

The proofs of Lemmas 10.2 and 10.3 also crucially use the fact that the entropy inequality (8.2) implies that the dissipation rate $R$ satisfies the bound

$$
\frac{1}{\epsilon^{4}} \int_{0}^{\infty} R\left(G_{\epsilon}\right) \mathrm{d} t \leq C^{i n}
$$

More specifically, following [3], these proofs use the definition of $R$ (2.20) and of $q_{\epsilon}$ (8.3) to re-express this bound as

$$
\frac{1}{\epsilon^{4}} \int_{0}^{\infty} \int\left\langle\left\langle\frac{1}{4} r\left(\frac{\epsilon^{2} q_{\epsilon}}{G_{\epsilon 1} G_{\epsilon}}\right) G_{\epsilon 1} G_{\epsilon}\right\rangle \mathrm{d} x \mathrm{~d} t \leq C^{i n},\right.
$$

where the function $r$ is defined over $z>-1$ by

$$
r(z)=z \log (1+z)
$$

The function $r$ is strictly convex over $z>-1$.

The proofs of Lemmas 10.2 and 10.3 are each based on a delicate use of the classical Young inequality satisfied by $r$ and its Legendre dual, $r^{*}$ — namely, the inequality

$$
p z \leq r^{*}(p)+r(z), \quad \text { for every } p \in \mathbb{R} \text { and } z>-1 .
$$

Upon choosing

$$
p=\frac{\epsilon^{2} y}{\alpha}, \quad \text { and } \quad z=\frac{\epsilon^{2}\left|q_{\epsilon}\right|}{G_{\epsilon 1} G_{\epsilon}},
$$

and noticing that $r(|z|) \leq r(z)$ for every $z>-1$, for every positive $\alpha$ and $y$ one obtains

$$
y\left|q_{\epsilon}\right| \leq \frac{\alpha}{\epsilon^{4}} r^{*}\left(\frac{\epsilon^{2} y}{\alpha}\right) G_{\epsilon 1} G_{\epsilon}+\frac{\alpha}{\epsilon^{4}} r\left(\frac{\epsilon^{2} q_{\epsilon}}{G_{\epsilon 1} G_{\epsilon}}\right) G_{\epsilon 1} G_{\epsilon} .
$$

This inequality will be the starting point for the proofs of Lemmas 10.2 and 10.3. 
These proofs also use the facts, recalled from [3], that $r^{*}$ is superquadratic in the sense

$$
r^{*}(\lambda p) \leq \lambda^{2} r^{*}(p), \quad \text { for every } p>0 \text { and } \lambda \in[0,1],
$$

and that $r^{*}$ has the exponential asymptotics $r^{*}(p) \sim \exp (p)$ as $p \rightarrow \infty$.

Lemma 10.2. Let $b, g_{\epsilon}, q_{\epsilon}$, and $N_{\epsilon}$ be as in Proposition 10.1. Then

$$
\sigma \frac{\epsilon g_{\epsilon 1}^{2} q_{\epsilon}}{N_{\epsilon 1} N_{\epsilon}} \longrightarrow 0 \quad \text { in } L_{l o c}^{1}\left(\mathrm{~d} t ; L^{1}(\mathrm{~d} \mu \mathrm{d} x)\right) \text { as } \epsilon \rightarrow 0 .
$$

Proof. For the proof of this lemma we use inequality (10.20) with

$$
y=\frac{\sigma}{4 s^{*}} \frac{\epsilon g_{\epsilon 1}^{2}}{N_{\epsilon 1} N_{\epsilon}} .
$$

where $s^{*} \in[1, \infty)$ is as in Lemma 10.1. We then apply the superquadratic property (10.21) with

$$
\lambda=\frac{\epsilon^{3} g_{\epsilon 1}^{2}}{\alpha N_{\epsilon 1} N_{\epsilon}} \quad \text { and } \quad p=\frac{\sigma}{4 s^{*}},
$$

where we note that $\lambda \leq 1$ whenever $\epsilon \leq \alpha$. This leads to

$$
\begin{aligned}
\frac{\sigma}{4 s^{*}} \frac{\epsilon g_{\epsilon 1}^{2}\left|q_{\epsilon}\right|}{N_{\epsilon 1} N_{\epsilon}} \leq & \frac{1}{\alpha} \frac{\epsilon^{2} g_{\epsilon 1}^{4}}{N_{\epsilon 1}^{2} N_{\epsilon}^{2}} r^{*}\left(\frac{\sigma}{4 s^{*}}\right) G_{\epsilon 1} G_{\epsilon} \\
& +\frac{\alpha}{\epsilon^{4}} r\left(\frac{\epsilon^{2} q_{\epsilon}}{G_{\epsilon 1} G_{\epsilon}}\right) G_{\epsilon 1} G_{\epsilon} .
\end{aligned}
$$

Because $G_{\epsilon 1} G_{\epsilon} \leq 2 \sqrt{N_{\epsilon 1} N_{\epsilon}}$ while $1 \leq N_{\epsilon}$, the first term on the righthand side above is bounded by

$$
\frac{2 \epsilon^{2} g_{\epsilon 1}^{2}}{\alpha N_{\epsilon 1}} \frac{g_{\epsilon 1}^{2}}{\sqrt{N_{\epsilon 1}}} r^{*}\left(\frac{\sigma}{4 s^{*}}\right) .
$$

The first factor above is bounded by $2 / \alpha$ and tends to zero almost everywhere as $\epsilon \rightarrow 0$. Because $r^{*}(p) \sim \exp (p)$ as $p \rightarrow \infty$ one sees that $r^{*}\left(\sigma / 4 s^{*}\right) \in L^{s^{*}}(a M \mathrm{~d} v)$. We can then use the compactness result (10.17) of Lemma 10.1 with $w=r^{*}\left(\sigma / 4 s^{*}\right)$ to conclude that the remaining factors satisfy

$$
\frac{g_{\epsilon 1}^{2}}{\sqrt{N_{\epsilon 1}}} r^{*}\left(\frac{\sigma}{4 s^{*}}\right) \text { is relatively compact in } w-L_{l o c}^{1}\left(\mathrm{~d} t ; w-L^{1}(\mathrm{~d} \mu \mathrm{d} x)\right) \text {. }
$$

The first term on the right-hand side of (10.22) will thereby converge to zero in $L_{l o c}^{1}\left(\mathrm{~d} t ; L^{1}(\mathrm{~d} \mu \mathrm{d} x)\right)$ as $\epsilon \rightarrow 0$ by the Product Limit Theorem of [3]. On the other hand, the dissipation bound (10.18) implies that the integral of the second term on the right-hand side of (10.22) is bounded by $4 \alpha C^{i n}$. The Lemma therefore follows from the arbitrariness of $\alpha$. 
Lemma 10.3. Let $b, g_{\epsilon}, q_{\epsilon}$, and $N_{\epsilon}$ be as in Proposition 10.1. Then

$$
\sigma \frac{\epsilon g_{\epsilon 1} g_{\epsilon} q_{\epsilon}}{\sqrt{N_{\epsilon 1}^{\prime} N_{\epsilon}^{\prime} N_{\epsilon 1} N_{\epsilon}}} \longrightarrow 0 \quad \text { in } L_{l o c}^{1}\left(\mathrm{~d} t ; L^{1}(\mathrm{~d} \mu \mathrm{d} x)\right) \text { as } \epsilon \rightarrow 0 \text {. }
$$

Proof. For the proof of this lemma we use inequality (10.20) with

$$
y=\frac{\sigma^{\prime}}{4 s^{*}} \frac{\epsilon\left|g_{\epsilon 1}^{\prime} g_{\epsilon}^{\prime}\right|}{\sqrt{N_{\epsilon 1}^{\prime} N_{\epsilon}^{\prime} N_{\epsilon 1} N_{\epsilon}}} .
$$

where $s^{*} \in[1, \infty)$ is as in Lemma 10.1. We then apply the superquadratic property (10.21) with

$$
\lambda=\frac{\epsilon^{3}\left|g_{\epsilon 1}^{\prime} g_{\epsilon}^{\prime}\right|}{\alpha \sqrt{N_{\epsilon 1}^{\prime} N_{\epsilon}^{\prime} N_{\epsilon 1} N_{\epsilon}}}, \quad \text { and } \quad p=\frac{\sigma^{\prime}}{4 s^{*}} .
$$

where we note that $\lambda \leq 1$ whenever $\epsilon \leq \alpha$. This leads to

$$
\begin{aligned}
\frac{\sigma^{\prime}}{4 s^{*}} \frac{\epsilon\left|g_{\epsilon 1}^{\prime} g_{\epsilon}^{\prime}\right|\left|q_{\epsilon}\right|}{\sqrt{N_{\epsilon 1}^{\prime} N_{\epsilon}^{\prime} N_{\epsilon 1} N_{\epsilon}} \leq} & \frac{1}{\alpha} \frac{\epsilon^{2} g_{\epsilon 1}^{\prime 2} g_{\epsilon}^{\prime 2}}{N_{\epsilon 1}^{\prime} N_{\epsilon}^{\prime} N_{\epsilon 1} N_{\epsilon}} r^{*}\left(\frac{\sigma^{\prime}}{4 s^{*}}\right) G_{\epsilon 1} G_{\epsilon} \\
& +\frac{\alpha}{\epsilon^{4}} r\left(\frac{\epsilon^{2} q_{\epsilon}}{G_{\epsilon 1} G_{\epsilon}}\right) G_{\epsilon 1} G_{\epsilon} .
\end{aligned}
$$

Because $G_{\epsilon 1} G_{\epsilon} \leq 2 \sqrt{N_{\epsilon 1} N_{\epsilon}}$ while $1 \leq N_{\epsilon}$, the first term on the righthand side above is bounded by

$$
\frac{2 \epsilon^{2} g_{\epsilon}^{\prime 2}}{\alpha N_{\epsilon}^{\prime}} \frac{g_{\epsilon 1}^{\prime 2}}{N_{\epsilon 1}^{\prime}} r^{*}\left(\frac{\sigma^{\prime}}{4 s^{*}}\right)
$$

The first factor above is bounded by $2 / \alpha$ and tends to zero almost everywhere as $\epsilon \rightarrow 0$. The compactness result (10.17) of Lemma 10.1 with $w=r^{*}\left(\sigma / 4 s^{*}\right)$ implies that the remaining factors satisfy

$$
\frac{g_{\epsilon 1}^{\prime 2}}{\sqrt{N_{\epsilon 1}^{\prime}}} r^{*}\left(\frac{\sigma^{\prime}}{4 s^{*}}\right) \quad \text { is relatively compact in } w-L_{l o c}^{1}\left(\mathrm{~d} t ; w-L^{1}(\mathrm{~d} \mu \mathrm{d} x)\right) \text {. }
$$

The first term on the right-hand side of (10.23) will thereby converge to zero in $L_{l o c}^{1}\left(\mathrm{~d} t ; L^{1}(\mathrm{~d} \mu \mathrm{d} x)\right)$ as $\epsilon \rightarrow 0$ by the Product Limit Theorem of [3]. On the other hand, the dissipation bound (10.18) implies that the integral of the second term on the right-hand side of (10.23) is bounded by $4 \alpha C^{i n}$. The Lemma therefore follows from the arbitrariness of $\alpha$. 


\section{Bilinear Estimates}

Key tools in our work are the following two lemmas dedicated to controlling terms that are quadratic in $\tilde{g}_{\epsilon}$. The first lemma provides a direct $L^{1}$ bound on such terms.

Lemma 11.1. Let the collision kernel $b$ satisfy assumption (3.15) for some $C_{b}<\infty$ and $s \in(1, \infty]$. Let $p=2+\frac{1}{s-1}$, so $p=2$ when $s=\infty$.

Let $\Xi=\Xi\left(\omega, v_{1}, v\right)$ be in $L^{p}(\mathrm{~d} \mu)$ and let $\tilde{g}$ and $\tilde{h}$ be in $L^{2}(a M \mathrm{~d} v)$. Then $\Xi \tilde{g}_{1} \tilde{h}$ is in $L^{1}(\mathrm{~d} \mu)$ and satisfies the $L^{1}$ bound

$$
\left.\left\langle\left|\Xi \tilde{g}_{1} \tilde{h}\right|\right\rangle \leq C_{b}^{\frac{1}{p^{*}}}\left\langle|\Xi|^{p}\right\rangle\right\rangle^{\frac{1}{p}}\left\langle a \tilde{g}^{2}\right\rangle^{\frac{1}{2}}\left\langle a \tilde{h}^{2}\right\rangle^{\frac{1}{2}},
$$

where $\frac{1}{p}+\frac{1}{p^{*}}=1$ and $\tilde{g}_{1}$ denotes $\tilde{g}\left(v_{1}\right)$.

Proof. The Hölder inequality yields

$$
\left\langle\left|\Xi \tilde{g}_{1} \tilde{h}\right|\right\rangle \leq\left\langle\left\langle|\Xi|^{p}\right\rangle\right\rangle^{\frac{1}{p}}\left\langle\left|\tilde{g}_{1} \tilde{h}\right|^{p^{*}}\right\rangle^{\frac{1}{p^{*}}} .
$$

In order to bound the last factor on the left-hand side above, we first observe that

$$
\begin{aligned}
\left\langle\left|\tilde{g}_{1} \tilde{h}\right|^{p^{*}}\right\rangle & =\iint\left|\tilde{g}_{1} \tilde{h}\right|^{p^{*}} \bar{b}\left(v_{1}-v\right) M_{1} \mathrm{~d} v_{1} M \mathrm{~d} v \\
& =\iint K^{-}\left(v_{1}, v\right)\left|\tilde{g}_{1}\right|^{p^{*}}|\tilde{h}|^{p^{*}} a_{1} M_{1} \mathrm{~d} v_{1} a M \mathrm{~d} v \\
& =\left\langle a\left(\mathcal{K}^{-}|\tilde{g}|^{p^{*}}\right)|\tilde{h}|^{p^{*}}\right\rangle,
\end{aligned}
$$

where the integral operator $\mathcal{K}^{-}$and its kernel $K^{-}$are given by (3.17) and (3.18).

Next, let $r=\frac{2}{p^{*}} \in(1,2)$ and $\frac{1}{r}+\frac{1}{r^{*}}=1$. Observe that because $\frac{1}{r}+\frac{1}{s}=1+\frac{1}{r^{*}}$, by $(3.24)$ the operator $\mathcal{K}^{-}: L^{r}(a M \mathrm{~d} v) \rightarrow L^{r^{*}}(a M \mathrm{~d} v)$ is bounded with $\left\|\mathcal{K}^{-}\right\| \leq C_{b}$. Use this fact after another application of the Hölder inequality to find

$$
\begin{aligned}
\left\langle a\left(\mathcal{K}^{-}|\tilde{g}|^{p^{*}}\right)|\tilde{h}|^{p^{*}}\right\rangle & =\left\langle a\left(\mathcal{K}^{-}|\tilde{g}|^{\frac{2}{r}}\right)|\tilde{h}|^{\frac{2}{r}}\right\rangle \\
& \leq\left\|\mathcal{K}^{-}|\tilde{g}|^{\frac{2}{r}}\right\|_{L^{r^{*}}(a M \mathrm{~d} v)}\left\||\tilde{h}|^{\frac{2}{r}}\right\|_{L^{r}(a M \mathrm{~d} v)} \\
& \leq C_{b}\left\langle a \tilde{g}^{2}\right\rangle^{\frac{1}{r}}\left\langle a \tilde{h}^{2}\right\rangle^{\frac{1}{r}}
\end{aligned}
$$

When the above bound is combined with (11.3) we obtain the key bound

$$
\left\langle\left|\tilde{g}_{1} \tilde{h}\right|^{p^{*}}\right\rangle \leq C_{b}\left\langle a \tilde{g}^{2}\right\rangle^{\frac{p^{*}}{2}}\left\langle a \tilde{h}^{2}\right\rangle^{\frac{p^{*}}{2}} .
$$

The $L^{1}$ bound (11.1) then follows when the above inequality is applied to the last factor on the left-hand side of (11.2). 
The next lemma provides $w-L^{1}$ compactness of certain terms quadratic in fluctuations, provided those fluctuations satisfy a weaker compactness hypothesis.

Lemma 11.2. Let the collision kernel b satisfy assumption (3.15) for some $C_{b}<\infty$ and $s \in(1, \infty]$. Let $p=2+\frac{1}{s-1}$, so $p=2$ when $s=\infty$.

Let $\Xi=\Xi\left(\omega, v_{1}, v\right)$ be in $L^{p}(\mathrm{~d} \mu)$ and let $\tilde{g}_{\epsilon}=\tilde{g}_{\epsilon}(v, x, t)$ and $\tilde{h}_{\epsilon}=$ $\tilde{h}_{\epsilon}(v, x, t)$ be families that are bounded in $L_{l o c}^{2}\left(\mathrm{~d} t ; L^{2}(a M \mathrm{~d} v \mathrm{~d} x)\right)$. If the family

$$
\left\langle a \tilde{g}_{\epsilon}^{2}\right\rangle \text { is relatively compact in } w-L_{l o c}^{1}\left(\mathrm{~d} t ; w-L^{1}(\mathrm{~d} x)\right),
$$

then the family

$$
\Xi \tilde{g}_{\epsilon 1} \tilde{h}_{\epsilon} \quad \text { is relatively compact in } w-L_{l o c}^{1}\left(\mathrm{~d} t ; w-L^{1}(\mathrm{~d} \mu \mathrm{d} x)\right) .
$$

Here $\tilde{g}_{\epsilon 1}$ denotes $\tilde{g}_{\epsilon}\left(v_{1}, x, t\right)$.

Proof. To establish the $w-L^{1}$ compactness assertion (11.6) we must show that the family $\Xi \tilde{g}_{\epsilon 1} \tilde{h}_{\epsilon}$ is equi-integrable. Begin with the classical Young's inequality, which for every $\eta>0$ yields

$$
\left|\Xi \tilde{g}_{\epsilon 1} \tilde{h}_{\epsilon}\right| \leq \frac{\eta^{p}}{p}|\Xi|^{p}+\frac{1}{p^{*} \eta^{p^{*}}}\left|\tilde{g}_{\epsilon 1} \tilde{h}_{\epsilon}\right|^{p^{*}}
$$

Now let $\alpha>0$ be arbitrary and set $\eta=\left\langle a \tilde{g}_{\epsilon}^{2}\right\rangle^{\frac{1}{p}} / \alpha$ above to obtain

$$
\left|\Xi \tilde{g}_{\epsilon 1} \tilde{h}_{\epsilon}\right| \leq \frac{1}{p \alpha^{p}}|\Xi|^{p}\left\langle a \tilde{g}_{\epsilon}^{2}\right\rangle+\frac{\alpha^{p^{*}}}{p^{*}} \frac{\left|\tilde{g}_{\epsilon 1} \tilde{h}_{\epsilon}\right|^{p^{*}}}{\left\langle a \tilde{g}_{\epsilon}^{2}\right\rangle^{\frac{p^{*}}{p}}} .
$$

The last term on the right-hand side above is a bounded family in $L_{l o c}^{1}\left(\mathrm{~d} t ; L^{1}(\mathrm{~d} \mu \mathrm{d} x)\right)$ because by the key bound (11.4) of Lemma 11.1 one has

$$
\begin{aligned}
\frac{\left.\|\left|\tilde{g}_{\epsilon 1} \tilde{h}_{\epsilon}\right|^{p^{*}}\right\rangle}{\left\langle a \tilde{g}_{\epsilon}^{2}\right\rangle^{\frac{p^{*}}{p}}} & \leq C_{b}\left\langle a \tilde{g}_{\epsilon}^{2}\right\rangle^{\frac{p^{*}}{2}-\frac{p^{*}}{p}}\left\langle a \tilde{h}_{\epsilon}^{2}\right\rangle^{\frac{p^{*}}{2}} \\
& =C_{b}\left\langle a \tilde{g}_{\epsilon}^{2}\right\rangle^{\frac{1}{r^{*}}}\left\langle a \tilde{h}_{\epsilon}^{2}\right\rangle^{\frac{1}{r}} \\
& \leq C_{b}\left[\frac{1}{r^{*}}\left\langle a \tilde{g}_{\epsilon}^{2}\right\rangle+\frac{1}{r}\left\langle a \tilde{h}_{\epsilon}^{2}\right\rangle\right] .
\end{aligned}
$$

Because $\tilde{g}_{\epsilon}$ and $\tilde{h}_{\epsilon}$ are bounded families in $L_{l o c}^{2}\left(\mathrm{~d} t ; L^{2}(a M \mathrm{~d} v \mathrm{~d} x)\right)$, the last expression above is clearly bounded in $L_{l o c}^{1}\left(\mathrm{~d} t ; L^{1}(\mathrm{~d} x)\right)$. 
Next, we integrate inequality (11.7) over an arbitrary measurable set $\Omega \subset \mathbb{S}^{D-1} \times \mathbb{R}^{D} \times \mathbb{R}^{D} \times \mathbb{T}^{D} \times[0, T]$ and use (11.8) to obtain

$$
\begin{aligned}
\iiint_{\Omega}\left|\Xi \tilde{g}_{\epsilon 1} \tilde{g}_{\epsilon}\right| \mathrm{d} \mu \mathrm{d} x \mathrm{~d} t \leq & \frac{1}{p \alpha^{p}} \iiint_{\Omega}|\Xi|^{p}\left\langle a \tilde{g}_{\epsilon}^{2}\right\rangle \mathrm{d} \mu \mathrm{d} x \mathrm{~d} t \\
& +\frac{\alpha^{p^{*}}}{p^{*}} C_{b} \int_{0}^{T} \int_{\mathbb{T}^{D}}\left[\frac{1}{r^{*}}\left\langle a \tilde{g}_{\epsilon}^{2}\right\rangle+\frac{1}{r}\left\langle a \tilde{h}_{\epsilon}^{2}\right\rangle\right] \mathrm{d} x \mathrm{~d} t .
\end{aligned}
$$

We now use this inequality to argue that the left-hand side above can be made arbitrarily small uniformly in $\epsilon$ by picking the measure of $\Omega$ sufficiently small. To begin, because $\tilde{g}_{\epsilon}$ and $\tilde{h}_{\epsilon}$ are bounded families in $L_{l o c}^{2}\left(\mathrm{~d} t ; L^{2}(a M \mathrm{~d} v \mathrm{~d} x)\right)$, the terms on the second line above can be made arbitrarily small uniformly in $\epsilon$ by a suitable choice of $\alpha$. Next, by hypothesis (11.5), $\left\langle a \tilde{g}_{\epsilon}^{2}\right\rangle$ is equi-integrable with respect to $\mathrm{d} x \mathrm{~d} t$ over $\mathbb{T}^{D} \times[0, T]$ while, because $\Xi \in L^{p}(\mathrm{~d} \mu),|\Xi|^{p}$ is integrable with respect to $\mathrm{d} \mu$ over $\mathbb{S}^{D-1} \times \mathbb{R}^{D} \times \mathbb{R}^{D}$, one thereby sees that $|\Xi|^{p}\left\langle a \tilde{g}_{\epsilon}^{2}\right\rangle$ is equiintegrable with respect to $\mathrm{d} \mu \mathrm{d} x \mathrm{~d} t$ over $\mathbb{S}^{D-1} \times \mathbb{R}^{D} \times \mathbb{R}^{D} \times \mathbb{T}^{D} \times[0, T]$. The first term on the right-hand side above can therefore be made arbitrarily small uniformly in $\epsilon$ by picking the measure of $\Omega$ sufficiently small. We conclude that the family $\Xi \tilde{g}_{\epsilon 1} \tilde{h}_{\epsilon}$ is equi-integrable with respect to $\mathrm{d} \mu \mathrm{d} x \mathrm{~d} t$, whereby the $w-L^{1}$ compactness assertion (11.6) is established. 


\section{Removal of the Flux Remainders}

The flux remainders have the form

$$
\left\langle\hat{\xi} T_{\epsilon}\right\rangle
$$

where $\hat{\xi}$ is an entry of either $\widehat{A}$ or $\widehat{B}$ and where $T_{\epsilon}$ is defined by

$$
T_{\epsilon}=\frac{q_{\epsilon}}{N_{\epsilon 1}^{\prime} N_{\epsilon}^{\prime} N_{\epsilon 1} N_{\epsilon}}-\frac{1}{\epsilon}\left(\tilde{g}_{\epsilon 1}^{\prime}+\tilde{g}_{\epsilon}^{\prime}-\tilde{g}_{\epsilon 1}-\tilde{g}_{\epsilon}\right)-\left(\tilde{g}_{\epsilon 1}^{\prime} \tilde{g}_{\epsilon}^{\prime}-\tilde{g}_{\epsilon 1} \tilde{g}_{\epsilon}\right) .
$$

In order to establish momentum and energy conservation laws from the scaled Boltzmann equation we must show that these remainders vanish as $\epsilon \rightarrow 0$. This is done with the following proposition.

Proposition 12.1. Flux Remainder Theorem. Let $b$ be a collision kernel that satisfies the assumptions of Section 3. Let $s \in(1, \infty]$ be as in the assumed bound (3.15) on $b$. Let $p=2+1 /(s-1)$, so that $p=2$ when $s=\infty$.

Let $G_{\epsilon} \geq 0$ be a family of functions in $C\left([0, \infty) ; w-L^{1}(M \mathrm{~d} v \mathrm{~d} x)\right)$ that satisfies the entropy bound (8.2). Let $g_{\epsilon}$ and $q_{\epsilon}$ be given by (6.1) and (8.3). Let $N_{\epsilon}=1+\epsilon^{2} g_{\epsilon}^{2}, \tilde{g}_{\epsilon}=g_{\epsilon} / N_{\epsilon}$, and $T_{\epsilon}$ be given by (12.1). Assume that the family $g_{\epsilon}$ satisfies

$$
\left\langle a \frac{g_{\epsilon}^{2}}{N_{\epsilon}}\right\rangle \text { is relatively compact in } w-L_{l o c}^{1}\left(\mathrm{~d} t ; w-L^{1}(\mathrm{~d} x)\right) .
$$

Then for every $\Xi \in L^{p}(\mathrm{~d} \mu)$ one has that $T_{\epsilon}$ given by (12.1) satisfies

$$
\Xi T_{\epsilon} \longrightarrow 0 \quad \text { in } L_{l o c}^{1}\left(\mathrm{~d} t ; L^{1}(\mathrm{~d} \mu \mathrm{d} x)\right) \text { as } \epsilon \rightarrow 0 \text {. }
$$

Proof. The key to the argument is to find a decomposition of $T_{\epsilon}$ for which each component can be bounded by one of the sequences

$$
\frac{\left|g_{\epsilon 1} g_{\epsilon}\right|}{\sqrt{N_{\epsilon 1} N_{\epsilon}}}, \quad \frac{\left|g_{\epsilon 1}^{\prime} g_{\epsilon}^{\prime}\right|}{\sqrt{N_{\epsilon 1}^{\prime} N_{\epsilon}^{\prime}}}, \quad \frac{\left|q_{\epsilon}\right|}{\sqrt{N_{\epsilon 1}^{\prime} N_{\epsilon}^{\prime} N_{\epsilon 1} N_{\epsilon}}},
$$

times a bounded sequence that vanishes almost everywhere as $\epsilon \rightarrow 0$. Assertion (12.3) will then follow from the Product Limit Theorem of [3] upon showing that $|\Xi|$ times each of the sequences in (12.4) is relatively compact in $w-L_{l o c}^{1}\left(\mathrm{~d} t ; w-L^{1}(\mathrm{~d} x)\right)$. For the first two sequences in (12.4) this relative compactness follows from assertion (11.6) of Lemma 11.2 and the compactness hypothesis (12.2). For the last sequence in (12.4) this relative compactness follows directly from the fact that it is bounded in $L^{2}(\mathrm{~d} \mu \mathrm{d} x \mathrm{~d} t)$ by $(8.11)$. 
We begin by decomposing $T_{\epsilon}$ given by (12.1) as

$$
\begin{aligned}
T_{\epsilon}= & \frac{1}{\epsilon^{2}} \frac{G_{\epsilon 1}^{\prime} G_{\epsilon}^{\prime}-G_{\epsilon 1} G_{\epsilon}}{N_{\epsilon 1}^{\prime} N_{\epsilon}^{\prime} N_{\epsilon 1} N_{\epsilon}}-\frac{1}{\epsilon}\left(\frac{g_{\epsilon 1}^{\prime}}{N_{\epsilon 1}^{\prime}}+\frac{g_{\epsilon}^{\prime}}{N_{\epsilon}^{\prime}}-\frac{g_{\epsilon 1}}{N_{\epsilon 1}}-\frac{g_{\epsilon}}{N_{\epsilon}}\right) \\
& -\left(\frac{g_{\epsilon 1}^{\prime}}{N_{\epsilon 1}^{\prime}} \frac{g_{\epsilon}^{\prime}}{N_{\epsilon}^{\prime}}-\frac{g_{\epsilon 1}}{N_{\epsilon 1}} \frac{g_{\epsilon}}{N_{\epsilon}}\right) \\
= & T_{1 \epsilon}-T_{1 \epsilon}^{\prime}+T_{2 \epsilon}-T_{2 \epsilon}^{\prime}+T_{3 \epsilon}-T_{3 \epsilon}^{\prime},
\end{aligned}
$$

where $T_{1 \epsilon}, T_{2 \epsilon}$, and $T_{3 \epsilon}$ are defined by

$$
\begin{aligned}
& T_{1 \epsilon}=\frac{1}{\epsilon}\left(\frac{g_{\epsilon 1}}{N_{\epsilon 1}}+\frac{g_{\epsilon}}{N_{\epsilon}}-\frac{g_{\epsilon 1}+g_{\epsilon}}{N_{\epsilon 1} N_{\epsilon}}\right), \\
& T_{2 \epsilon}=\frac{1}{\epsilon}\left(\frac{g_{\epsilon 1}+g_{\epsilon}}{N_{\epsilon 1} N_{\epsilon}}-\frac{g_{\epsilon 1}+g_{\epsilon}}{N_{\epsilon 1}^{\prime} N_{\epsilon}^{\prime} N_{\epsilon 1} N_{\epsilon}}\right), \\
& T_{3 \epsilon}=\left(\frac{g_{\epsilon 1} g_{\epsilon}}{N_{\epsilon 1} N_{\epsilon}}-\frac{g_{\epsilon 1} g_{\epsilon}}{N_{\epsilon 1}^{\prime} N_{\epsilon}^{\prime} N_{\epsilon 1} N_{\epsilon}}\right),
\end{aligned}
$$

and where $T_{1 \epsilon}^{\prime}, T_{2 \epsilon}^{\prime}$, and $T_{3 \epsilon}^{\prime}$ are defined by simply exchanging the roles of the primed and unprimed quantities in the respective definitions of $T_{1 \epsilon}, T_{2 \epsilon}$, and $T_{3 \epsilon}$.

It is easy to obtain the desired bounds for $T_{1 \epsilon}, T_{1 \epsilon}^{\prime}, T_{3 \epsilon}$, and $T_{3 \epsilon}^{\prime}$. For $T_{1 \epsilon}$ we have

$$
\left|T_{1 \epsilon}\right|=\frac{\epsilon\left|g_{\epsilon 1}+g_{\epsilon}\right|\left|g_{\epsilon 1} g_{\epsilon}\right|}{N_{\epsilon 1} N_{\epsilon}}=\frac{\left|g_{\epsilon 1} g_{\epsilon}\right|}{\sqrt{N_{\epsilon 1} N_{\epsilon}}} \frac{\epsilon\left|g_{\epsilon 1}+g_{\epsilon}\right|}{\sqrt{N_{\epsilon 1} N_{\epsilon}}} .
$$

The last factor above is a sequence that is bounded by 2 and that vanishes almost everywhere as $\epsilon \rightarrow 0$. For $T_{3 \epsilon}$ we have

$$
\left|T_{3 \epsilon}\right|=\frac{\left|g_{\epsilon 1} g_{\epsilon}\right|}{N_{\epsilon 1} N_{\epsilon}}\left(1-\frac{1}{N_{\epsilon 1}^{\prime} N_{\epsilon}^{\prime}}\right) .
$$

The last factor above is a sequence that is bounded by 1 and that vanishes almost everywhere as $\epsilon \rightarrow 0$. The bounds for $T_{1 \epsilon}^{\prime}$ and $T_{3 \epsilon}^{\prime}$ are obtained by simply exchanging the roles of the primed and unprimed quantities in the respective bounds of $T_{1 \epsilon}$ and $T_{3 \epsilon}$.

To treat $T_{2 \epsilon}$ and $T_{2 \epsilon}^{\prime}$ we need the further decompositions

$$
\begin{aligned}
& T_{2 \epsilon}=\frac{\epsilon\left(g_{\epsilon 1}+g_{\epsilon}\right)\left(g_{\epsilon 1}^{\prime 2}+g_{\epsilon}^{\prime 2}+\epsilon^{2}\left(g_{\epsilon 1}^{\prime} g_{\epsilon}^{\prime}\right)^{2}\right)}{N_{\epsilon 1}^{\prime} N_{\epsilon}^{\prime} N_{\epsilon 1} N_{\epsilon}}=T_{4 \epsilon}-T_{5 \epsilon}+T_{6 \epsilon}, \\
& T_{2 \epsilon}^{\prime}=\frac{\epsilon\left(g_{\epsilon 1}^{\prime}+g_{\epsilon}^{\prime}\right)\left(g_{\epsilon 1}^{2}+g_{\epsilon}^{2}+\epsilon^{2}\left(g_{\epsilon 1} g_{\epsilon}\right)^{2}\right)}{N_{\epsilon 1}^{\prime} N_{\epsilon}^{\prime} N_{\epsilon 1} N_{\epsilon}}=T_{4 \epsilon}^{\prime}-T_{5 \epsilon}^{\prime}+T_{6 \epsilon}^{\prime},
\end{aligned}
$$


where $T_{4 \epsilon}, T_{5 \epsilon}$, and $T_{6 \epsilon}$ are defined by

$$
\begin{aligned}
& T_{4 \epsilon}=\frac{\epsilon\left(g_{\epsilon 1}+g_{\epsilon}\right)\left(g_{\epsilon 1}^{\prime}+g_{\epsilon}^{\prime}\right)^{2}}{N_{\epsilon 1}^{\prime} N_{\epsilon}^{\prime} N_{\epsilon 1} N_{\epsilon}}, \\
& T_{5 \epsilon}=\frac{\epsilon\left(g_{\epsilon 1}+g_{\epsilon}\right)\left(2 g_{\epsilon 1}^{\prime} g_{\epsilon}^{\prime}\right)}{N_{\epsilon 1}^{\prime} N_{\epsilon}^{\prime} N_{\epsilon 1} N_{\epsilon}}, \\
& T_{6 \epsilon}=\frac{\epsilon^{3}\left(g_{\epsilon 1}+g_{\epsilon}\right)\left(g_{\epsilon 1}^{\prime} g_{\epsilon}^{\prime}\right)^{2}}{N_{\epsilon 1}^{\prime} N_{\epsilon}^{\prime} N_{\epsilon 1} N_{\epsilon}},
\end{aligned}
$$

and where $T_{4 \epsilon}^{\prime}, T_{5 \epsilon}^{\prime}$, and $T_{6 \epsilon}^{\prime}$ are defined by simply exchanging the roles of the primed and unprimed quantities in the respective definitions of $T_{4 \epsilon}, T_{5 \epsilon}$, and $T_{6 \epsilon}$.

It is easy to obtain the desired bounds for $T_{5 \epsilon}, T_{5 \epsilon}^{\prime}, T_{6 \epsilon}$, and $T_{6 \epsilon}^{\prime}$. For $T_{5 \epsilon}$ we have

$$
\left|T_{5 \epsilon}\right|=\frac{\epsilon\left|g_{\epsilon 1}+g_{\epsilon}\right|\left|2 g_{\epsilon 1}^{\prime} g_{\epsilon}^{\prime}\right|}{N_{\epsilon 1}^{\prime} N_{\epsilon}^{\prime} N_{\epsilon 1} N_{\epsilon}}=\frac{\left|g_{\epsilon 1}^{\prime} g_{\epsilon}^{\prime}\right|}{N_{\epsilon 1}^{\prime} N_{\epsilon}^{\prime}} \frac{\epsilon 2\left|g_{\epsilon 1}+g_{\epsilon}\right|}{N_{\epsilon 1} N_{\epsilon}} .
$$

The last factor above is a sequence that is bounded by 2 and that vanishes almost everywhere as $\epsilon \rightarrow 0$. For $T_{6 \epsilon}$ we have

$$
\left|T_{6 \epsilon}\right|=\frac{\left|g_{\epsilon 1}^{\prime} g_{\epsilon}^{\prime}\right|}{\sqrt{N_{\epsilon 1}^{\prime} N_{\epsilon}^{\prime}}}\left(\frac{\left|g_{\epsilon 1}^{\prime} g_{\epsilon}^{\prime}\right|}{\sqrt{N_{\epsilon 1}^{\prime} N_{\epsilon}^{\prime}}} \frac{\epsilon\left|g_{\epsilon 1}+g_{\epsilon}\right|}{N_{\epsilon 1} N_{\epsilon}}\right) .
$$

The factor in parenthesis above is a sequence that is bounded by 1 and that vanishes almost everywhere as $\epsilon \rightarrow 0$. The bounds for $T_{5 \epsilon}^{\prime}$ and $T_{6 \epsilon}^{\prime}$ are obtained by simply exchanging the roles of the primed and unprimed quantities in the respective bounds of $T_{5 \epsilon}$ and $T_{6 \epsilon}$.

The trick now is to not treat $T_{4 \epsilon}$ and $T_{4 \epsilon}^{\prime}$ separately. Rather, we use the decomposition

$$
\begin{aligned}
T_{4 \epsilon}-T_{4 \epsilon}^{\prime} & =\frac{\epsilon\left(g_{\epsilon 1}+g_{\epsilon}\right)\left(g_{\epsilon 1}^{\prime}+g_{\epsilon}^{\prime}\right)\left(g_{\epsilon 1}^{\prime}+g_{\epsilon}^{\prime}-g_{\epsilon 1}-g_{\epsilon}\right)}{N_{\epsilon 1}^{\prime} N_{\epsilon}^{\prime} N_{\epsilon 1} N_{\epsilon}} \\
& =T_{7 \epsilon}+T_{8 \epsilon}-T_{8 \epsilon}^{\prime},
\end{aligned}
$$

where $T_{7 \epsilon}$, and $T_{8 \epsilon}$ are defined by

$$
\begin{aligned}
& T_{7 \epsilon}=\frac{\epsilon^{2}\left(g_{\epsilon 1}+g_{\epsilon}\right)\left(g_{\epsilon 1}^{\prime}+g_{\epsilon}^{\prime}\right) q_{\epsilon}}{N_{\epsilon 1}^{\prime} N_{\epsilon}^{\prime} N_{\epsilon 1} N_{\epsilon}}, \\
& T_{8 \epsilon}=\frac{\epsilon^{2}\left(g_{\epsilon 1}+g_{\epsilon}\right)\left(g_{\epsilon 1}^{\prime}+g_{\epsilon}^{\prime}\right) g_{\epsilon 1} g_{\epsilon}}{N_{\epsilon 1}^{\prime} N_{\epsilon}^{\prime} N_{\epsilon 1} N_{\epsilon}},
\end{aligned}
$$

and where $T_{8 \epsilon}^{\prime}$ is defined by simply exchanging the roles of the primed and unprimed quantities in the definition of $T_{8 \epsilon}$. 
Finally, it is easy to obtain the desired bounds for $T_{7 \epsilon}, T_{8 \epsilon}$, and $T_{8 \epsilon}^{\prime}$. For $T_{7 \epsilon}$ we have

$$
\left|T_{7 \epsilon}\right|=\frac{\left|q_{\epsilon}\right|}{\sqrt{N_{\epsilon 1}^{\prime} N_{\epsilon}^{\prime} N_{\epsilon 1} N_{\epsilon}}} \frac{\epsilon^{2}\left|g_{\epsilon 1}+g_{\epsilon}\right|\left|g_{\epsilon 1}^{\prime}+g_{\epsilon}^{\prime}\right|}{\sqrt{N_{\epsilon 1}^{\prime} N_{\epsilon}^{\prime} N_{\epsilon 1} N_{\epsilon}}} .
$$

The last factor above is a sequence that is bounded by 4 and that vanishes almost everywhere as $\epsilon \rightarrow 0$. For $T_{8 \epsilon}$ we have

$$
\left|T_{8 \epsilon}\right|=\frac{\left|g_{\epsilon 1} g_{\epsilon}\right|}{\sqrt{N_{\epsilon 1} N_{\epsilon}}}\left(\frac{\epsilon\left|g_{\epsilon 1}+g_{\epsilon}\right|}{\sqrt{N_{\epsilon 1} N_{\epsilon}}} \frac{\epsilon\left|g_{\epsilon 1}^{\prime}+g_{\epsilon}^{\prime}\right|}{N_{\epsilon 1}^{\prime} N_{\epsilon}^{\prime}}\right) .
$$

The factor in parenthesis above is a sequence that is bounded by 2 and that vanishes almost everywhere as $\epsilon \rightarrow 0$. The bound for $T_{8 \epsilon}^{\prime}$ is obtained by simply exchanging the roles of the primed and unprimed quantities in the bound of $T_{8 \epsilon}$.

We therefore obtain from (12.5), (12.6), and (12.7) the decomposition

$$
T_{\epsilon}=T_{1 \epsilon}-T_{1 \epsilon}^{\prime}+T_{3 \epsilon}-T_{3 \epsilon}^{\prime}-T_{5 \epsilon}+T_{5 \epsilon}^{\prime}+T_{6 \epsilon}-T_{6 \epsilon}^{\prime}+T_{7 \epsilon}+T_{8 \epsilon}-T_{8 \epsilon}^{\prime},
$$

with the desired bounds on each component. This proves the Proposition. 


\section{QuAdratic Limits}

In order to establish our main result, Theorem 6.1 , we have to prove some compactness of our sequence $\tilde{g}_{\epsilon}$. More specifically, we need to pass to the limit in certain of the quadratic terms containing

$$
\tilde{u}_{\epsilon} \otimes \tilde{u}_{\epsilon}, \quad \tilde{u}_{\epsilon} \tilde{\theta}_{\epsilon}, \quad \tilde{\theta}_{\epsilon}^{2} .
$$

Recall that we have the weak limits

$$
\left.\begin{array}{l}
\tilde{u}_{\epsilon} \rightarrow u \\
\tilde{\theta}_{\epsilon} \rightarrow \theta
\end{array}\right\} \quad \text { in } w-L_{l o c}^{2}\left(\mathrm{~d} t ; w-L^{2}(\mathrm{~d} x)\right) \text { as } \epsilon \rightarrow 0 .
$$

These limits have to be strengthened in order to pass to the limit in any of the quadratic terms (13.1). We follow [36], which adapted to the kinetic setting an idea introduced in [34] to pass to an incompressible Navier-Stokes-Fourier limit from the compressible Navier-StokesFourier system. The main result of this section is the following.

Proposition 13.1. Quadratic Limits Theorem.

$$
\left.\begin{array}{rl}
\lim _{\epsilon \rightarrow 0} \Pi \nabla_{x} \cdot\left(\tilde{u}_{\epsilon} \otimes \tilde{u}_{\epsilon}\right) & =\Pi \nabla_{x} \cdot(u \otimes u) \\
\lim _{\epsilon \rightarrow 0} \nabla_{x} \cdot\left(\tilde{\theta}_{\epsilon} \tilde{u}_{\epsilon}\right) & =\nabla_{x} \cdot(\theta u)
\end{array}\right\} \quad \text { in } w-L_{l o c}^{1}\left(\mathrm{~d} t ; \mathcal{D}^{\prime}\left(\mathbb{T}^{D}\right)\right),
$$

where $\Pi$ is the Leray projection onto divergence-free vector fields in $L^{2}\left(\mathrm{~d} x ; \mathbb{R}^{D}\right)$.

Proof. We employ a mollifier over the periodic space variable. Recall that $\mathbb{T}^{D}=\mathbb{R}^{D} / \mathbb{L}^{D}$, where $\mathbb{L}^{D} \subset \mathbb{R}^{D}$ is some $D$-dimensional lattice. Let $j \in C^{\infty}\left(\mathbb{R}^{D}\right)$ be such that $j \geq 0, \int_{\mathbb{R}^{D}} j(x) \mathrm{d} x=1$, and $j(x)=0$ for $|x|>1$. We then define $j^{\delta} \in C^{\infty}\left(\mathbb{T}^{D}\right)$ by

$$
j^{\delta}(x)=\frac{1}{\delta^{D}} \sum_{l \in \mathbb{L}^{D}} j\left(\frac{x+l}{\delta}\right) .
$$

In this section all convolutions are taken only in the $x$ variable.

Define $\tilde{u}_{\epsilon}^{\delta}=j^{\delta} * \tilde{u}_{\epsilon}$ and $\tilde{\theta}_{\epsilon}^{\delta}=j^{\delta} * \tilde{\theta}_{\epsilon}$. It will follow from Proposition 13.2 that

$$
\left.\begin{array}{l}
\lim _{\delta \rightarrow 0} \tilde{u}_{\epsilon}^{\delta}=\tilde{u}_{\epsilon} \\
\lim _{\delta \rightarrow 0} \tilde{\theta}_{\epsilon}^{\delta}=\tilde{\theta}_{\epsilon}
\end{array}\right\} \quad \text { in } L_{l o c}^{2}\left(\mathrm{~d} t ; L^{2}(\mathrm{~d} x)\right) \text { uniformly in } \epsilon
$$


It will follow from Proposition 13.3 that for every $\delta>0$ (13.5)

$$
\left.\begin{array}{rl}
\lim _{\epsilon \rightarrow 0} \Pi \nabla_{x} \cdot\left(\tilde{u}_{\epsilon}^{\delta} \otimes \tilde{u}_{\epsilon}^{\delta}\right) & =\Pi \nabla_{x} \cdot\left(u^{\delta} \otimes u^{\delta}\right) \\
\lim _{\epsilon \rightarrow 0} \nabla_{x} \cdot\left(\tilde{\theta}_{\epsilon}^{\delta} \tilde{u}_{\epsilon}^{\delta}\right) & =\nabla_{x} \cdot\left(\theta^{\delta} u^{\delta}\right)
\end{array}\right\} \quad \text { in } w-L_{l o c}^{1}\left(\mathrm{~d} t ; \mathcal{D}^{\prime}\left(\mathbb{T}^{D}\right)\right)
$$

where $u^{\delta}=j^{\delta} * u$ and $u^{\delta}=j^{\delta} * u$.

By first using the uniformity of the $L^{2}$ limits in (13.4) to commute limits and then using the limits (13.5), we obtain

$$
\begin{aligned}
\lim _{\epsilon \rightarrow 0} \Pi \nabla_{x} \cdot\left(\tilde{u}_{\epsilon} \otimes \tilde{u}_{\epsilon}\right) & =\lim _{\epsilon \rightarrow 0} \lim _{\delta \rightarrow 0} \Pi \nabla_{x} \cdot\left(\tilde{u}_{\epsilon}^{\delta} \otimes \tilde{u}_{\epsilon}^{\delta}\right) \\
& =\lim _{\delta \rightarrow 0} \lim _{\epsilon \rightarrow 0} \Pi \nabla_{x} \cdot\left(\tilde{u}_{\epsilon}^{\delta} \otimes \tilde{u}_{\epsilon}^{\delta}\right) \\
& =\lim _{\delta \rightarrow 0} \Pi \nabla_{x} \cdot\left(u^{\delta} \otimes u^{\delta}\right) \\
& =\Pi \nabla_{x} \cdot(u \otimes u) \quad \text { in } w-L_{l o c}^{1}\left(\mathrm{~d} t ; \mathcal{D}^{\prime}\left(\mathbb{T}^{D}\right)\right)
\end{aligned}
$$

and

$$
\begin{aligned}
\lim _{\epsilon \rightarrow 0} \nabla_{x} \cdot\left(\tilde{\theta}_{\epsilon} \tilde{u}_{\epsilon}\right) & =\lim _{\epsilon \rightarrow 0} \lim _{\delta \rightarrow 0} \nabla_{x} \cdot\left(\tilde{\theta}_{\epsilon}^{\delta} \tilde{u}_{\epsilon}^{\delta}\right) \\
& =\lim _{\delta \rightarrow 0} \lim _{\epsilon \rightarrow 0} \nabla_{x} \cdot\left(\tilde{\theta}_{\epsilon}^{\delta} \tilde{u}_{\epsilon}^{\delta}\right) \\
& =\lim _{\delta \rightarrow 0} \nabla_{x} \cdot\left(\theta^{\delta} u^{\delta}\right) \\
& =\nabla_{x} \cdot(\theta u) \quad \text { in } w-L_{l o c}^{1}\left(\mathrm{~d} t ; \mathcal{D}^{\prime}\left(\mathbb{T}^{D}\right)\right)
\end{aligned}
$$

This proves assertion (13.3) and thereby establishes the proposition modulo the proofs of Propositions 13.2 and 13.3, which are given in the subsequent subsections.

13.1. Uniformity of Mollification Limits. Now define $\tilde{g}_{\epsilon}^{\delta}=j^{\delta} * \tilde{g}_{\epsilon}$ and $g^{\delta}=j^{\delta} * g$. By basic properties of mollifiers we know that for every $\xi \in L^{2}(M \mathrm{~d} v)$ one has the $L^{2}$ limits

$$
\left.\begin{array}{l}
\lim _{\delta \rightarrow 0}\left\langle\xi \tilde{g}_{\epsilon}^{\delta}\right\rangle=\left\langle\xi \tilde{g}_{\epsilon}\right\rangle \\
\lim _{\delta \rightarrow 0}\left\langle\xi g^{\delta}\right\rangle=\langle\xi g\rangle
\end{array}\right\} \quad \text { in } L_{l o c}^{2}\left(\mathrm{~d} t ; L^{2}(\mathrm{~d} x)\right)
$$

The main result of this subsection is the following assertion that for certain $\xi$ the first mollification limit above is uniform in $\epsilon$.

Proposition 13.2. For every $\zeta \in \operatorname{span}\left\{1, v_{1}, \ldots, v_{D},|v|^{2}\right\}$ one has

$$
\lim _{\delta \rightarrow 0}\left\langle\zeta \tilde{g}_{\epsilon}^{\delta}\right\rangle=\left\langle\zeta \tilde{g}_{\epsilon}\right\rangle \quad \text { in } L_{l o c}^{2}\left(\mathrm{~d} t ; L^{2}(\mathrm{~d} x)\right) \text { uniformly in } \epsilon \text {. }
$$


Proof. Assertion (a) of Lemma 8.2, which is a consequence of velocity averaging, implies that for every $T \in(0, \infty)$ one has

$$
\lim _{y \rightarrow 0} \iint_{\mathbb{T}^{D \times[0, T]}}\left|\left\langle\zeta \tilde{g}_{\epsilon}\right\rangle(x-y, t)-\left\langle\zeta \tilde{g}_{\epsilon}\right\rangle(x, t)\right| \mathrm{d} x \mathrm{~d} t=0 \quad \text { uniformly in } \epsilon .
$$

Because

$$
\begin{aligned}
& \iint_{\mathbb{T}^{D} \times[0, T]}\left|\left\langle\zeta \tilde{g}_{\epsilon}^{\delta}\right\rangle-\left\langle\zeta \tilde{g}_{\epsilon}\right\rangle\right| \mathrm{d} x \mathrm{~d} t \\
& \leq \iiint_{\mathbb{T}^{D \times \mathbb{T}^{D} \times[0, T]}}\left|\left\langle\zeta \tilde{g}_{\epsilon}\right\rangle(x-y, t)-\left\langle\zeta \tilde{g}_{\epsilon}\right\rangle(x, t)\right| j^{\delta}(y) \mathrm{d} y \mathrm{~d} x \mathrm{~d} t,
\end{aligned}
$$

it therefore follows that

$$
\lim _{\delta \rightarrow 0}\left\langle\zeta \tilde{g}_{\epsilon}^{\delta}\right\rangle=\left\langle\zeta \tilde{g}_{\epsilon}\right\rangle \quad \text { in } L_{l o c}^{1}\left(\mathrm{~d} t ; L^{1}(\mathrm{~d} x)\right) \text { uniformly in } \epsilon .
$$

This is the $L^{1}$ analog of assertion (13.7).

In order to replace $L^{1}$ by $L^{2}$ in (13.8) we now use the fact that $\left\langle a \tilde{g}_{\epsilon}^{2}\right\rangle$ is relatively compact in $w-L_{l o c}^{1}\left(\mathrm{~d} t ; w-L^{1}(\mathrm{~d} x)\right)$ to establish the fact that

$$
\left\langle\zeta \tilde{g}_{\epsilon}^{\delta}\right\rangle^{2} \text { is relatively compact in } w-L_{l o c}^{1}\left(\mathrm{~d} t ; w-L^{1}(\mathrm{~d} x)\right) \text {. }
$$

Indeed, for almost every $(x, t)$ one has the pointwise bound

$$
\begin{aligned}
\left\langle\zeta \tilde{g}_{\epsilon}^{\delta}\right\rangle^{2}(x, t)= & \iint_{\mathbb{T}^{D \times \mathbb{T}^{D}}}\left\langle\zeta \tilde{g}_{\epsilon}\right\rangle\left(y_{1}, t\right)\left\langle\zeta \tilde{g}_{\epsilon}\right\rangle\left(y_{2}, t\right) j^{\delta}\left(x-y_{1}\right) j^{\delta}\left(x-y_{2}\right) \mathrm{d} y_{1} \mathrm{~d} y_{2} \\
\leq & \iint_{\mathbb{T}^{D \times T^{D}}} \frac{1}{2}\left\langle\zeta \tilde{g}_{\epsilon}\right\rangle^{2}\left(y_{1}, t\right) j^{\delta}\left(x-y_{1}\right) j^{\delta}\left(x-y_{2}\right) \mathrm{d} y_{1} \mathrm{~d} y_{2} \\
& +\iint_{\mathbb{T}^{D \times \mathbb{T}^{D}}} \frac{1}{2}\left\langle\zeta \tilde{g}_{\epsilon}\right\rangle^{2}\left(y_{2}, t\right) j^{\delta}\left(x-y_{1}\right) j^{\delta}\left(x-y_{2}\right) \mathrm{d} y_{1} \mathrm{~d} y_{2} \\
= & j^{\delta} *\left(\left\langle\zeta \tilde{g}_{\epsilon}\right\rangle^{2}\right)(x, t) \\
\leq & \left\langle\frac{1}{a} \zeta^{2}\right\rangle j^{\delta} *\left\langle a \tilde{g}_{\epsilon}^{2}\right\rangle(x, t) .
\end{aligned}
$$

Because the family $\left\langle a \tilde{g}_{\epsilon}^{2}\right\rangle$ is relatively compact in $w-L_{l o c}^{1}\left(\mathrm{~d} t ; w-L^{1}(\mathrm{~d} x)\right)$, if follows easily that the doubly indexed family $j^{\delta} *\left\langle a \tilde{g}_{\epsilon}^{2}\right\rangle$ is as well, whereby the above inequality implies that (13.9) holds.

Assertion (13.7) follows from (13.8) and (13.9) upon applying the following lemma to the doubly indexed family $\left\langle\zeta \tilde{g}_{\epsilon}^{\delta}\right\rangle-\left\langle\zeta \tilde{g}_{\epsilon}\right\rangle$.

The above proof of Proposition 13.2 required the following lemma. 
Lemma 13.1. Let $\psi_{\epsilon}^{\delta}$ be a family in $L_{l o c}^{2}\left(\mathrm{~d} t ; C^{\infty}\left(\mathbb{T}^{D}\right)\right)$ doubly indexed by $\epsilon>0$ and $\delta>0$ such that

$$
\begin{gathered}
\left(\psi_{\epsilon}^{\delta}\right)^{2} \quad \text { is relatively compact in } w-L_{l o c}^{1}\left(\mathrm{~d} t ; w-L^{1}(\mathrm{~d} x)\right) \\
\lim _{\delta \rightarrow 0} \psi_{\epsilon}^{\delta}=0 \quad \text { in } L_{l o c}^{1}\left(\mathrm{~d} t ; L^{1}(\mathrm{~d} x)\right) \text { uniformly in } \epsilon
\end{gathered}
$$

Then

$$
\lim _{\delta \rightarrow 0} \psi_{\epsilon}^{\delta}=0 \quad \text { in } L_{l o c}^{2}\left(\mathrm{~d} t ; L^{2}(\mathrm{~d} x)\right) \text { uniformly in } \epsilon .
$$

Proof. Let $T \in(0, \infty)$. Because the family $\left(\psi_{\epsilon}^{\delta}\right)^{2}$ is relatively compact in $w-L_{l o c}^{1}\left(\mathrm{~d} t ; w-L^{1}(\mathrm{~d} x)\right)$ one has that

$$
M=\sup \left\{\left(\iint_{\mathbb{T}^{D} \times[0, T]}\left|\psi_{\epsilon}^{\delta}\right|^{2} \mathrm{~d} x \mathrm{~d} t\right)^{\frac{1}{2}}\right\}<\infty .
$$

For every $\lambda>0$ define

$$
\Omega_{\epsilon}^{\delta}(\lambda)=\left\{(x, t) \in \mathbb{T}^{D} \times[0, T]:\left|\psi_{\epsilon}^{\delta}(x, t)\right|>\lambda\right\} .
$$

The Chebychev inequality then yields

$$
\operatorname{meas}\left\{\Omega_{\epsilon}^{\delta}(\lambda)\right\} \leq \frac{M^{2}}{\lambda^{2}} .
$$

Now let $\eta>0$ be arbitrary. Because $\left(\psi_{\epsilon}^{\delta}\right)^{2}$ is relatively compact in $w-L_{l o c}^{1}\left(\mathrm{~d} t ; w-L^{1}(\mathrm{~d} x)\right)$, by the above inequality we may pick $\lambda$ large enough to insure that

$$
\iint_{\Omega_{\epsilon}^{\delta}(\lambda)}\left|\psi_{\epsilon}^{\delta}\right|^{2} \mathrm{~d} x \mathrm{~d} t<\frac{\eta}{2} \quad \text { for every } \epsilon \text { and } \delta .
$$

The assumed uniform $L^{1}$-limit (13.11) implies we may pick $\delta_{o}>0$ small enough to insure that $\delta<\delta_{o}$ implies

$$
\iint_{\mathbb{T}^{D} \times[0, T]}\left|\psi_{\epsilon}^{\delta}\right| \mathrm{d} x \mathrm{~d} t<\frac{\eta}{2 \lambda} \quad \text { for every } \epsilon .
$$

The above two inequalities show that $\delta<\delta_{o}$ implies

$$
\begin{aligned}
\iint_{\mathbb{T}^{D} \times[0, T]}\left|\psi_{\epsilon}^{\delta}\right|^{2} \mathrm{~d} x \mathrm{~d} t \leq & \iint_{\Omega_{\epsilon}^{\delta}(\lambda)}\left|\psi_{\epsilon}^{\delta}\right|^{2} \mathrm{~d} x \mathrm{~d} t \\
& +\lambda \iint_{\mathbb{T}^{D \times[0, T]}}\left|\psi_{\epsilon}^{\delta}\right| \mathrm{d} x \mathrm{~d} t \\
\leq & \frac{\eta}{2}+\lambda \frac{\eta}{2 \lambda}=\eta \quad \text { for every } \epsilon .
\end{aligned}
$$

Because $\eta$ was arbitrary while $\delta_{o}$ was independent of $\epsilon$, the assertion (13.12) follows. 
13.2. Mollified Quadratic Limits. The main result of this section is the following proposition taken from [36] (also see [35, 37]).

Proposition 13.3. For every $\delta>0$ one has

$$
\left.\begin{array}{rl}
\lim _{\epsilon \rightarrow 0} \Pi \nabla_{x} \cdot\left(\tilde{u}_{\epsilon}^{\delta} \otimes \tilde{u}_{\epsilon}^{\delta}\right) & =\Pi \nabla_{x} \cdot\left(u^{\delta} \otimes u^{\delta}\right) \\
\lim _{\epsilon \rightarrow 0} \nabla_{x} \cdot\left(\tilde{\theta}_{\epsilon}^{\delta} \tilde{u}_{\epsilon}^{\delta}\right) & =\nabla_{x} \cdot\left(\theta^{\delta} u^{\delta}\right)
\end{array}\right\} \quad \text { in } w-L_{l o c}^{1}\left(\mathrm{~d} t ; \mathcal{D}^{\prime}\left(\mathbb{T}^{D}\right)\right)
$$

Proof. Introduce the new fluid variables

$$
\begin{array}{ll}
\tilde{w}_{\epsilon}^{\delta}=\Pi \tilde{u}_{\epsilon}^{\delta}, & \tilde{v}_{\epsilon}^{\delta}=\Pi^{\perp} \tilde{u}_{\epsilon}^{\delta}, \\
\tilde{\sigma}_{\epsilon}^{\delta}=\frac{D}{2} \tilde{\theta}_{\epsilon}^{\delta}-\tilde{\rho}_{\epsilon}^{\delta}, & \tilde{\pi}_{\epsilon}^{\delta}=\tilde{\rho}_{\epsilon}^{\delta}+\tilde{\theta}_{\epsilon}^{\delta},
\end{array}
$$

where $\Pi$ is the Leray projection onto divergence-free vector-fields in $L^{2}\left(\mathrm{~d} x ; \mathbb{R}^{D}\right)$. Here $\tilde{w}_{\epsilon}^{\delta}$ and $\tilde{v}_{\epsilon}^{\delta}$ are respectively the divergence-free and gradient components of $\tilde{u}_{\epsilon}^{\delta}$, while $\tilde{\sigma}_{\epsilon}^{\delta}$ and $\tilde{\pi}_{\epsilon}^{\delta}$ are the infinitesimal entropy and pressure fluctuations associated with $\tilde{g}_{\epsilon}^{\delta}$.

Because for every $\zeta \in \operatorname{span}\left\{1, v_{1}, \ldots, v_{D},|v|^{2}\right\}$ one has $\left\langle\zeta \tilde{g}_{\epsilon}\right\rangle \rightarrow\langle\zeta g\rangle$ in $w-L_{l o c}^{2}\left(\mathrm{~d} t ; w-L^{2}(\mathrm{~d} x)\right)$ as $\epsilon \rightarrow 0$, it can be easily shown that for every $s \geq 0$ and $\delta>0$ one has

$$
\lim _{\epsilon \rightarrow 0}\left\langle\zeta \tilde{g}_{\epsilon}^{\delta}\right\rangle=\left\langle\zeta g^{\delta}\right\rangle \quad \text { in } w-L_{l o c}^{2}\left(\mathrm{~d} t ; w-H^{s}(\mathrm{~d} x)\right),
$$

where $H^{s}(\mathrm{~d} x)$ denotes the $s^{\text {th }}$ Sobolev space. In particular, for every $s>0$ and $\delta>0$ the families $\tilde{w}_{\epsilon}^{\delta}, \tilde{v}_{\epsilon}^{\delta}, \tilde{\sigma}_{\epsilon}^{\delta}$, and $\tilde{\pi}_{\epsilon}^{\delta}$ satisfy

$$
\left.\begin{array}{ll}
\lim _{\epsilon \rightarrow 0} \tilde{w}_{\epsilon}^{\delta}=u^{\delta}, & \lim _{\epsilon \rightarrow 0} \tilde{v}_{\epsilon}^{\delta}=0, \\
\lim _{\epsilon \rightarrow 0} \tilde{\sigma}_{\epsilon}^{\delta}=\frac{D+2}{2} \theta^{\delta}, & \lim _{\epsilon \rightarrow 0} \tilde{\pi}_{\epsilon}^{\delta}=0,
\end{array}\right\} \text { in } w-L_{l o c}^{2}\left(\mathrm{~d} t ; w-H^{s}(\mathrm{~d} x)\right),
$$

where $u^{\delta}=j^{\delta} * u$ and $\theta^{\delta}=j^{\delta} * \theta$.

Because $\tilde{u}_{\epsilon}^{\delta}$ and $\tilde{\theta}_{\epsilon}^{\delta}$ decompose as

$$
\tilde{u}_{\epsilon}^{\delta}=\tilde{w}_{\epsilon}^{\delta}+\tilde{v}_{\epsilon}^{\delta}, \quad \frac{D+2}{2} \tilde{\theta}_{\epsilon}^{\delta}=\tilde{\sigma}_{\epsilon}^{\delta}+\tilde{\pi}_{\epsilon}^{\delta},
$$

the quadratic terms $\tilde{u}_{\epsilon}^{\delta} \otimes \tilde{u}_{\epsilon}^{\delta}$ and $\tilde{\theta}_{\epsilon}^{\delta} \tilde{u}_{\epsilon}^{\delta}$ decompose as

$$
\begin{aligned}
\tilde{u}_{\epsilon}^{\delta} \otimes \tilde{u}_{\epsilon}^{\delta} & =\tilde{w}_{\epsilon}^{\delta} \otimes \tilde{w}_{\epsilon}^{\delta}+\tilde{w}_{\epsilon}^{\delta} \otimes \tilde{v}_{\epsilon}^{\delta}+\tilde{v}_{\epsilon}^{\delta} \otimes \tilde{w}_{\epsilon}^{\delta}+\tilde{v}_{\epsilon}^{\delta} \otimes \tilde{v}_{\epsilon}^{\delta}, \\
\frac{D+2}{2} \tilde{\theta}_{\epsilon}^{\delta} \tilde{u}_{\epsilon}^{\delta} & =\tilde{\sigma}_{\epsilon}^{\delta} \tilde{w}_{\epsilon}^{\delta}+\tilde{\sigma}_{\epsilon}^{\delta} \tilde{v}_{\epsilon}^{\delta}+\tilde{\pi}_{\epsilon}^{\delta} \tilde{w}_{\epsilon}^{\delta}+\tilde{\pi}_{\epsilon}^{\delta} \tilde{v}_{\epsilon}^{\delta} .
\end{aligned}
$$

We will consider the limit of each term on the right-hand sides above as $\epsilon \rightarrow 0$. 
It follows from (7.6) that $\tilde{w}_{\epsilon}^{\delta}, \tilde{v}_{\epsilon}^{\delta}, \tilde{\sigma}_{\epsilon}^{\delta}$, and $\tilde{\pi}_{\epsilon}^{\delta}$ satisfy the approximate conservation laws

$$
\begin{array}{rlrl}
\partial_{t} \tilde{w}_{\epsilon}^{\delta} & =\Pi \tilde{J}_{\epsilon}^{\delta}, & \partial_{t} \tilde{v}_{\epsilon}^{\delta}+\frac{1}{\epsilon} \nabla_{x} \tilde{\pi}_{\epsilon}^{\delta}=\Pi^{\perp} \tilde{J}_{\epsilon}^{\delta}, \\
\partial_{t} \tilde{\sigma}_{\epsilon}^{\delta}=\tilde{K}_{\epsilon}^{\delta}, & \partial_{t} \tilde{\pi}_{\epsilon}^{\delta}+\frac{1}{\epsilon} \frac{D+2}{D} \nabla_{x} \cdot \tilde{\pi}_{\epsilon}^{\delta}=\tilde{I}_{\epsilon}^{\delta},
\end{array}
$$

where $\tilde{I}_{\epsilon}^{\delta}, \tilde{J}_{\epsilon}^{\delta}$, and $\tilde{K}_{\epsilon}^{\delta}$ are defined by

$$
\begin{aligned}
\tilde{I}_{\epsilon}^{\delta} & =\frac{1}{\epsilon} j^{\delta} *\left(\left\langle\frac{1}{D}|v|^{2} \Gamma_{\epsilon}^{\prime}\left(G_{\epsilon}\right) q_{\epsilon}\right\rangle-\frac{2}{D} \nabla_{x} \cdot\left\langle B \tilde{g}_{\epsilon}\right\rangle\right), \\
\tilde{J}_{\epsilon}^{\delta} & \left.=\frac{1}{\epsilon} j^{\delta} *\left(\left\langle v \Gamma_{\epsilon}^{\prime}\left(G_{\epsilon}\right) q_{\epsilon}\right\rangle\right\rangle-\nabla_{x} \cdot\left\langle A \tilde{g}_{\epsilon}\right\rangle\right), \\
\tilde{K}_{\epsilon}^{\delta} & =\frac{1}{\epsilon} j^{\delta} *\left(\left\langle\left(\frac{1}{2}|v|^{2}-\frac{D+2}{2}\right) \Gamma_{\epsilon}^{\prime}\left(G_{\epsilon}\right) q_{\epsilon}\right\rangle-\nabla_{x} \cdot\left\langle B \tilde{g}_{\epsilon}\right\rangle\right) .
\end{aligned}
$$

Because $\tilde{J}_{\epsilon}^{\delta}$ and $\tilde{K}_{\epsilon}^{\delta}$ are relatively compact in $w-L_{l o c}^{1}\left(\mathrm{~d} t ; w-H^{s}(\mathrm{~d} x)\right)$, it follows from the first column of (13.19) that the families $\tilde{w}_{\epsilon}^{\delta}$ and $\tilde{\sigma}_{\epsilon}^{\delta}$ are equicontinuous in $C\left([0, \infty) ; w-L^{2}(\mathrm{~d} x)\right)$. Because these $\epsilon$-families are also equibounded in $L^{2}(\mathrm{~d} x)$, the Arzela-Ascoli Theorem implies that they are relatively compact in $C\left([0, \infty) ; w-L^{2}(\mathrm{~d} x)\right)$. Because $(13.16)$ holds for $s=0$, it follows that

(13.21) $\lim _{\epsilon \rightarrow 0} \tilde{w}_{\epsilon}^{\delta}=u^{\delta}, \quad \lim _{\epsilon \rightarrow 0} \tilde{\sigma}_{\epsilon}^{\delta}=\frac{D+2}{2} \theta^{\delta}, \quad$ in $C\left([0, \infty) ; w-L^{2}(\mathrm{~d} x)\right)$.

Because for every $s>0$ one has the continuous embedding

$$
w-L_{l o c}^{2}\left(\mathrm{~d} t ; w-H^{s}(\mathrm{~d} x)\right) \cap C\left([0, \infty) ; w-L^{2}(\mathrm{~d} x)\right) \rightarrow L_{l o c}^{2}\left(\mathrm{~d} t ; L^{2}(\mathrm{~d} x)\right),
$$

the limits (13.16) for $s>0$ and (13.21) imply that the families $\tilde{w}_{\epsilon}^{\delta}$ and $\tilde{\sigma}_{\epsilon}^{\delta}$ satisfy the strong limits

$$
\lim _{\epsilon \rightarrow 0} \tilde{w}_{\epsilon}^{\delta}=u^{\delta}, \quad \lim _{\epsilon \rightarrow 0} \tilde{\sigma}_{\epsilon}^{\delta}=\frac{D+2}{2} \theta^{\delta}, \quad \text { in } L_{l o c}^{2}\left(\mathrm{~d} t ; L^{2}(\mathrm{~d} x)\right) .
$$

When this result is combined with the weak limits for the families $\tilde{v}_{\epsilon}^{\delta}$ and $\tilde{\pi}_{\epsilon}^{\delta}$ found in (13.16), we obtain

$$
\left.\begin{array}{rl}
\lim _{\epsilon \rightarrow 0} \tilde{w}_{\epsilon}^{\delta} \otimes \tilde{w}_{\epsilon}^{\delta} & =u^{\delta} \otimes u^{\delta}, \\
\lim _{\epsilon \rightarrow 0} \tilde{w}_{\epsilon}^{\delta} \otimes \tilde{v}_{\epsilon}^{\delta} & =\lim _{\epsilon \rightarrow 0} \tilde{v}_{\epsilon}^{\delta} \otimes \tilde{w}_{\epsilon}^{\delta}=0, \\
\lim _{\epsilon \rightarrow 0} \tilde{\sigma}_{\epsilon}^{\delta} \tilde{w}_{\epsilon}^{\delta} & =\frac{D+2}{2} \theta^{\delta} u^{\delta}, \\
\lim _{\epsilon \rightarrow 0} \tilde{\sigma}_{\epsilon}^{\delta} \tilde{v}_{\epsilon}^{\delta} & =\lim _{\epsilon \rightarrow 0} \tilde{\pi}_{\epsilon}^{\delta} \tilde{w}_{\epsilon}^{\delta}=0,
\end{array}\right\} \quad \text { in } L_{l o c}^{1}\left(\mathrm{~d} t ; L^{1}(\mathrm{~d} x)\right)
$$

These limits treat all but the last term on the right-hand side of each decomposition in (13.18). 
It follows from the second column of (13.19) that the families $\tilde{v}_{\epsilon}^{\delta}$ and $\tilde{\pi}_{\epsilon}^{\delta}$ satisfy

$$
\begin{aligned}
\nabla_{x} \cdot\left(\tilde{v}_{\epsilon}^{\delta} \otimes \tilde{v}_{\epsilon}^{\delta}\right)= & \frac{1}{2} \nabla_{x}\left|\tilde{v}_{\epsilon}^{\delta}\right|^{2}-\frac{D}{D+2} \nabla_{x}\left(\tilde{\pi}_{\epsilon}^{\delta}\right)^{2}-\epsilon \frac{D}{D+2} \partial_{t}\left(\tilde{\pi}_{\epsilon}^{\delta} \tilde{v}_{\epsilon}^{\delta}\right) \\
& +\epsilon \frac{D}{D+2}\left(\tilde{\pi}_{\epsilon}^{\delta} \Pi^{\perp} \tilde{J}_{\epsilon}^{\delta}+\tilde{v}_{\epsilon}^{\delta} \tilde{I}_{\epsilon}^{\delta}\right), \\
\nabla_{x} \cdot\left(\tilde{\pi}_{\epsilon}^{\delta} \tilde{v}_{\epsilon}^{\delta}\right)= & -\epsilon \partial_{t}\left(\frac{1}{2}\left|\tilde{v}_{\epsilon}^{\delta}\right|^{2}+\frac{1}{2} \frac{D}{D+2}\left(\tilde{\pi}_{\epsilon}^{\delta}\right)^{2}\right) \\
& +\epsilon\left(\tilde{v}_{\epsilon}^{\delta} \Pi^{\perp} \tilde{J}_{\epsilon}^{\delta}+\frac{D}{D+2} \tilde{\pi}_{\epsilon}^{\delta} \tilde{I}_{\epsilon}^{\delta}\right),
\end{aligned}
$$

Because $\Pi \nabla_{x}=0$ and the $\epsilon$-families $\tilde{v}_{\epsilon}^{\delta}$ and $\tilde{\pi}_{\epsilon}^{\delta}$ are bounded in $L^{\infty}\left(\mathrm{d} t ; L^{2}(\mathrm{~d} x)\right) \cap$ $C\left([0, \infty) ; H^{s}(\mathrm{~d} x)\right)$, while the $\epsilon$-families $\tilde{I}_{\epsilon}^{\delta}$ and $\tilde{J}_{\epsilon}^{\delta}$ are bounded in $L_{l o c}^{1}\left(\mathrm{~d} t ; L^{2}(\mathrm{~d} x)\right)$, it follows from the above relations that

$$
\left.\begin{array}{rl}
\lim _{\epsilon \rightarrow 0} \Pi \nabla_{x} \cdot\left(\tilde{v}_{\epsilon}^{\delta} \otimes \tilde{v}_{\epsilon}^{\delta}\right) & =0 \\
\lim _{\epsilon \rightarrow 0} \nabla_{x} \cdot\left(\tilde{\pi}_{\epsilon}^{\delta} \tilde{v}_{\epsilon}^{\delta}\right) & =0
\end{array}\right\} \quad \text { in } w-L_{l o c}^{1}\left(\mathrm{~d} t ; \mathcal{D}^{\prime}\left(\mathbb{T}^{D}\right)\right)
$$

These limits treat the last term on the right-hand side of each decomposition in (13.18). Assertion (13.13) of the proposition follows by using decomposition (13.18) along with the limits in (13.23) and (13.25). 
Acknowledgments. C.D.L. was partially supported by the NSF under grant DMS-9803753. N.M. was partially supported by the NSF under grant DMS-0703145. The authors would like to thank F. Golse and L. Saint-Raymond for many discussions about the hydrodynamic limit. N. M would like to thank D. Arsenio for a careful reading of the paper.

\section{REFERENCES}

[1] C. Bardos, F. Golse, and D. Levermore, Sur les limites asymptotiques de la théorie cinétique conduisant à la dynamique des fluides incompressibles, C.R. Acad. Sci. Paris Sr. I Math. 309 (1989), 727-732.

[2] C. Bardos, F. Golse, and D. Levermore, Fluid Dynamic Limits of Kinetic Equations I: Formal Derivations, J. Stat. Phys. 63 (1991), 323-344.

[3] C. Bardos, F. Golse, and C.D. Levermore, Fluid Dynamic Limits of Kinetic Equations II: Convergence Proofs for the Boltzmann Equation, Commun. Pure \& Appl. Math 46 (1993), 667-753.

[4] C. Bardos, F. Golse, and C.D. Levermore, Acoustic and Stokes Limits for the Boltzmann Equation, C.R. Acad. Sci. Paris 327 (1999), 323-328.

[5] C. Bardos, F. Golse, and C.D. Levermore, The Acoustic Limit for the Boltzmann Equation, Archive Rat. Mech. \& Anal. 153 (2000), no. 3, 177-204.

[6] C. Bardos and C.D. Levermore, Kinetic Equations and an Incompressible Fluid Dynamical Limit that Recovers Viscous Heating, (in preparation 2003).

[7] C. Bardos and S. Ukai, The Classical Incompressible Navier-Stokes Limit of the Boltzmann Equation, Math. Models \& Meth Appl. Sci. 1 (1991), 235-257.

[8] L. Boltzmann, Weitere Studien über das Wärmegleichgewicht unter Gasmolekülen, Sitzungs. Akad. Wiss. Wein 66 (1872), 275-370; English: Further Studies on the Thermal Equilibrium of Gas Molecules, in Kinetic Theory 2, S.G. Brush (ed.), Pergamon Press, London, 1966, 88-174.

[9] R. Caflisch, The Boltzmann Equation with a Soft Potential I: Linear, Spatially Homogeneous, Commun. Math. Phys. 74 (1980), 71-95.

[10] R. Caflisch, The Fluid Dynamical Limit of the Nonlinear Boltzmann Equation, Commun. Pure \& Appl. Math. 33 (1980), 651-666.

[11] C. Cercignani, The Boltzmann Equation and its Applications, Springer-Verlag, New York, 1988.

[12] C. Cercignani, R. Illner, and M. Pulvirenti, The Mathematical Theory of Dilute Gases, Appl. Math. Sci. 106, Springer-Verlag, New York, 1994.

[13] P. Constantin and C. Foias, Navier-Stokes Equations, Chicago Lectures in Mathematics, The University of Chicago Press, Chicago, 1988.

[14] A. DeMasi, R. Esposito, and J. Lebowitz, Incompressible Navier-Stokes and Euler Limits of the Boltzmann Equation, Commun. Pure \& Appl. Math. 42 (1990), 1189-1214.

[15] R.J. DiPerna and P.-L. Lions, On the Cauchy Problem for the Boltzmann Equation: Global Existence and Weak Stability Results, Annals of Math. 130 (1990), 321-366.

[16] D. Enskog, Kinetische Theorie der Vorgänge in mässig verdünnten Gasen, I. Allgemeiner Teil, Almqvist \& Wiksell, Uppsala, 1917; English: Kinetic Theory of Processes in Dilute Gases, in Kinetic Theory 3; S.G. Brush ed., Pergamon Press, Oxford, 1972, 125-225. 
[17] R. Glassey, The Cauchy Problem in Kinetic Theory, Society for Industrial and Applied Mathematics (SIAM), Philadelphia, 1996.

[18] F. Golse, From Kinetic to Macroscopic Models, in Kinetic Equations and Asymptotic Theory, B. Perthame and L. Desvillettes eds., Series in Applied Mathematics 4, Gauthier-Villars, Paris, 2000, 41-126.

[19] F. Golse and C.D. Levermore, Stokes-Fourier and Acoustic Limits for the Boltsmann Equation: Convergence Proofs, Commun. Pure \& Appl. Math. 55 (2002), no. 3, 336-393.

[20] F. Golse, P.-L. Lions, B. Perthame, R. Sentis, Regularity of the Moments of the Solution of a Transport Equation, J. Funct. Anal. 76 (1988), 110-125.

[21] F. Golse, B. Perthame, R. Sentis, Un résultat de compacité pour les équations de transport et application au calcul de la limite de la valeur propre principale de l'opérateur de transport, C.R. Acad. Sci. Paris Sr. I Math. 301 (1985), 341-344.

[22] F. Golse and F. Poupaud, Un rsultat de compacit pour l'quation de Boltzmann avec potentiel mou. Application au problme de demi-espace, C. R. Acad. Sci. Paris Sr. I Math. 303 (1986), 583-586.

[23] F. Golse and L. Saint-Raymond, The Navier-Stokes Limit for the Boltzmann Equation, C. R. Acad. Sci. Paris Sr. I Math. 333 (2001), 897-902.

[24] F. Golse and L. Saint-Raymond, Velocity Averaging in $L^{1}$ for the Transport Equation, C. R. Acad. Sci. Paris Sr. I Math. 334 (2002), 557-562.

[25] F. Golse and L. Saint-Raymond, The Navier-Stokes Limit of the Boltzmann Equation for Bounded Collision Kernels, Invent. Math. 155 (2004), 81-161.

[26] F. Golse and L. Saint-Raymond, The Navier-Stokes Limit of the Boltzmann Equation for Hard Sphere Collision Kernels, (talk, April 2004).

[27] H. Grad, Principles of the Kinetic Theory of Gases, in Handbuch der Physik 12, S. Flügge ed., Springer-Verlag, Berlin, 1958, 205-294.

[28] D. Hilbert, Begründung der kinetischen Gastheorie, Math. Annalen 72 (1912), 562-577; English: Foundations of the Kinetic Theory of Gases, in Kinetic Theory 3; S.G. Brush (ed.), Pergamon Press, Oxford, 1972, 89-101.

[29] J. Leray, Sur le mouvement d'un fluide visqueux emplissant l'espace, Acta Math. 63 (1934), 193-248.

[30] C.D. Levermore, Entropic Convergence and the Linearized Limit for the Boltzmann Equation, Commun. P.D.E. 18 (1993), 1231-1248.

[31] P.-L. Lions, Compactness in Boltzmann's Equation via Fourier Integral Operators and Applications, I, II, \& III. J. Math. Kyoto Univ. 34 (1994), 391-427, 429-461, 539-584.

[32] P.-L. Lions, Mathematical Topics in Fluid Mechanics, Vol. 1: Incompressible Models, Oxford Lecture Series in Mathematics and its Applications 3. Oxford Science Publications, The Clarendon Press, Oxford University Press, New York, 1996.

[33] P.-L. Lions, Mathematical Topics in Fluid Mechanics, Vol. 2: Compressible Models, Oxford Lecture Series in Mathematics and its Applications 3. Oxford Science Publications, The Clarendon Press, Oxford University Press, New York, 1998.

[34] P.-L. Lions and N. Masmoudi, Incompressible limit for a viscous compressible fluid, J. Math. Pures Appl. 77 (1998), 585-627. 
[35] P.-L. Lions and N. Masmoudi, Une approche locale de la limite incompressible. C. R. Acad. Sci. Paris Sr. I Math. 329 (1999), 387-392.

[36] P.-L. Lions and N. Masmoudi, From the Boltzmann Equations to the Equations of Incompressible Fluid Mechanics, I, Archive Rat. Mech. \& Anal. 158 (2001), 173-193.

[37] P.-L. Lions and N. Masmoudi, From the Boltzmann Equations to the Equations of Incompressible Fluid Mechanics, II, Archive Rat. Mech. \& Anal. 158 (2001), $195-211$.

[38] N. Masmoudi. Examples of singular limits in hydrodynamics. In Evolutionary equations. Vol. III, Handb. Differ. Equ., pages 195-276. Elsevier/NorthHolland, Amsterdam, 2007.

[39] J.C. Maxwell, On the Dynamical Theory of Gases, Philos. Trans. Roy. Soc. London Ser. A 157 (1866), 49-88; Reprinted in The Scientific Letters and Papers of James Clerk Maxwell, Vol. 2, Dover, New York, 1965, 26-78.

[40] Saint-Raymond, Discrete-Time Navier-Stokes Limit for the BGK Boltzmann Equation, Commun. P.D.E 27 (2002), 149-184.

[41] Saint-Raymond, Du modèle BGK de l'équation de Boltzmann aux équations d' Euler des fluides incompressibles, Bull. Sci. Math. 126 (2002), 493-506.

[42] Saint-Raymond, From the Boltzmann BGK Equation to the Navier-Stokes System, Ann. Scient. École Norm. Sup. 36 (2003), 271-317.

[43] W. Sun, Fredholm Alternatives for Linearized Boltzmann Collision Operators with Weakly Cutoff Kernels, (preprint 2005).

[44] C. Villani, Limites hydrodynamiques de l'équation de Boltzmann [d'après C. Bardos, F. Golse, D. Levermore, P.-L. Lions, N. Masmoudi, L. SaintRaymond], Séminaire Bourbaki 2000-2001, exposition 893.

(C.D. Levermore) Department of Mathematics, and Institute for Physical Science and Technology, University of Maryland, College Park, MD 20742-4015, USA

E-mail address: lvrmr@math.umd.edu

(N. Masmoudi) Courant Institute of Mathematical Sciences, New York University, 251 Mercer Street, New York, NY 10012, USA

E-mail address: masmoudi@cims.nyu.edu 